\title{
An Introduction to Hyperplane Arrangements
}

\author{
Richard P. Stanley
}





\section{Contents}

An Introduction to Hyperplane Arrangements 1

Lecture 1. Basic definitions, the intersection poset and the characteristic polynomial

Exercises

Lecture 2. Properties of the intersection poset and graphical arrangements Exercises

Lecture 3. Matroids and geometric lattices

Lecture 4. Broken circuits, modular elements, and supersolvability

Lecture 5. Finite fields

Lecture 6. Separating Hyperplanes $\quad 89$

Exercises

Bibliography

109 



\title{
An Introduction to Hyperplane Arrangements
}

\author{
Richard P. Stanley ${ }^{1,2}$
}

$1_{\text {version of February } 26,2006}$

2 The author was supported in part by NSF grant DMS-9988459. He is grateful to Lauren Williams for her careful reading of the original manuscript and many helpful suggestions, and to Hélène Barcelo and Guangfeng Jiang for a number of of additional suggestions. Students in 18.315, taught at MIT during fall 2004, also made some helpful contributions. 


\section{LECTURE 1 \\ Basic definitions, the intersection poset and the characteristic polynomial}

\subsection{Basic definitions}

The following notation is used throughout for certain sets of numbers:

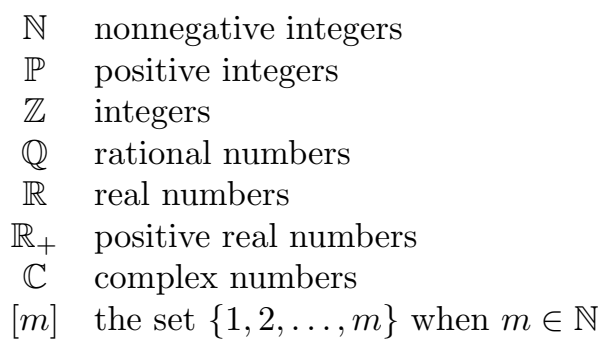

We also write $\left[t^{k}\right] \chi(t)$ for the coefficient of $t^{k}$ in the polynomial or power series $\chi(t)$. For instance, $\left[t^{2}\right](1+t)^{4}=6$.

A finite hyperplane arrangement $\mathcal{A}$ is a finite set of affine hyperplanes in some vector space $V \cong K^{n}$, where $K$ is a field. We will not consider infinite hyperplane arrangements or arrangements of general subspaces or other objects (though they have many interesting properties), so we will simply use the term arrangement for a finite hyperplane arrangement. Most often we will take $K=\mathbb{R}$, but as we will see even if we're only interested in this case it is useful to consider other fields as well. To make sure that the definition of a hyperplane arrangement is clear, we define a linear hyperplane to be an $(n-1)$-dimensional subspace $H$ of $V$, i.e.,

$$
H=\{v \in V: \alpha \cdot v=0\},
$$

where $\alpha$ is a fixed nonzero vector in $V$ and $\alpha \cdot v$ is the usual dot product:

$$
\left(\alpha_{1}, \ldots, \alpha_{n}\right) \cdot\left(v_{1}, \ldots, v_{n}\right)=\sum \alpha_{i} v_{i} .
$$

An affine hyperplane is a translate $J$ of a linear hyperplane, i.e.,

$$
J=\{v \in V: \alpha \cdot v=a\},
$$

where $\alpha$ is a fixed nonzero vector in $V$ and $a \in K$.

If the equations of the hyperplanes of $\mathcal{A}$ are given by $L_{1}(x)=a_{1}, \ldots, L_{m}(x)=$ $a_{m}$, where $x=\left(x_{1}, \ldots, x_{n}\right)$ and each $L_{i}(x)$ is a homogeneous linear form, then we call the polynomial

$$
Q_{\mathcal{A}}(x)=\left(L_{1}(x)-a_{1}\right) \cdots\left(L_{m}(x)-a_{m}\right)
$$

the defining polynomial of $\mathcal{A}$. It is often convenient to specify an arrangement by its defining polynomial. For instance, the arrangement $\mathcal{A}$ consisting of the $n$ coordinate hyperplanes has $Q_{\mathcal{A}}(x)=x_{1} x_{2} \cdots x_{n}$.

Let $\mathcal{A}$ be an arrangement in the vector space $V$. The dimension $\operatorname{dim}(\mathcal{A})$ of $\mathcal{A}$ is defined to be $\operatorname{dim}(V)(=n)$, while the $\operatorname{rank} \operatorname{rank}(\mathcal{A})$ of $\mathcal{A}$ is the dimension of the space spanned by the normals to the hyperplanes in $\mathcal{A}$. We say that $\mathcal{A}$ is essential if $\operatorname{rank}(\mathcal{A})=\operatorname{dim}(\mathcal{A})$. Suppose that $\operatorname{rank}(\mathcal{A})=r$, and take $V=K^{n}$. Let 
$Y$ be a complementary space in $K^{n}$ to the subspace $X$ spanned by the normals to hyperplanes in $\mathcal{A}$. Define

$$
W=\{v \in V: v \cdot y=0 \forall y \in Y\} .
$$

If $\operatorname{char}(K)=0$ then we can simply take $W=X$. By elementary linear algebra we have

$$
\operatorname{codim}_{W}(H \cap W)=1
$$

for all $H \in \mathcal{A}$. In other words, $H \cap W$ is a hyperplane of $W$, so the set $\mathcal{A}_{W}:=$ $\{H \cap W: H \in \mathcal{A}\}$ is an essential arrangement in $W$. Moreover, the arrangements $\mathcal{A}$ and $\mathcal{A}_{W}$ are "essentially the same," meaning in particular that they have the same intersection poset (as defined in Definition 1.1). Let us call $\mathcal{A}_{W}$ the essentialization of $\mathcal{A}$, denoted $\operatorname{ess}(\mathcal{A})$. When $K=\mathbb{R}$ and we take $W=X$, then the arrangement $\mathcal{A}$ is obtained from $\mathcal{A}_{W}$ by "stretching" the hyperplane $H \cap W \in \mathcal{A}_{W}$ orthogonally to $W$. Thus if $W^{\perp}$ denotes the orthogonal complement to $W$ in $V$, then $H^{\prime} \in \mathcal{A}_{W}$ if and only if $H^{\prime} \oplus W^{\perp} \in \mathcal{A}$. Note that in characteristic $p$ this type of reasoning fails since the orthogonal complement of a subspace $W$ can intersect $W$ in a subspace of dimension greater than 0 .

Example 1.1. Let $\mathcal{A}$ consist of the lines $x=a_{1}, \ldots, x=a_{k}$ in $K^{2}$ (with coordinates $x$ and $y)$. Then we can take $W$ to be the $x$-axis, and $\operatorname{ess}(\mathcal{A})$ consists of the points $x=a_{1}, \ldots, x=a_{k}$ in $K$.

Now let $K=\mathbb{R}$. A region of an arrangement $\mathcal{A}$ is a connected component of the complement $X$ of the hyperplanes:

$$
X=\mathbb{R}^{n}-\bigcup_{H \in \mathcal{A}} H
$$

Let $\mathcal{R}(\mathcal{A})$ denote the set of regions of $\mathcal{A}$, and let

$$
r(\mathcal{A})=\# \mathcal{R}(\mathcal{A})
$$

the number of regions. For instance, the arrangement $\mathcal{A}$ shown below has $r(\mathcal{A})=14$.

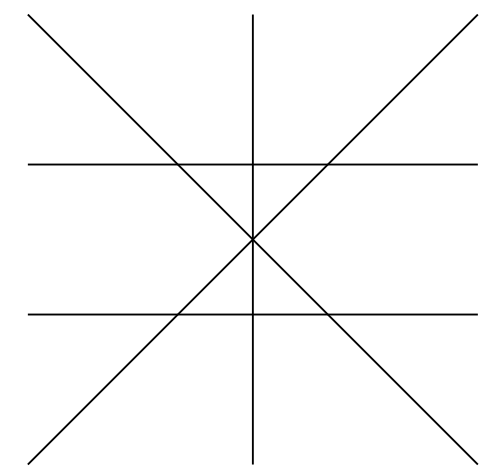

It is a simple exercise to show that every region $R \in \mathcal{R}(\mathcal{A})$ is open and convex (continuing to assume $K=\mathbb{R}$ ), and hence homeomorphic to the interior of an $n$ dimensional ball $\mathbb{B}^{n}$ (Exercise 1). Note that if $W$ is the subspace of $V$ spanned by the normals to the hyperplanes in $\mathcal{A}$, then $R \in \mathcal{R}(\mathcal{A})$ if and only if $R \cap W \in \mathcal{R}\left(\mathcal{A}_{W}\right)$. We say that a region $R \in \mathcal{R}(\mathcal{A})$ is relatively bounded if $R \cap W$ is bounded. If $\mathcal{A}$ is essential, then relatively bounded is the same as bounded. We write $b(\mathcal{A})$ for 
the number of relatively bounded regions of $\mathcal{A}$. For instance, in Example 1.1 take $K=\mathbb{R}$ and $a_{1}<a_{2}<\cdots<a_{k}$. Then the relatively bounded regions are the regions $a_{i}<x<a_{i+1}, 1 \leq i \leq k-1$. In $\operatorname{ess}(\mathcal{A})$ they become the (bounded) open intervals $\left(a_{i}, a_{i+1}\right)$. There are also two regions of $\mathcal{A}$ that are not relatively bounded, viz., $x<a_{1}$ and $x>a_{k}$.

A (closed) half-space is a set $\left\{x \in \mathbb{R}^{n}: x \cdot \alpha \geq c\right\}$ for some $\alpha \in \mathbb{R}^{n}, c \in \mathbb{R}$. If $H$ is a hyperplane in $\mathbb{R}^{n}$, then the complement $\mathbb{R}^{n}-H$ has two (open) components whose closures are half-spaces. It follows that the closure $\bar{R}$ of a region $R$ of $\mathcal{A}$ is a finite intersection of half-spaces, i.e., a (convex) polyhedron (of dimension $n$ ). A bounded polyhedron is called a (convex) polytope. Thus if $R$ (or $\bar{R}$ ) is bounded, then $\bar{R}$ is a polytope (of dimension $n$ ).

An arrangement $\mathcal{A}$ is in general position if

$$
\begin{aligned}
& \left\{H_{1}, \ldots, H_{p}\right\} \subseteq \mathcal{A}, p \leq n \Rightarrow \operatorname{dim}\left(H_{1} \cap \cdots \cap H_{p}\right)=n-p \\
& \left\{H_{1}, \ldots, H_{p}\right\} \subseteq \mathcal{A}, p>n \Rightarrow H_{1} \cap \cdots \cap H_{p}=\emptyset .
\end{aligned}
$$

For instance, if $n=2$ then a set of lines is in general position if no two are parallel and no three meet at a point.

Let us consider some interesting examples of arrangements that will anticipate some later material.

Example 1.2. Let $\mathcal{A}_{m}$ consist of $m$ lines in general position in $\mathbb{R}^{2}$. We can compute $r\left(\mathcal{A}_{m}\right)$ using the sweep hyperplane method. Add a $L$ line to $\mathcal{A}_{k}$ (with $\mathcal{A}_{K} \cup\{L\}$ in general position). When we travel along $L$ from one end (at infinity) to the other, every time we intersect a line in $\mathcal{A}_{k}$ we create a new region, and we create one new region at the end. Before we add any lines we have one region (all of $\mathbb{R}^{2}$ ). Hence

$$
\begin{aligned}
r\left(\mathcal{A}_{m}\right) & =\# \text { intersections }+\# \text { lines }+1 \\
& =\left(\begin{array}{c}
m \\
2
\end{array}\right)+m+1 .
\end{aligned}
$$

Example 1.3. The braid arrangement $\mathcal{B}_{n}$ in $K^{n}$ consists of the hyperplanes

$$
\mathcal{B}_{n}: \quad x_{i}-x_{j}=0, \quad 1 \leq i<j \leq n .
$$

Thus $\mathcal{B}_{n}$ has $\left(\begin{array}{l}n \\ 2\end{array}\right)$ hyperplanes. To count the number of regions when $K=\mathbb{R}$, note that specifying which side of the hyperplane $x_{i}-x_{j}=0$ a point $\left(a_{1}, \ldots, a_{n}\right)$ lies on is equivalent to specifying whether $a_{i}<a_{j}$ or $a_{i}>a_{j}$. Hence the number of regions is the number of ways that we can specify whether $a_{i}<a_{j}$ or $a_{i}>a_{j}$ for $1 \leq i<j \leq n$. Such a specification is given by imposing a linear order on the $a_{i}$ 's. In other words, for each permutation $w \in \mathfrak{S}_{n}$ (the symmetric group of all permutations of $1,2, \ldots, n)$, there corresponds a region $R_{w}$ of $\mathcal{B}_{n}$ given by

$$
R_{w}=\left\{\left(a_{1}, \ldots, a_{n}\right) \in \mathbb{R}^{n}: a_{w(1)}>a_{w(2)}>\cdots>a_{w(n)}\right\} .
$$

Hence $r\left(\mathcal{B}_{n}\right)=n$ !. Rarely is it so easy to compute the number of regions!

Note that the braid arrangement $\mathcal{B}_{n}$ is not essential; indeed, $\operatorname{rank}\left(\mathcal{B}_{n}\right)=n-1$. When $\operatorname{char}(K)$ does not divide $n$ the space $W \subseteq K^{n}$ of equation (1) can be taken to be

$$
W=\left\{\left(a_{1}, \ldots, a_{n}\right) \in K^{n}: a_{1}+\cdots+a_{n}=0\right\} .
$$

The braid arrangement has a number of "deformations" of considerable interest. We will just define some of them now and discuss them further later. All these 
arrangements lie in $K^{n}$, and in all of them we take $1 \leq i<j \leq n$. The reader who likes a challenge can try to compute their number of regions when $K=\mathbb{R}$. (Some are much easier than others.)

- generic braid arrangement: $x_{i}-x_{j}=a_{i j}$, where the $a_{i j}$ 's are "generic" (e.g., linearly independent over the prime field, so $K$ has to be "sufficiently large"). The precise definition of "generic" will be given later. (The prime field of $K$ is its smallest subfield, isomorphic to either $\mathbb{Q}$ or $\mathbb{Z} / p \mathbb{Z}$ for some prime $p$.)

- semigeneric braid arrangement: $x_{i}-x_{j}=a_{i}$, where the $a_{i}$ 's are "generic."

- Shi arrangement: $x_{i}-x_{j}=0,1$ (so $n(n-1)$ hyperplanes in all).

- Linial arrangement: $x_{i}-x_{j}=1$.

- Catalan arrangement: $x_{i}-x_{j}=-1,0,1$.

- semiorder arrangement: $x_{i}-x_{j}=-1,1$.

- threshold arrangement: $x_{i}+x_{j}=0$ (not really a deformation of the braid arrangement, but closely related).

An arrangement $\mathcal{A}$ is central if $\bigcap_{H \in \mathcal{A}} H \neq \emptyset$. Equivalently, $\mathcal{A}$ is a translate of a linear arrangement (an arrangement of linear hyperplanes, i.e., hyperplanes passing through the origin). Many other writers call an arrangement central, rather than linear, if $0 \in \bigcap_{H \in \mathcal{A}} H$. If $\mathcal{A}$ is central with $X=\bigcap_{H \in \mathcal{A}} H$, then $\operatorname{rank}(\mathcal{A})=$ $\operatorname{codim}(X)$. If $\mathcal{A}$ is central, then note also that $b(\mathcal{A})=0$ [why?].

There are two useful arrangements closely related to a given arrangement $\mathcal{A}$. If $\mathcal{A}$ is a linear arrangement in $K^{n}$, then projectivize $\mathcal{A}$ by choosing some $H \in \mathcal{A}$ to be the hyperplane at infinity in projective space $P_{K}^{n-1}$. Thus if we regard

$$
P_{K}^{n-1}=\left\{\left(x_{1}, \ldots, x_{n}\right): x_{i} \in K, \text { not all } x_{i}=0\right\} / \sim,
$$

where $u \sim v$ if $u=\alpha v$ for some $0 \neq \alpha \in K$, then

$$
H=\left(\left\{\left(x_{1}, \ldots, x_{n-1}, 0\right): x_{i} \in K \text {, not all } x_{i}=0\right\} / \sim\right) \cong P_{K}^{n-2} .
$$

The remaining hyperplanes in $\mathcal{A}$ then correspond to "finite" (i.e., not at infinity) projective hyperplanes in $P_{K}^{n-1}$. This gives an arrangement $\operatorname{proj}(\mathcal{A})$ of hyperplanes in $P_{K}^{n-1}$. When $K=\mathbb{R}$, the two regions $R$ and $-R$ of $\mathcal{A}$ become identified in $\operatorname{proj}(\mathcal{A})$. Hence $r(\operatorname{proj}(\mathcal{A}))=\frac{1}{2} r(\mathcal{A})$. When $n=3$, we can draw $P_{\mathbb{R}}^{2}$ as a disk with antipodal boundary points identified. The circumference of the disk represents the hyperplane at infinity. This provides a good way to visualize three-dimensional real linear arrangements. For instance, if $\mathcal{A}$ consists of the three coordinate hyperplanes $x_{1}=0, x_{2}=0$, and $x_{3}=0$, then a projective drawing is given by

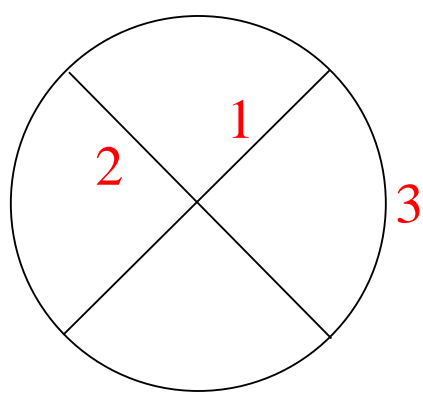


The line labelled $i$ is the projectivization of the hyperplane $x_{i}=0$. The hyperplane at infinity is $x_{3}=0$. There are four regions, so $r(\mathcal{A})=8$. To draw the incidences among all eight regions of $\mathcal{A}$, simply "reflect" the interior of the disk to the exterior:

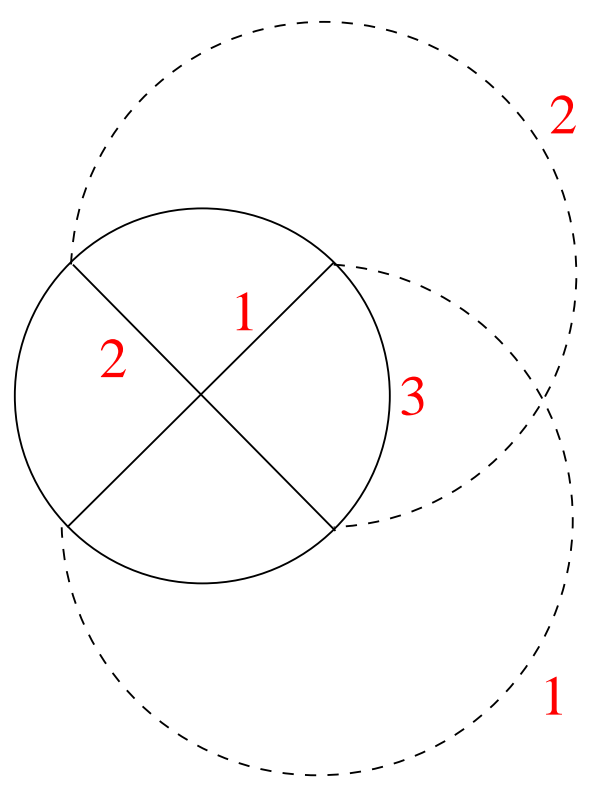

Regarding this diagram as a planar graph, the dual graph is the 3-cube (i.e., the vertices and edges of a three-dimensional cube) [why?].

For a more complicated example of projectivization, Figure $1 \operatorname{shows} \operatorname{proj}\left(\mathcal{B}_{4}\right)$ (where we regard $\mathcal{B}_{4}$ as a three-dimensional arrangement contained in the hyperplane $x_{1}+x_{2}+x_{3}+x_{4}=0$ of $\mathbb{R}^{4}$ ), with the hyperplane $x_{i}=x_{j}$ labelled $i j$, and with $x_{1}=x_{4}$ as the hyperplane at infinity.

We now define an operation which is "inverse" to projectivization. Let $\mathcal{A}$ be an (affine) arrangement in $K^{n}$, given by the equations

$$
L_{1}(x)=a_{1}, \ldots, \quad L_{m}(x)=a_{m}
$$

Introduce a new coordinate $y$, and define a central arrangement $c \mathcal{A}$ (the cone over $\mathcal{A})$ in $K^{n} \times K=K^{n+1}$ by the equations

$$
L_{1}(x)=a_{1} y, \quad \ldots, \quad L_{m}(x)=a_{m} y, \quad y=0 .
$$

For instance, let $\mathcal{A}$ be the arrangement in $\mathbb{R}^{1}$ given by $x=-1, x=2$, and $x=3$. The following figure should explain why $c \mathcal{A}$ is called a cone. 


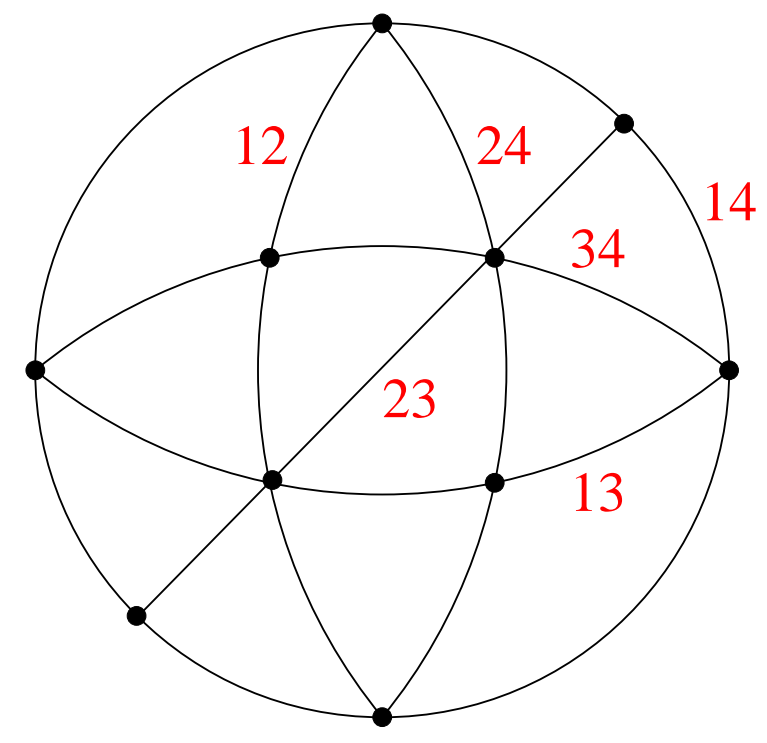

Figure 1. A projectivization of the braid arrangement $\mathcal{B}_{4}$

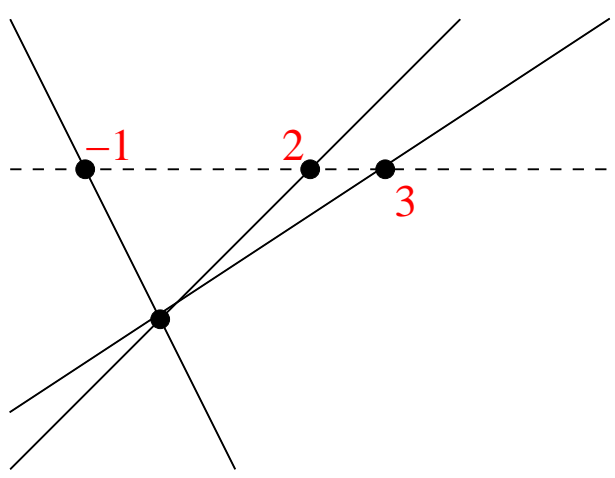

It is easy to see that when $K=\mathbb{R}$, we have $r(c \mathcal{A})=2 r(\mathcal{A})$. In general, $c \mathcal{A}$ has the "same combinatorics as $\mathcal{A}$, times 2." See Exercise 2.1.

\subsection{The intersection poset}

Recall that a poset (short for partially ordered set) is a set $P$ and a relation $\leq$ satisfying the following axioms (for all $x, y, z \in P$ ):

(P1) (reflexivity) $x \leq x$

(P2) (antisymmetry) If $x \leq y$ and $y \leq x$, then $x=y$.

(P3) (transitivity) If $x \leq y$ and $y \leq z$, then $x \leq z$.

Obvious notation such as $x<y$ for $x \leq y$ and $x \neq y$, and $y \geq x$ for $x \leq y$ will be used throughout. If $x \leq y$ in $P$, then the (closed) interval $[x, y]$ is defined by

$$
[x, y]=\{z \in P: x \leq z \leq y\} .
$$

Note that the empty set $\emptyset$ is not a closed interval. For basic information on posets not covered here, see $[\mathbf{3 1}]$. 

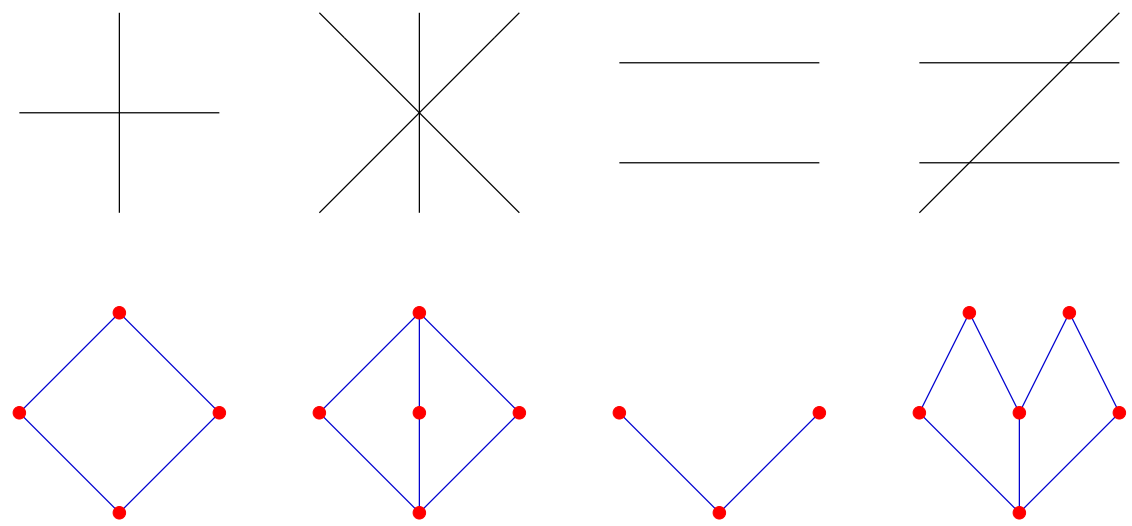

Figure 2. Examples of intersection posets

Definition 1.1. Let $\mathcal{A}$ be an arrangement in $V$, and let $L(\mathcal{A})$ be the set of all nonempty intersections of hyperplanes in $\mathcal{A}$, including $V$ itself as the intersection over the empty set. Define $x \leq y$ in $L(\mathcal{A})$ if $x \supseteq y$ (as subsets of $V$ ). In other words, $L(\mathcal{A})$ is partially ordered by reverse inclusion. We call $L(\mathcal{A})$ the intersection poset of $\mathcal{A}$.

Note. The primary reason for ordering intersections by reverse inclusion rather than ordinary inclusion is Proposition 3.8. We don't want to alter the well-established definition of a geometric lattice or to refer constantly to "dual geometric lattices."

The element $V \in L(\mathcal{A})$ satisfies $x \geq V$ for all $x \in L(\mathcal{A})$. In general, if $P$ is a poset then we denote by $\hat{0}$ an element (necessarily unique) such that $x \geq \hat{0}$ for all $x \in P$. We say that $y$ covers $x$ in a poset $P$, denoted $x \lessdot y$, if $x<y$ and no $z \in P$ satisfies $x<z<y$. Every finite poset is determined by its cover relations. The (Hasse) diagram of a finite poset is obtained by drawing the elements of $P$ as dots, with $x$ drawn lower than $y$ if $x<y$, and with an edge between $x$ and $y$ if $x \lessdot y$. Figure 2 illustrates four arrangements $\mathcal{A}$ in $\mathbb{R}^{2}$, with (the diagram of) $L(\mathcal{A})$ drawn below $\mathcal{A}$.

A chain of length $k$ in a poset $P$ is a set $x_{0}<x_{1}<\cdots<x_{k}$ of elements of $P$. The chain is saturated if $x_{0} \lessdot x_{1} \lessdot \cdots \lessdot x_{k}$. We say that $P$ is graded of rank $n$ if every maximal chain of $P$ has length $n$. In this case $P$ has a rank function rk $: P \rightarrow \mathbb{N}$ defined by:

- $\operatorname{rk}(x)=0$ if $x$ is a minimal element of $P$.

- $\operatorname{rk}(y)=\operatorname{rk}(x)+1$ if $x \lessdot y$ in $P$.

If $x<y$ in a graded poset $P$ then we write $\operatorname{rk}(x, y)=\operatorname{rk}(y)-\operatorname{rk}(x)$, the length of the interval $[x, y]$. Note that we use the notation $\operatorname{rank}(\mathcal{A})$ for the $\operatorname{rank}$ of an arrangement $\mathcal{A}$ but rk for the rank function of a graded poset.

Proposition 1.1. Let $\mathcal{A}$ be an arrangement in a vector space $V \cong K^{n}$. Then the intersection poset $L(\mathcal{A})$ is graded of rank equal to $\operatorname{rank}(\mathcal{A})$. The rank function of $L(\mathcal{A})$ is given by

$$
\operatorname{rk}(x)=\operatorname{codim}(x)=n-\operatorname{dim}(x),
$$

where $\operatorname{dim}(x)$ is the dimension of $x$ as an affine subspace of $V$. 


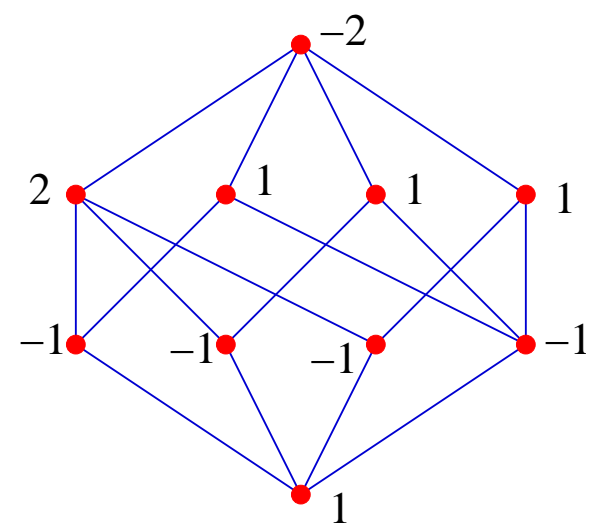

Figure 3. An intersection poset and Möbius function values

Proof. Since $L(\mathcal{A})$ has a unique minimal element $\hat{0}=V$, it suffices to show that (a) if $x \lessdot y$ in $L(\mathcal{A})$ then $\operatorname{dim}(x)-\operatorname{dim}(y)=1$, and (b) all maximal elements of $L(\mathcal{A})$ have dimension $n-\operatorname{rank}(\mathcal{A})$. By linear algebra, if $H$ is a hyperplane and $x$ an affine subspace, then $H \cap x=x$ or $\operatorname{dim}(x)-\operatorname{dim}(H \cap x)=1$, so (a) follows. Now suppose that $x$ has the largest codimension of any element of $L(\mathcal{A})$, say $\operatorname{codim}(x)=d$. Thus $x$ is an intersection of $d$ linearly independent hyperplanes (i.e., their normals are linearly independent) $H_{1}, \ldots, H_{d}$ in $\mathcal{A}$. Let $y \in L(\mathcal{A})$ with $e=\operatorname{codim}(y)<d$. Thus $y$ is an intersection of $e$ hyperplanes, so some $H_{i}(1 \leq i \leq d)$ is linearly independent from them. Then $y \cap H_{i} \neq \emptyset$ and $\operatorname{codim}\left(y \cap H_{i}\right)>\operatorname{codim}(y)$. Hence $y$ is not a maximal element of $L(\mathcal{A})$, proving (b).

\subsection{The characteristic polynomial}

A poset $P$ is locally finite if every interval $[x, y]$ is finite. Let $\operatorname{Int}(P)$ denote the set of all closed intervals of $P$. For a function $f: \operatorname{Int}(P) \rightarrow \mathbb{Z}$, write $f(x, y)$ for $f([x, y])$. We now come to a fundamental invariant of locally finite posets.

Definition 1.2. Let $P$ be a locally finite poset. Define a function $\mu=\mu_{P}$ : $\operatorname{Int}(P) \rightarrow \mathbb{Z}$, called the Möbius function of $P$, by the conditions:

$$
\begin{aligned}
& \mu(x, x)=1, \text { for all } x \in P \\
& \mu(x, y)=-\sum_{x \leq z<y} \mu(x, z), \text { for all } x<y \text { in } P .
\end{aligned}
$$

This second condition can also be written

$$
\sum_{x \leq z \leq y} \mu(x, z)=0, \text { for all } x<y \text { in } P .
$$

If $P$ has a $\hat{0}$, then we write $\mu(x)=\mu(\hat{0}, x)$. Figure 3 shows the intersection poset $L$ of the arrangement $\mathcal{A}$ in $K^{3}$ (for any field $K$ ) defined by $Q_{\mathcal{A}}(x)=x y z(x+y)$, together with the value $\mu(x)$ for all $x \in L$.

A important application of the Möbius function is the Möbius inversion formula. The best way to understand this result (though it does have a simple direct proof) requires the machinery of incidence algebras. Let $\mathcal{J}(P)=\mathcal{J}(P, K)$ denote 
the vector space of all functions $f: \operatorname{Int}(P) \rightarrow K$. Write $f(x, y)$ for $f([x, y])$. For $f, g \in \mathcal{J}(P)$, define the product $f g \in \mathcal{J}(P)$ by

$$
f g(x, y)=\sum_{x \leq z \leq y} f(x, z) g(z, y) .
$$

It is easy to see that this product makes $\mathcal{J}(P)$ an associative $\mathbb{Q}$-algebra, with multiplicative identity $\delta$ given by

$$
\delta(x, y)= \begin{cases}1, & x=y \\ 0, & x<y\end{cases}
$$

Define the zeta function $\zeta \in \mathcal{J}(P)$ of $P$ by $\zeta(x, y)=1$ for all $x \leq y$ in $P$. Note that the Möbius function $\mu$ is an element of $\mathcal{J}(P)$. The definition of $\mu$ (Definition 1.2) is equivalent to the relation $\mu \zeta=\delta$ in $\mathcal{J}(P)$. In any finite-dimensional algebra over a field, one-sided inverses are two-sided inverses, so $\mu=\zeta^{-1}$ in $\mathcal{J}(P)$.

Theorem 1.1. Let $P$ be a finite poset with Möbius function $\mu$, and let $f, g: P \rightarrow K$. Then the following two conditions are equivalent:

$$
\begin{aligned}
f(x) & =\sum_{y \geq x} g(y), \text { for all } x \in P \\
g(x) & =\sum_{y \geq x} \mu(x, y) f(y), \text { for all } x \in P .
\end{aligned}
$$

Proof. The set $K^{P}$ of all functions $P \rightarrow K$ forms a vector space on which $\mathcal{J}(P)$ acts (on the left) as an algebra of linear transformations by

$$
(\xi f)(x)=\sum_{y \geq x} \xi(x, y) f(y),
$$

where $f \in K^{P}$ and $\xi \in \mathcal{J}(P)$. The Möbius inversion formula is then nothing but the statement

$$
\zeta f=g \Leftrightarrow f=\mu g .
$$

We now come to the main concept of this section.

Definition 1.3. The characteristic polynomial $\chi_{\mathcal{A}}(t)$ of the arrangement $\mathcal{A}$ is defined by

$$
\chi_{\mathcal{A}}(t)=\sum_{x \in L(\mathcal{A})} \mu(x) t^{\operatorname{dim}(x)}
$$

For instance, if $\mathcal{A}$ is the arrangement of Figure 3, then

$$
\chi_{\mathcal{A}}(t)=t^{3}-4 t^{2}+5 t-2=(t-1)^{2}(t-2) .
$$

Note that we have immediately from the definition of $\chi_{\mathcal{A}}(t)$, where $\mathcal{A}$ is in $K^{n}$, that

$$
\chi_{\mathcal{A}}(t)=t^{n}-(\# \mathcal{A}) t^{n-1}+\cdots .
$$

Example 1.4. Consider the coordinate hyperplane arrangement $\mathcal{A}$ with defining polynomial $Q_{\mathcal{A}}(x)=x_{1} x_{2} \cdots x_{n}$. Every subset of the hyperplanes in $\mathcal{A}$ has a different nonempty intersection, so $L(\mathcal{A})$ is isomorphic to the boolean algebra $B_{n}$ of all subsets of $[n]=\{1,2, \ldots, n\}$, ordered by inclusion.

Proposition 1.2. Let $\mathcal{A}$ be given by the above example. Then $\chi_{\mathcal{A}}(t)=(t-1)^{n}$. 
Proof. The computation of the Möbius function of a boolean algebra is a standard result in enumerative combinatorics with many proofs. We will give here a naive proof from first principles. Let $y \in L(\mathcal{A}), r(y)=k$. We claim that

$$
\mu(y)=(-1)^{k} .
$$

The assertion is clearly true for $\operatorname{rk}(y)=0$, when $y=\hat{0}$. Now let $y>\hat{0}$. We need to show that

$$
\sum_{x \leq y}(-1)^{\mathrm{rk}(x)}=0 .
$$

The number of $x$ such that $x \leq y$ and $\operatorname{rk}(x)=i$ is $\left(\begin{array}{c}k \\ i\end{array}\right)$, so (5) is equivalent to the well-known identity $\sum_{i=0}^{k}(-1)^{i}\left(\begin{array}{l}k \\ i\end{array}\right)=0$ for $k>0$ (easily proved by substituting $q=-1$ in the binomial expansion of $\left.(q+1)^{k}\right)$. 


\section{Exercises}

We will (subjectively) indicate the difficulty level of each problem as follows:

[1] easy: most students should be able to solve it

[2] moderately difficult: many students should be able to solve it

[3] difficult: a few students should be able to solve it

[4] horrendous: no students should be able to solve it (without already knowing how)

[5] unsolved.

Further gradations are indicated by + and - . Thus a $[3-]$ problem is about the most difficult that makes a reasonable homework exercise, and a [5-] problem is an unsolved problem that has received little attention and may not be too difficult.

Note. Unless explicitly stated otherwise, all graphs, posets, lattices, etc., are assumed to be finite.

(1) [2] Show that every region $R$ of an arrangement $\mathcal{A}$ in $\mathbb{R}^{n}$ is an open convex set. Deduce that $R$ is homeomorphic to the interior of an $n$-dimensional ball.

(2) $[1+]$ Let $\mathcal{A}$ be an arrangement and $\operatorname{ess}(\mathcal{A})$ its essentialization. Show that

$$
t^{\operatorname{dim}(\operatorname{ess}(\mathcal{A}))} \chi_{\mathcal{A}}(t)=t^{\operatorname{dim}(\mathcal{A})} \chi_{\operatorname{ess}(\mathcal{A})}(t) .
$$

(3) $[2+]$ Let $\mathcal{A}$ be the arrangement in $\mathbb{R}^{n}$ with equations

$$
x_{1}=x_{2}, x_{2}=x_{3}, \ldots, x_{n-1}=x_{n}, x_{n}=x_{1} .
$$

Compute the characteristic polynomial $\chi_{\mathcal{A}}(t)$, and compute the number $r(\mathcal{A})$ of regions of $\mathcal{A}$.

(4) $[2+]$ Let $\mathcal{A}$ be an arrangement in $\mathbb{R}^{n}$ with $m$ hyperplanes. Find the maximum possible number $f(n, m)$ of regions of $\mathcal{A}$.

(5) [2] Let $\mathcal{A}$ be an arrangement in the $n$-dimensional vector space $V$ whose normals span a subspace $W$, and let $\mathcal{B}$ be another arrangement in $V$ whose normals span a subspace $Y$. Suppose that $W \cap Y=\{0\}$. Show that

$$
\chi_{\mathcal{A} \cup \mathcal{B}}(t)=t^{-n} \chi_{\mathcal{A}}(t) \chi_{\mathcal{B}}(t) .
$$

(6) [2] Let $\mathcal{A}$ be an arrangment in a vector space $V$. Suppose that $\chi_{\mathcal{A}}(t)$ is divisible by $t^{k}$ but not $t^{k+1}$. Show that $\operatorname{rank}(\mathcal{A})=n-k$.

(7) Let $\mathcal{A}$ be an essential arrangement in $\mathbb{R}^{n}$. Let $\Gamma$ be the union of the bounded faces of $\mathcal{A}$.

(a) [3] Show that $\Gamma$ is contractible.

(b) [2] Show that $\Gamma$ need not be homeomorphic to a closed ball.

(c) $[2+]$ Show that $\Gamma$ need not be starshaped. (A subset $S$ of $\mathbb{R}^{n}$ is starshaped if there is a point $x \in S$ such that for all $y \in S$, the line segment from $x$ to $y$ lies in $S$.)

(d) [3] Show that $\Gamma$ is pure, i.e., all maximal faces of $\Gamma$ have the same dimension. (This was an open problem solved by Xun Dong at the PCMI Summer Session in Geometric Combinatorics, July 11-31, 2004.)

(e) [5] Suppose that $\mathcal{A}$ is in general position. Is $\Gamma$ homeomorphic to an $n$ dimensional closed ball? 
LECTURE 2

Properties of the intersection poset and graphical arrangements

\subsection{Properties of the intersection poset}

Let $\mathcal{A}$ be an arrangement in the vector space $V$. A subarrangement of $\mathcal{A}$ is a subset $\mathcal{B} \subseteq \mathcal{A}$. Thus $\mathcal{B}$ is also an arrangement in $V$. If $x \in L(\mathcal{A})$, define the subarrangement $\mathcal{A}_{x} \subseteq \mathcal{A}$ by

$$
\mathcal{A}_{x}=\{H \in \mathcal{A}: x \subseteq H\} .
$$

Also define an arrangement $\mathcal{A}^{x}$ in the affine subspace $x \in L(\mathcal{A})$ by

$$
\mathcal{A}^{x}=\left\{x \cap H \neq \emptyset: H \in \mathcal{A}-\mathcal{A}_{x}\right\} .
$$

Note that if $x \in L(\mathcal{A})$, then

$$
\begin{aligned}
& L\left(\mathcal{A}_{x}\right) \cong \Lambda_{x}:=\{y \in L(\mathcal{A}): y \leq x\} \\
& L\left(\mathcal{A}^{x}\right) \cong V_{x}:=\{y \in L(\mathcal{A}): y \geq x\}
\end{aligned}
$$

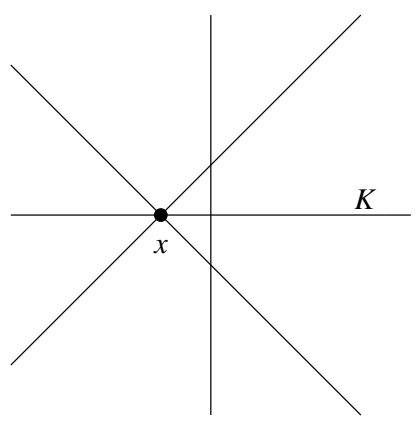

A

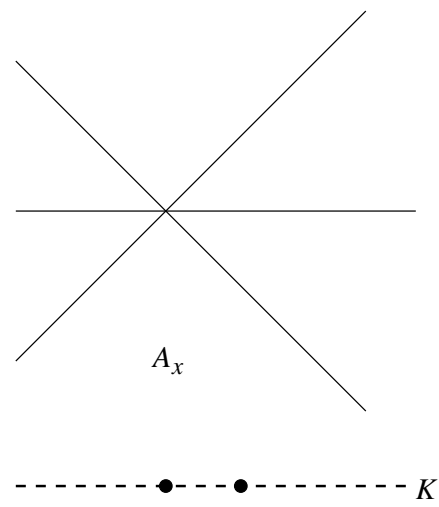

$A^{K}$

Choose $H_{0} \in \mathcal{A}$. Let $\mathcal{A}^{\prime}=\mathcal{A}-\left\{H_{0}\right\}$ and $\mathcal{A}^{\prime \prime}=\mathcal{A}^{H_{0}}$. We call $\left(\mathcal{A}, \mathcal{A}^{\prime}, \mathcal{A}^{\prime \prime}\right)$ a triple of arrangements with distinguished hyperplane $H_{0}$. 

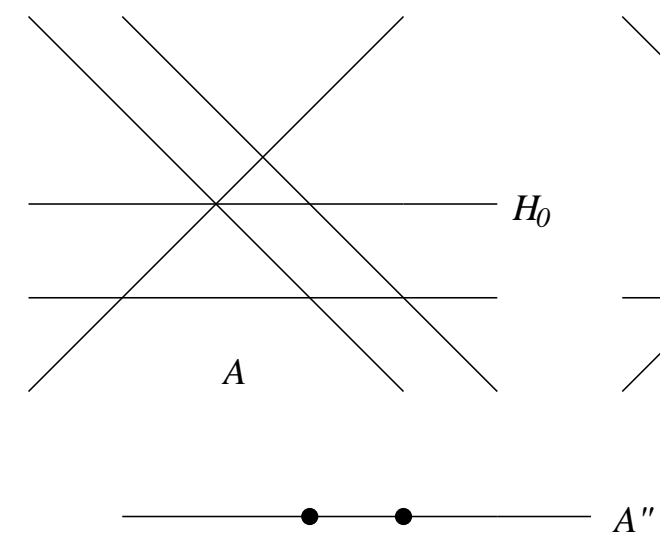

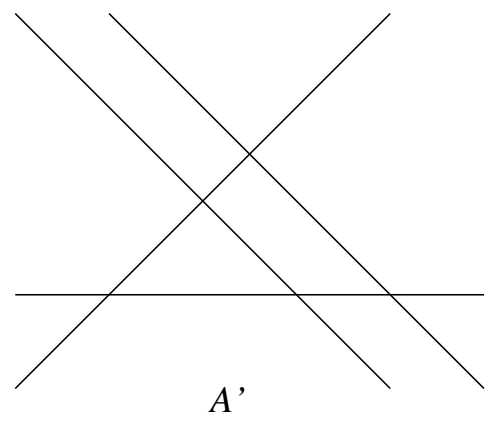

$A^{\prime \prime}$

The main goal of this section is to give a formula in terms of $\chi_{\mathcal{A}}(t)$ for $r(\mathcal{A})$ and $b(\mathcal{A})$ when $K=\mathbb{R}$ (Theorem 2.5). We first establish recurrences for these two quantities.

Lemma 2.1. Let $\left(\mathcal{A}, \mathcal{A}^{\prime}, \mathcal{A}^{\prime \prime}\right)$ be a triple of real arrangements with distinguished hyperplane $H_{0}$. Then

$$
\begin{aligned}
r(\mathcal{A}) & =r\left(\mathcal{A}^{\prime}\right)+r\left(\mathcal{A}^{\prime \prime}\right) \\
b(\mathcal{A}) & =\left\{\begin{aligned}
b\left(\mathcal{A}^{\prime}\right)+b\left(\mathcal{A}^{\prime \prime}\right), & \text { if } \operatorname{rank}(\mathcal{A})=\operatorname{rank}\left(\mathcal{A}^{\prime}\right) \\
0, & \text { if } \operatorname{rank}(\mathcal{A})=\operatorname{rank}\left(\mathcal{A}^{\prime}\right)+1 .
\end{aligned}\right.
\end{aligned}
$$

$\operatorname{Note}$. If $\operatorname{rank}(\mathcal{A})=\operatorname{rank}\left(\mathcal{A}^{\prime}\right)$, then $\operatorname{also} \operatorname{rank}(\mathcal{A})=1+\operatorname{rank}\left(\mathcal{A}^{\prime \prime}\right)$. The figure below illustrates the situation when $\operatorname{rank}(\mathcal{A})=\operatorname{rank}\left(\mathcal{A}^{\prime}\right)+1$.

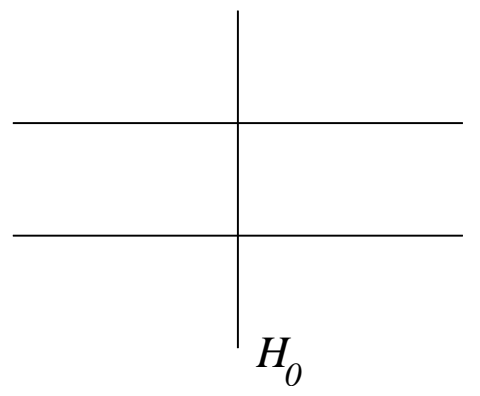

Proof. Note that $r(\mathcal{A})$ equals $r\left(\mathcal{A}^{\prime}\right)$ plus the number of regions of $\mathcal{A}^{\prime}$ cut into two regions by $H_{0}$. Let $R^{\prime}$ be such a region of $\mathcal{A}^{\prime}$. Then $R^{\prime} \cap H_{0} \in \mathcal{R}\left(\mathcal{A}^{\prime \prime}\right)$. Conversely, if $R^{\prime \prime} \in \mathcal{R}\left(\mathcal{A}^{\prime \prime}\right)$ then points near $R^{\prime \prime}$ on either side of $H_{0}$ belong to the same region $R^{\prime} \in \mathcal{R}\left(\mathcal{A}^{\prime}\right)$, since any $H \in \mathcal{R}\left(\mathcal{A}^{\prime}\right)$ separating them would intersect $R^{\prime \prime}$. Thus $R^{\prime}$ is cut in two by $H_{0}$. We have established a bijection between regions of $\mathcal{A}^{\prime}$ cut into two by $H_{0}$ and regions of $\mathcal{A}^{\prime \prime}$, establishing the first recurrence.

The second recurrence is proved analogously; the details are omitted.

We now come to the fundamental recursive property of the characteristic polynomial.

Lemma 2.2. (Deletion-Restriction) Let $\left(\mathcal{A}, \mathcal{A}^{\prime}, \mathcal{A}^{\prime \prime}\right)$ be a triple of real arrangements. Then

$$
\chi_{\mathcal{A}}(t)=\chi_{\mathcal{A}^{\prime}}(t)-\chi_{\mathcal{A}^{\prime \prime}}(t)
$$



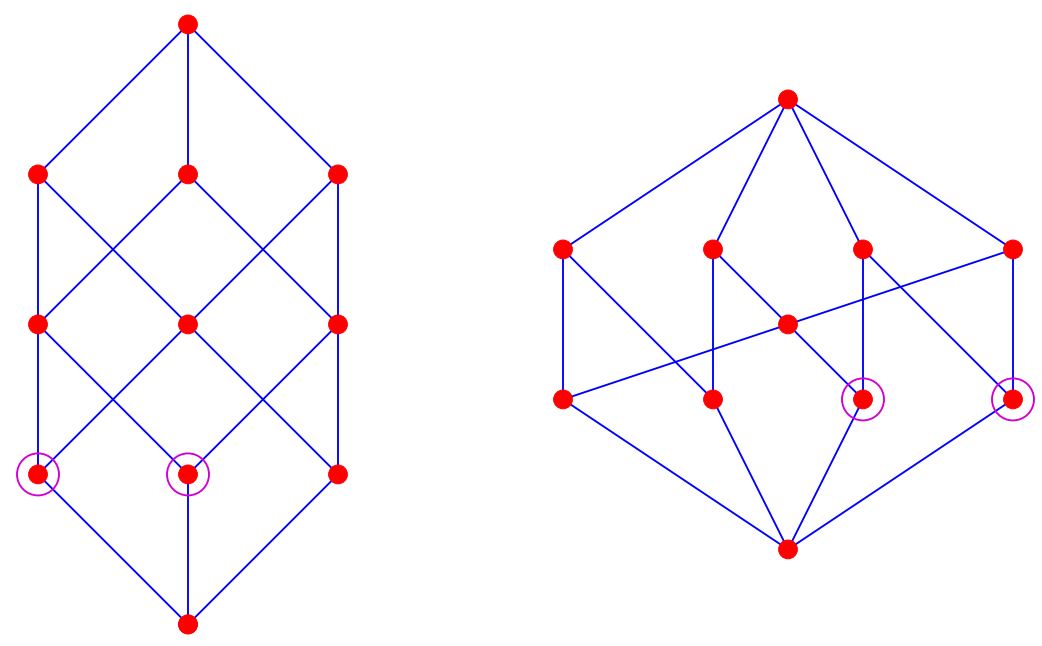

Figure 1. Two non-lattices

For the proof of this lemma, we will need some tools. (A more elementary proof could be given, but the tools will be useful later.)

Let $P$ be a poset. An upper bound of $x, y \in P$ is an element $z \in P$ satisfying $z \geq x$ and $z \geq y$. A least upper bound or join of $x$ and $y$, denoted $x \vee y$, is an upper bound $z$ such that $z \leq z^{\prime}$ for all upper bounds $z^{\prime}$. Clearly if $x \vee y$ exists, then it is unique. Similarly define a lower bound of $x$ and $y$, and a greatest lower bound or meet, denoted $x \wedge y$. A lattice is a poset $L$ for which any two elements have a meet and join. A meet-semilattice is a poset $P$ for which any two elements have a meet. Dually, a join-semilattice is a poset $P$ for which any two elements have a join. Figure 1 shows two non-lattices, with a pair of elements circled which don't have a join.

Lemma 2.3. A finite meet-semilattice $L$ with a unique maximal element $\hat{1}$ is a lattice. Dually, a finite join-semilattice $L$ with a unique minimal element $\hat{0}$ is a lattice.

Proof. Let $L$ be a finite meet-semilattice. If $x, y \in L$ then the set of upper bounds of $x, y$ is nonempty since $\hat{1}$ is an upper bound. Hence

$$
x \vee y=\bigwedge_{\substack{z \geq x \\ z \geq y}} z .
$$

The statement for join-semilattices is by "duality," i.e., interchanging $\leq$ with $\geq$, and $\wedge$ with $\vee$.

The reader should check that Lemma 2.3 need not hold for infinite semilattices.

Proposition 2.3. Let $\mathcal{A}$ be an arrangement. Then $L(\mathcal{A})$ is a meet-semilattice. In particular, every interval $[x, y]$ of $L(\mathcal{A})$ is a lattice. Moreover, $L(\mathcal{A})$ is a lattice if and only if $\mathcal{A}$ is central.

Proof. If $\bigcap_{H \in \mathcal{A}} H=\emptyset$, then adjoin $\emptyset$ to $L(\mathcal{A})$ as the unique maximal element, obtaining the augmented intersection poset $L^{\prime}(\mathcal{A})$. In $L^{\prime}(\mathcal{A})$ it is clear that $x \vee y=$ $x \cap y$. Hence $L^{\prime}(\mathcal{A})$ is a join-semilattice. Since it has a $\hat{0}$, it is a lattice by Lemma 2.3 . 
Since $L(\mathcal{A})=L^{\prime}(\mathcal{A})$ or $L(\mathcal{A})=L^{\prime}(\mathcal{A})-\{\hat{1}\}$, it follows that $L(\mathcal{A})$ is always a meetsemilattice, and is a lattice if $\mathcal{A}$ is central. If $\mathcal{A}$ isn't central, then $\bigvee_{x \in L(\mathcal{A})} x$ does not exist, so $L(\mathcal{A})$ is not a lattice.

We now come to a basic formula for the Möbius function of a lattice.

Theorem 2.2. (the Cross-Cut Theorem) Let $L$ be a finite lattice. Let $X$ be a subset of $L$ such that $\hat{0} \notin X$, and such that if $y \in L, y \neq \hat{0}$, then some $x \in X$ satisfies $x \leq y$. Let $N_{k}$ be the number of k-element subsets of $X$ with join $\hat{1}$. Then

$$
\mu_{L}(\hat{0}, \hat{1})=N_{0}-N_{1}+N_{2}-\cdots .
$$

We will prove Theorem 2.2 by an algebraic method. Such a sophisticated proof is unnecessary, but the machinery we develop will be used later (Theorem 4.13). Let $L$ be a finite lattice and $K$ a field. The Möbius algebra of $L$, denoted $A(L)$, is the semigroup algebra of $L$ over $K$ with respect to the operation $\vee$. (Sometimes the operation is taken to be $\wedge$ instead of $\vee$, but for our purposes, $\vee$ is more convenient.) In other words, $A(L)=K L$ (the vector space with basis $L$ ) as a vector space. If $x, y \in L$ then we define $x y=x \vee y$. Multiplication is extended to all of $A(L)$ by bilinearity (or distributivity). Algebraists will recognize that $A(L)$ is a finite-dimensional commutative algebra with a basis of idempotents, and hence is isomorphic to $K^{\# L}$ (as an algebra). We will show this by exhibiting an explicit isomorphism $A(L) \stackrel{\cong}{\rightrightarrows} K^{\# L}$. For $x \in L$, define

$$
\sigma_{x}=\sum_{y \geq x} \mu(x, y) y \in A(L),
$$

where $\mu$ denotes the Möbius function of $L$. Thus by the Möbius inversion formula,

$$
x=\sum_{y \geq x} \sigma_{y}, \text { for all } x \in L .
$$

Equation (9) shows that the $\sigma_{x}$ 's $\operatorname{span} A(L)$. Since $\#\left\{\sigma_{x}: x \in L\right\}=\# L=$ $\operatorname{dim} A(L)$, it follows that the $\sigma_{x}$ 's form a basis for $A(L)$.

Theorem 2.3. Let $x, y \in L$. Then $\sigma_{x} \sigma_{y}=\delta_{x y} \sigma_{x}$, where $\delta_{x y}$ is the Kronecker delta. In other words, the $\sigma_{x}$ 's are orthogonal idempotents. Hence

$$
A(L)=\bigoplus_{x \in L} K \cdot \sigma_{x} \quad \text { (algebra direct sum). }
$$

Proof. Define a $K$-algebra $A^{\prime}(L)$ with basis $\left\{\sigma_{x}^{\prime}: x \in L\right\}$ and multiplication $\sigma_{x}^{\prime} \sigma_{y}^{\prime}=\delta_{x y} \sigma_{x}^{\prime}$. For $x \in L$ set $x^{\prime}=\sum_{s \geq x} \sigma_{s}^{\prime}$. Then

$$
\begin{aligned}
x^{\prime} y^{\prime} & =\left(\sum_{s \geq x} \sigma_{s}^{\prime}\right)\left(\sum_{t \geq y} \sigma_{t}^{\prime}\right) \\
& =\sum_{\substack{s \geq x \\
s \geq y}} \sigma_{s}^{\prime} \\
& =\sum_{s \geq x \vee y} \sigma_{s}^{\prime} \\
& =(x \vee y)^{\prime} .
\end{aligned}
$$

Hence the linear transformation $\varphi: A(L) \rightarrow A^{\prime}(L)$ defined by $\varphi(x)=x^{\prime}$ is an algebra isomorphism. Since $\varphi\left(\sigma_{x}\right)=\sigma_{x}^{\prime}$, it follows that $\sigma_{x} \sigma_{y}=\delta_{x y} \sigma_{x}$. 
Note. The algebra $A(L)$ has a multiplicative identity, viz., $1=\hat{0}=\sum_{x \in L} \sigma_{x}$.

Proof of Theorem 2.2. Let $\operatorname{char}(K)=0$, e.g., $K=\mathbb{Q}$. For any $x \in L$, we have in $A(L)$ that

$$
\hat{0}-x=\sum_{y \geq \hat{0}} \sigma_{y}-\sum_{y \geq x} \sigma_{y}=\sum_{y \geq x} \sigma_{y} .
$$

Hence by the orthogonality of the $\sigma_{y}$ 's we have

$$
\prod_{x \in X}(\hat{0}-x)=\sum_{y} \sigma_{y}
$$

where $y$ ranges over all elements of $L$ satisfying $y \geq x$ for all $x \in X$. By hypothesis, the only such element is $\hat{0}$. Hence

$$
\prod_{x \in X}(\hat{0}-x)=\sigma_{\hat{0}} .
$$

If we now expand both sides as linear combinations of elements of $L$ and equate coefficients of $\hat{1}$, the result follows.

Note. In a finite lattice $L$, an atom is an element covering $\hat{0}$. Let $T$ be the set of atoms of $L$. Then a set $X \subseteq L-\{\hat{0}\}$ satisfies the hypotheses of Theorem 2.2 if and only if $T \subseteq X$. Thus the simplest choice of $X$ is just $X=T$.

Example 2.5. Let $L=B_{n}$, the boolean algebra of all subsets of $[n]$. Let $X=T=$ $\{\{i\}: i \in[n]\}$. Then $N_{0}=N_{1}=\cdots=N_{n-1}=0, N_{n}=1$. Hence $\mu(\hat{0}, \hat{1})=(-1)^{n}$, agreeing with Proposition 1.2.

We will use the Crosscut Theorem to obtain a formula for the characteristic polynomial of an arrangement $\mathcal{A}$. Extending slightly the definition of a central arrangement, call any subset $\mathcal{B}$ of $\mathcal{A}$ central if $\bigcap_{H \in \mathcal{B}} H \neq \emptyset$. The following result is due to Hassler Whitney for linear arrangements. Its easy extension to arbitrary arrangements appears in [24, Lemma 2.3.8].

Theorem 2.4. (Whitney's theorem) Let $\mathcal{A}$ be an arrangement in an $n$-dimensional vector space. Then

$$
\chi_{\mathcal{A}}(t)=\sum_{\substack{\mathcal{B} \subseteq \mathcal{A} \\ \mathcal{B} \text { central }}}(-1)^{\# \mathcal{B}} t^{n-\operatorname{rank}(\mathcal{B})} .
$$

Example 2.6. Let $\mathcal{A}$ be the arrangement in $\mathbb{R}^{2}$ shown below.

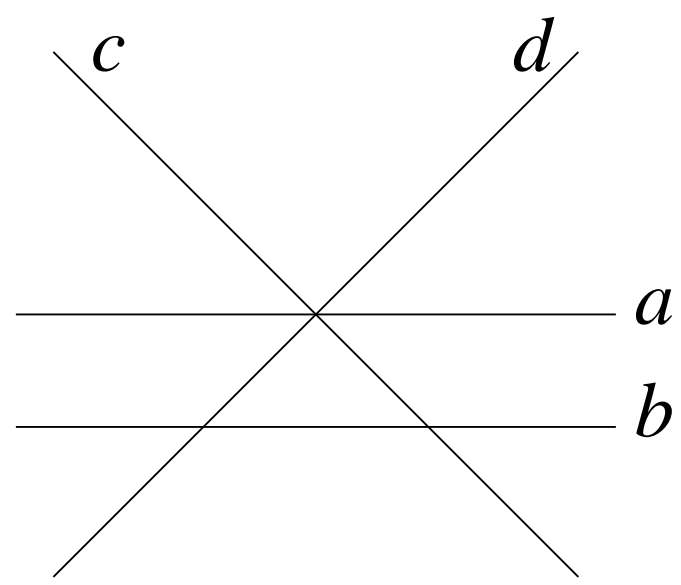


The following table shows all central subsets $\mathcal{B}$ of $\mathcal{A}$ and the values of $\# \mathcal{B}$ and $\operatorname{rank}(\mathcal{B})$

\begin{tabular}{r|cc}
$\mathcal{B}$ & $\# \mathcal{B}$ & $\operatorname{rank}(\mathcal{B})$ \\
\hline$\emptyset$ & 0 & 0 \\
$a$ & 1 & 1 \\
$b$ & 1 & 1 \\
$c$ & 1 & 1 \\
$d$ & 1 & 1 \\
$a c$ & 2 & 2 \\
$a d$ & 2 & 2 \\
$b c$ & 2 & 2 \\
$b d$ & 2 & 2 \\
$c d$ & 2 & 2 \\
$a c d$ & 3 & 2
\end{tabular}

It follows that $\chi_{\mathcal{A}}(t)=t^{2}-4 t+(5-1)=t^{2}-4 t+4$.

Proof of Theorem 2.4. Let $z \in L(\mathcal{A})$. Let

$$
\Lambda_{z}=\{x \in L(\mathcal{A}): x \leq z\},
$$

the principal order ideal generated by $z$. Recall the definition

$$
\mathcal{A}_{z}=\{H \in \mathcal{A}: H \leq z(\text { i.e., } z \subseteq H)\} .
$$

By the Crosscut Theorem (Theorem 2.2), we have

$$
\mu(z)=\sum_{k}(-1)^{k} N_{k}(z),
$$

where $N_{k}(z)$ is the number of $k$-subsets of $\mathcal{A}_{z}$ with join $z$. In other words,

$$
\mu(z)=\sum_{\substack{\mathcal{B} \subseteq \mathcal{A}_{z} \\ z=\bigcap_{H \in \mathcal{B}} H}}(-1)^{\# \mathcal{B}} .
$$

Note that $z=\bigcap_{H \in \mathcal{B}} H$ implies that $\operatorname{rank}(\mathcal{B})=n-\operatorname{dim} z$. Now multiply both sides by $t^{\operatorname{dim}(z)}$ and sum over $z$ to obtain equation (10).

We have now assembled all the machinery necessary to prove the DeletionRestriction Lemma (Lemma 2.2) for $\chi_{\mathcal{A}}(t)$.

Proof of Lemma 2.2. Let $H_{0} \in \mathcal{A}$ be the hyperplane defining the triple $\left(\mathcal{A}, \mathcal{A}^{\prime}, \mathcal{A}^{\prime \prime}\right)$. Split the sum on the right-hand side of (10) into two sums, depending on whether $H_{0} \notin \mathcal{B}$ or $H_{0} \in \mathcal{B}$. In the former case we get

$$
\sum_{\substack{H_{0} \notin \mathcal{B} \subseteq \mathcal{A} \\ \mathcal{B} \text { central }}}(-1)^{\# \mathcal{B}} t^{n-\operatorname{rank}(\mathcal{B})}=\chi_{\mathcal{A}^{\prime}}(t) .
$$

In the latter case, set $\mathcal{B}_{1}=\left(\mathcal{B}-\left\{H_{0}\right\}\right)^{H_{0}}$, a central arrangement in $H_{0} \cong K^{n-1}$ and a subarrangement of $\mathcal{A}^{H_{0}}=\mathcal{A}^{\prime \prime}$. Since $\# \mathcal{B}_{1}=\# \mathcal{B}-1$ and $\operatorname{rank}\left(\mathcal{B}_{1}\right)=\operatorname{rank}(\mathcal{B})-1$, we get

$$
\begin{aligned}
\sum_{\substack{H_{0} \in \mathcal{B} \subseteq \mathcal{A} \\
\text { central }}}(-1)^{\# \mathcal{B}} t^{n-\operatorname{rank}(\mathcal{B})} & =\sum_{\mathcal{B}_{1} \in \mathcal{A}^{\prime \prime}}(-1)^{\# \mathcal{B}_{1}+1} t^{(n-1)-\operatorname{rank}\left(\mathcal{B}_{1}\right)} \\
& =-\chi_{\mathcal{A}^{\prime \prime}}(t),
\end{aligned}
$$

and the proof follows. 


\subsection{The number of regions}

The next result is perhaps the first major theorem in the subject of hyperplane arrangements, due to Thomas Zaslavsky in 1975.

Theorem 2.5. Let $\mathcal{A}$ be an arrangement in an $n$-dimensional real vector space. Then

$$
\begin{aligned}
r(\mathcal{A}) & =(-1)^{n} \chi_{\mathcal{A}}(-1) \\
b(\mathcal{A}) & =(-1)^{\operatorname{rank}(\mathcal{A})} \chi_{\mathcal{A}}(1) .
\end{aligned}
$$

First proof. Equation (11) holds for $\mathcal{A}=\emptyset$, since $r(\emptyset)=1$ and $\chi_{\emptyset}(t)=t^{n}$. By Lemmas 2.1 and 2.2 , both $r(\mathcal{A})$ and $(-1)^{n} \chi_{\mathcal{A}}(-1)$ satisfy the same recurrence, so the proof follows.

Now consider equation (12). Again it holds for $\mathcal{A}=\emptyset$ since $b(\emptyset)=1$. (Recall that $b(\mathcal{A})$ is the number of relatively bounded regions. When $\mathcal{A}=\emptyset$, the entire ambient space $\mathbb{R}^{n}$ is relatively bounded.) Now

$$
\chi_{\mathcal{A}}(1)=\chi_{\mathcal{A}^{\prime}}(1)-\chi_{\mathcal{A}^{\prime \prime}}(1) .
$$

Let $d(\mathcal{A})=(-1)^{\operatorname{rank}(\mathcal{A})} \chi_{\mathcal{A}}(1)$. If $\operatorname{rank}(\mathcal{A})=\operatorname{rank}\left(\mathcal{A}^{\prime}\right)=\operatorname{rank}\left(\mathcal{A}^{\prime \prime}\right)+1$, then $d(\mathcal{A})=$ $d\left(\mathcal{A}^{\prime}\right)+d\left(\mathcal{A}^{\prime \prime}\right)$. If $\operatorname{rank}(\mathcal{A})=\operatorname{rank}\left(\mathcal{A}^{\prime}\right)+1$ then $b(\mathcal{A})=0$ [why?] and $L\left(\mathcal{A}^{\prime}\right) \cong L\left(\mathcal{A}^{\prime \prime}\right)$ [why?]. Thus from Lemma 2.2 we have $d(\mathcal{A})=0$. Hence in all cases $b(\mathcal{A})$ and $d(\mathcal{A})$ satisfy the same recurrence, so $b(\mathcal{A})=d(\mathcal{A})$.

Second proof. Our second proof of Theorem 2.5 is based on Möbius inversion and some instructive topological considerations. For this proof we assume basic knowledge of the Euler characteristic $\psi(\Delta)$ of a topological space $\Delta$. (Standard notation is $\chi(\Delta)$, but this would cause too much confusion with the characteristic polynomial.) In particular, if $\Delta$ is suitably decomposed into cells with $f_{i}$ $i$-dimensional cells, then

$$
\psi(\Delta)=f_{0}-f_{1}+f_{2}-\cdots .
$$

We take (13) as the definition of $\psi(\Delta)$. For "nice" spaces and decompositions, it is independent of the decomposition. In particular, $\psi\left(\mathbb{R}^{n}\right)=(-1)^{n}$. Write $\bar{R}$ for the closure of a region $R \in \mathcal{R}(\mathcal{A})$.

Definition 2.4. A (closed) face of a real arrangement $\mathcal{A}$ is a set $\emptyset \neq F=\bar{R} \cap x$, where $R \in \mathcal{R}(\mathcal{A})$ and $x \in L(\mathcal{A})$.

If we regard $\bar{R}$ as a convex polyhedron (possibly unbounded), then a face of $\mathcal{A}$ is just a face of some $\bar{R}$ in the usual sense of the face of a polyhedron, i.e., the intersection of $\bar{R}$ with a supporting hyperplane. In particular, each $\bar{R}$ is a face of $\mathcal{A}$. The dimension of a face $F$ is defined by

$$
\operatorname{dim}(F)=\operatorname{dim}(\operatorname{aff}(F)),
$$

where $\operatorname{aff}(F)$ denotes the affine span of $F$. A $k$-face is a $k$-dimensional face of $\mathcal{A}$. For instance, the arrangement below has three 0 -faces (vertices), nine 1 -faces, and seven 2 -faces (equivalently, seven regions). Hence $\psi\left(\mathbb{R}^{2}\right)=3-9+7=1$. 


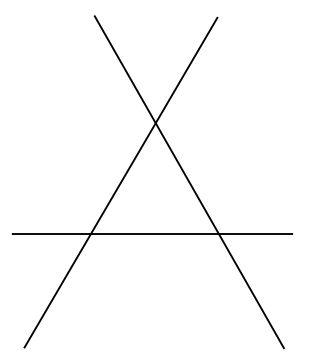

Write $\mathcal{F}(\mathcal{A})$ for the set of faces of $\mathcal{A}$, and let relint denote relative interior. Then

$$
\mathbb{R}^{n}=\bigsqcup_{F \in \mathcal{F}(\mathcal{A})} \operatorname{relint}(F),
$$

where $\bigsqcup$ denotes disjoint union. If $f_{k}(\mathcal{A})$ denotes the number of $k$-faces of $\mathcal{A}$, it follows that

$$
(-1)^{n}=\psi\left(\mathbb{R}^{n}\right)=f_{0}(\mathcal{A})-f_{1}(\mathcal{A})+f_{2}(\mathcal{A})-\cdots .
$$

Every $k$-face is a region of exactly one $\mathcal{A}^{y}$ for $y \in L(\mathcal{A})$. Hence

$$
f_{k}(\mathcal{A})=\sum_{\substack{y \in L(\mathcal{A}) \\ \operatorname{dim}(y)=k}} r\left(\mathcal{A}^{y}\right) .
$$

Multiply by $(-1)^{k}$ and sum over $k$ to get

$$
(-1)^{n}=\psi\left(\mathbb{R}^{n}\right)=\sum_{y \in L(\mathcal{A})}(-1)^{\operatorname{dim}(y)} r\left(\mathcal{A}^{y}\right) .
$$

Replacing $\mathbb{R}^{n}$ by $x \in L(\mathcal{A})$ gives

$$
(-1)^{\operatorname{dim}(x)}=\psi(x)=\sum_{\substack{y \in L(\mathcal{A}) \\ y \geq x}}(-1)^{\operatorname{dim}(y)} r\left(\mathcal{A}^{y}\right) .
$$

Möbius inversion yields

$$
(-1)^{\operatorname{dim}(x)} r\left(\mathcal{A}^{x}\right)=\sum_{\substack{y \in L(\mathcal{A}) \\ y \geq x}}(-1)^{\operatorname{dim}(y)} \mu(x, y) .
$$

Putting $x=\mathbb{R}^{n}$ gives

$$
(-1)^{n} r(\mathcal{A})=\sum_{y \in L(\mathcal{A})}(-1)^{\operatorname{dim}(y)} \mu(y)=\chi_{\mathcal{A}}(-1),
$$

thereby proving (11).

The relatively bounded case (equation (12)) is similar, but with one technical complication. We may assume that $\mathcal{A}$ is essential, since $b(\mathcal{A})=b(\operatorname{ess}(\mathcal{A}))$ and

$$
\chi_{\mathcal{A}}(t)=t^{\operatorname{dim}(\mathcal{A})-\operatorname{dim}(\operatorname{ess}(\mathcal{A}))} \chi_{\operatorname{ess}(\mathcal{A})}(t) .
$$

In this case, the relatively bounded regions are actually bounded. Let

$$
\begin{aligned}
\mathcal{F}_{b}(\mathcal{A}) & =\{F \in \mathcal{F}(\mathcal{A}): F \text { is relatively bounded }\} \\
\Gamma & =\bigcup_{F \in \mathcal{F}_{b}(\mathcal{A})} F .
\end{aligned}
$$

The difficulty lies in computing $\psi(\Gamma)$. Zaslavsky conjectured in 1975 that $\Gamma$ is star-shaped, i.e., there exists $x \in \Gamma$ such that for every $y \in \Gamma$, the line segment 

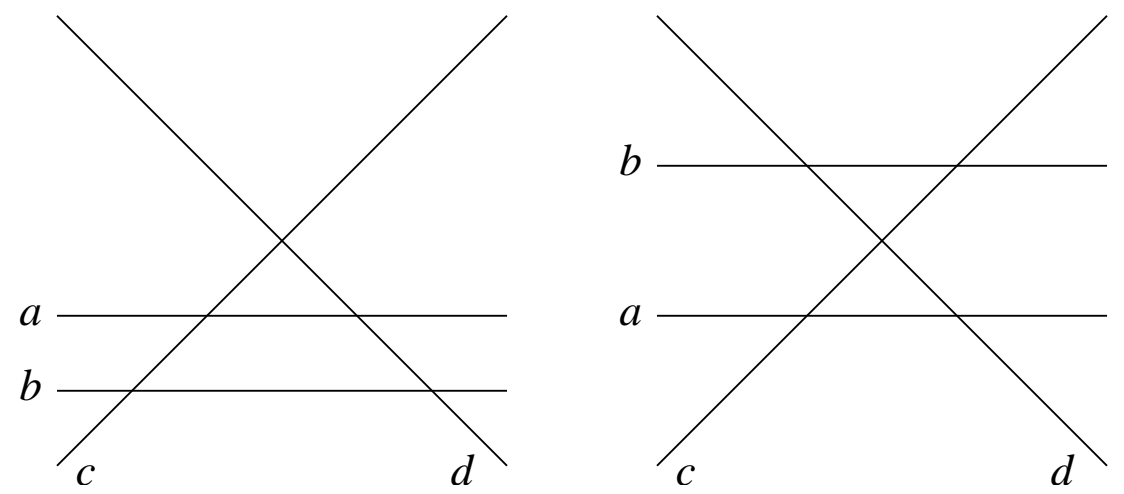

Figure 2. Two arrangements with the same intersection poset

joining $x$ and $y$ lies in $\Gamma$. This would imply that $\Gamma$ is contractible, and hence (since $\Gamma$ is compact when $\mathcal{A}$ is essential) $\psi(\Gamma)=1$. A counterexample to Zaslavsky's conjecture appears as an exercise in [7, Exer. 4.29], but nevertheless Björner and Ziegler showed that $\Gamma$ is indeed contractible. (See [7, Thm. 4.5.7(b)] and Lecture 1, Exercise 7.) The argument just given for $r(\mathcal{A})$ now carries over mutatis mutandis to $b(\mathcal{A})$. There is also a direct argument that $\psi(\Gamma)=1$, circumventing the need to show that $\Gamma$ is contractible. We will omit proving here that $\psi(\Gamma)=1$.

Corollary 2.1. Let $\mathcal{A}$ be a real arrangement. Then $r(\mathcal{A})$ and $b(\mathcal{A})$ depend only on $L(\mathcal{A})$.

Figure 2 shows two arrangements in $\mathbb{R}^{2}$ with different "face structure" but the same $L(\mathcal{A})$. The first arrangement has for instance one triangular and one quadrilateral face, while the second has two triangular faces. Both arrangements, however, have ten regions and two bounded regions.

We now give two basic examples of arrangements and the computation of their characteristic polynomials.

Proposition 2.4. (general position) Let $\mathcal{A}$ be an n-dimensional arrangement of $m$ hyperplanes in general position. Then

$$
\chi_{\mathcal{A}}(t)=t^{n}-m t^{n-1}+\left(\begin{array}{c}
m \\
2
\end{array}\right) t^{n-2}-\cdots+(-1)^{n}\left(\begin{array}{c}
m \\
n
\end{array}\right) .
$$

In particular, if $\mathcal{A}$ is a real arrangement, then

$$
\begin{aligned}
r(\mathcal{A}) & =1+m+\left(\begin{array}{c}
m \\
2
\end{array}\right)+\cdots+\left(\begin{array}{c}
m \\
n
\end{array}\right) \\
b(\mathcal{A}) & =(-1)^{n}\left(1-m+\left(\begin{array}{c}
m \\
2
\end{array}\right)-\cdots+(-1)^{n}\left(\begin{array}{c}
m \\
n
\end{array}\right)\right) \\
& =\left(\begin{array}{c}
m-1 \\
n
\end{array}\right) .
\end{aligned}
$$

Proof. Every $\mathcal{B} \subseteq \mathcal{A}$ with $\# \mathcal{B} \leq n$ defines an element $x_{\mathcal{B}}=\bigcap_{H \in \mathcal{B}} H$ of $L(\mathcal{A})$. Hence $L(\mathcal{A})$ is a truncated boolean algebra:

$$
L(\mathcal{A}) \cong\{S \subseteq[m]: \# S \leq n\},
$$




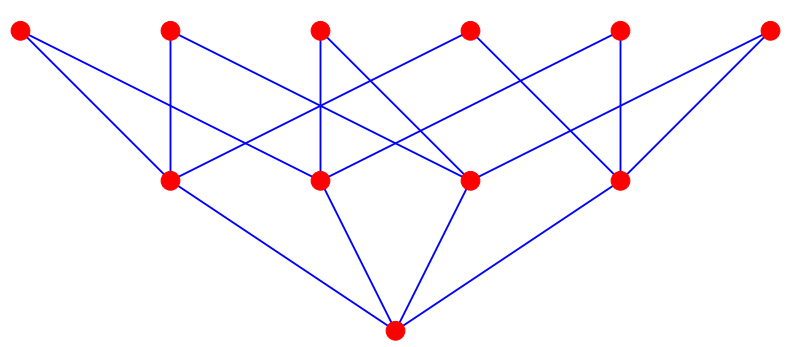

Figure 3. The truncated boolean algebra of rank 2 with four atoms

ordered by inclusion. Figure 3 shows the case $n=2$ and $m=4$, i.e., four lines in general position in $\mathbb{R}^{2}$. If $x \in L(\mathcal{A})$ and $\operatorname{rk}(x)=k$, then $[\hat{0}, x] \cong B_{k}$, a boolean algebra of rank $k$. By equation (4) there follows $\mu(x)=(1)^{k}$. Hence

$$
\begin{aligned}
\chi_{\mathcal{A}}(t) & =\sum_{\substack{S \subseteq[m] \\
\# S \leq n}}(-1)^{\# S} t^{n-\# S} \\
& =t^{n}-m t^{n-1}+\cdots+(-1)^{n}\left(\begin{array}{c}
m \\
n
\end{array}\right) .
\end{aligned}
$$

Note. Arrangements whose hyperplanes are in general position were formerly called free arrangements. Now, however, free arrangements have another meaning discussed in the note following Example 4.11.

Our second example concerns generic translations of the hyperplanes of a linear arrangement. Let $L_{1}, \ldots, L_{m}$ be linear forms, not necessarily distinct, in the variables $v=\left(v_{1}, \ldots, v_{n}\right)$ over the field $K$. Let $\mathcal{A}$ be defined by

$$
L_{1}(v)=a_{1}, \ldots, L_{m}(v)=a_{m},
$$

where $a_{1}, \ldots, a_{m}$ are generic elements of $K$. This means if $H_{i}=\operatorname{ker}\left(L_{i}(v)-a_{i}\right)$, then

$$
H_{i_{1}} \cap \cdots \cap H_{i_{k}} \neq \emptyset \Leftrightarrow L_{i_{1}}, \ldots, L_{i_{k}} \text { are linearly independent. }
$$

For instance, if $K=\mathbb{R}$ and $L_{1}, \ldots, L_{m}$ are defined over $\mathbb{Q}$, then $a_{1}, \ldots, a_{m}$ are generic whenever they are linearly independent over $\mathbb{Q}$.

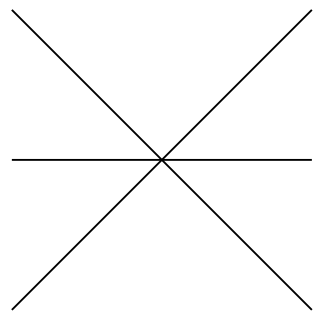

nongeneric

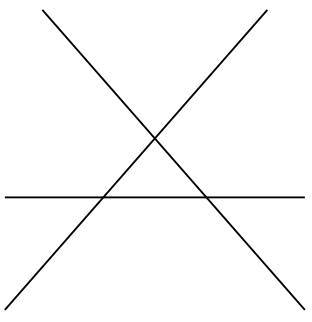

generic

It follows that if $x=H_{i_{1}} \cap \cdots \cap H_{i_{k}} \in L(\mathcal{A})$, then $[\hat{0}, x] \cong B_{k}$. Hence

$$
\chi_{\mathcal{A}}(t)=\sum_{\mathcal{B}}(-1)^{\# \mathcal{B}} t^{n-\# \mathcal{B}}
$$



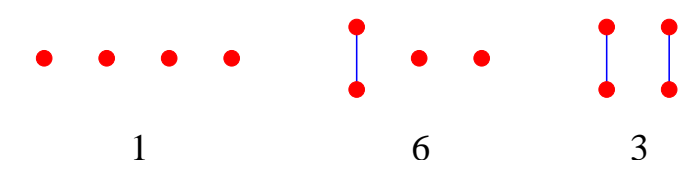

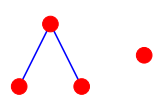

12

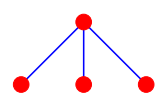

4

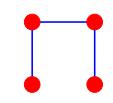

12

Figure 4. The forests on four vertices

where $\mathcal{B}$ ranges over all linearly independent subsets of $\mathcal{A}$. (We say that a set of hyperplanes are linearly independent if their normals are linearly independent.) Thus $\chi_{\mathcal{A}}(t)$, or more precisely $(-t)^{n} \chi_{\mathcal{A}}(-1 / t)$, is the generating function for linearly independent subsets of $L_{1}, \ldots, L_{m}$ according to their number of elements. For instance, if $\mathcal{A}$ is given by Figure 2 (either arrangement) then the linearly independent subsets of hyperplanes are $\emptyset, a, b, c, d, a c, a d, b c, b d, c d$, so $\chi_{\mathcal{A}}(t)=t^{2}-4 t+5$.

Consider the more interesting example $x_{i}-x_{j}=a_{i j}, 1 \leq i<j \leq n$, where the $a_{i j}$ are generic. We could call this arrangement the generic braid arrangement $\mathcal{G}_{n}$. Identify the hyperplane $x_{i}-x_{j}=a_{i j}$ with the edge $i j$ on the vertex set $[n]$. Thus a subset $\mathcal{B} \subseteq \mathcal{G}_{n}$ corresponds to a simple graph $G_{\mathcal{B}}$ on $[n]$. ("Simple" means that there is at most one edge between any two vertices, and no edge from a vertex to itself.) It is easy to see that $\mathcal{B}$ is linearly independent if and only if the graph $G_{\mathcal{B}}$ has no cycles, i.e., is a forest. Hence we obtain the interesting formula

$$
\chi_{\mathcal{S}_{n}}(t)=\sum_{F}(-1)^{e(F)} t^{n-e(F)},
$$

where $F$ ranges over all forests on $[n]$ and $e(F)$ denotes the number of edges of $F$. For instance, the isomorphism types of forests (with the number of distinct labelings written below the forest) on four vertices are given by Figure 4 . Hence

$$
\chi_{\mathcal{S}_{4}}(t)=t^{4}-6 t^{3}+15 t^{2}-16 t .
$$

Equation (11) can be rewritten as

$$
r(\mathcal{A})=\sum_{x \in L(\mathcal{A})}(-1)^{\mathrm{rk}(x)} \mu(x) .
$$

(Theorem 3.10 will show that $(-1)^{\mathrm{rk}(x)} \mu(x)>0$, so we could also write $|\mu(x)|$ for this quantity.) It is easy to extend this result to count faces of $\mathcal{A}$ of all dimensions, not just the top dimension $n$. Let $f_{k}(\mathcal{A})$ denote the number of $k$-faces of the real arrangement $\mathcal{A}$.

Theorem 2.6. We have

$$
\begin{aligned}
f_{k}(\mathcal{A}) & =\sum_{\substack{x \leq y \text { in } L(\mathcal{A}) \\
\operatorname{dim}(x)=k}}(-1)^{\operatorname{dim}(x)-\operatorname{dim}(y)} \mu(x, y) \\
& =\sum_{\substack{x \leq y \text { in } L(\mathcal{A}) \\
\operatorname{dim}(x)=k}}|\mu(x, y)| .
\end{aligned}
$$

Proof. As mentioned above, every face $F$ is a region of a unique $\mathcal{A}^{x}$ for $x \in L(\mathcal{A})$, viz., $x=\operatorname{aff}(F)$. In particular, $\operatorname{dim}(F)=\operatorname{dim}(x)$. Hence if $\operatorname{dim}(F)=k$, then $r\left(\mathcal{A}^{x}\right)$ is the number of $k$-faces of $\mathcal{A}$ contained in $x$. By Theorem 2.5 and equation (7) we get

$$
r\left(\mathcal{A}^{x}\right)=\sum_{y \geq x}(-1)^{\operatorname{dim}(y)-\operatorname{dim}(x)} \mu(x, y),
$$


where we are dealing with the poset $L(\mathcal{A})$. Summing over all $x \in L(\mathcal{A})$ of dimension $k$ yields (15), and (16) then follows from Theorem (3.10) below.

\subsection{Graphical arrangements}

There are close connections between certain invariants of a graph $G$ and an associated arrangement $\mathcal{A}_{G}$. Let $G$ be a simple graph on the vertex set $[n]$. Let $E(G)$ denote the set of edges of $G$, regarded as two-element subsets of $[n]$. Write $i j$ for the edge $\{i, j\}$.

Definition 2.5. The graphical arrangement $\mathcal{A}_{G}$ in $K^{n}$ is the arrangement

$$
x_{i}-x_{j}=0, i j \in E(G) .
$$

Thus a graphical arrangement is simply a subarrangement of the braid arrangement $\mathcal{B}_{n}$. If $G=K_{n}$, the complete graph on [n] (with all possible edges $i j$ ), then $\mathcal{A}_{K_{n}}=\mathcal{B}_{n}$.

Definition 2.6. A coloring of a graph $G$ on $[n]$ is a map $\kappa:[n] \rightarrow \mathbb{P}$. The coloring $\kappa$ is proper if $\kappa(i) \neq \kappa(j)$ whenever $i j \in E(G)$. If $q \in \mathbb{P}$ then let $\chi_{G}(q)$ denote the number of proper colorings $\kappa:[n] \rightarrow[q]$ of $G$, i.e., the number of proper colorings of $G$ whose colors come from $1,2, \ldots, q$. The function $\chi_{G}$ is called the chromatic polynomial of $G$.

For instance, suppose that $G$ is the complete graph $K_{n}$. A proper coloring $\kappa:[n] \rightarrow[q]$ is obtained by choosing a vertex, say 1 , and coloring it in $q$ ways. Then choose another vertex, say 2 , and color it in $q-1$ ways, etc., obtaining

$$
\chi_{K_{n}}(q)=q(q-1) \cdots(q-n+1) .
$$

A similar argument applies to the graph $G$ of Figure 5 . There are $q$ ways to color vertex 1 , then $q-1$ to color vertex 2 , then $q-1$ to color vertex 3, etc., obtaining

$$
\begin{aligned}
\chi_{G}(q) & =q(q-1)(q-1)(q-2)(q-1)(q-1)(q-2)(q-2)(q-3) \\
& =q(q-1)^{4}(q-2)^{3}(q-3) .
\end{aligned}
$$

Unlike the case of the complete graph, in order to obtain this nice product formula one factor at a time only certain orderings of the vertices are suitable. It is not always possible to evaluate the chromatic polynomials "one vertex at a time." For instance, let $H$ be the 4-cycle of Figure 5. If a proper coloring $\kappa:[4] \rightarrow[q]$ satisfies $\kappa(1)=\kappa(3)$, then there are $q$ choices for $\kappa(1)$, then $q-1$ choices each for $\kappa(2)$ and $\kappa(4)$. On the other hand, if $\kappa(1) \neq \kappa(3)$, then there are $q$ choices for $\kappa(1)$, then $q-1$ choices for $\kappa(3)$, and then $q-2$ choices each for $\kappa(2)$ and $\kappa(4)$. Hence

$$
\begin{aligned}
\chi_{H}(q) & =q(q-1)^{2}+q(q-1)(q-2)^{2} \\
& =q(q-1)\left(q^{2}-3 q+3\right) .
\end{aligned}
$$

For further information on graphs whose chromatic polynomial can be evaluated one vertex at a time, see Corollary 4.10 and the note following it.

It is easy to see directly that $\chi_{G}(q)$ is a polynomial function of $q$. Let $e_{i}(G)$ denote the number of surjective proper colorings $\kappa:[n] \rightarrow[i]$ of $G$. We can choose an arbitrary proper coloring $\kappa:[n] \rightarrow[q]$ by first choosing the size $i=\# \kappa([n])$ of its image in $\left(\begin{array}{l}q \\ i\end{array}\right)$ ways, and then choose $\kappa$ in $e_{i}$ ways. Hence

$$
\chi_{G}(q)=\sum_{i=0}^{n} e_{i}\left(\begin{array}{l}
q \\
i
\end{array}\right) .
$$



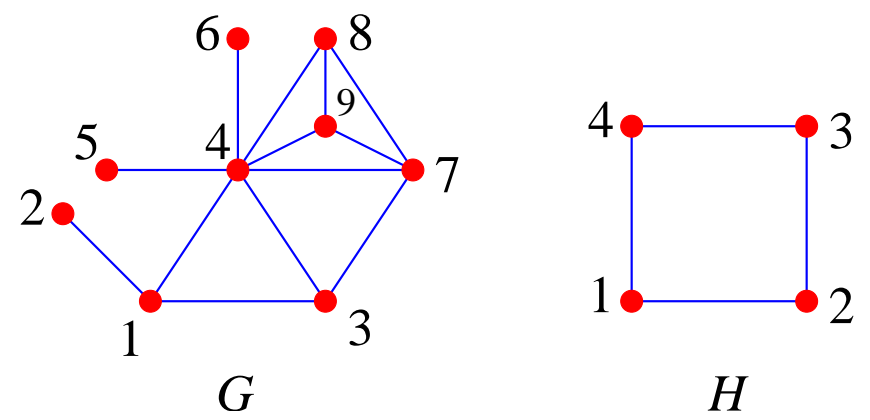

Figure 5. Two graphs

Since $\left(\begin{array}{l}q \\ i\end{array}\right)=q(q-1) \cdots(q-i+1) / i$, a polynomial in $q$ (of degree $i$ ), we see that $\chi_{G}(q)$ is a polynomial. We therefore write $\chi_{G}(t)$, where $t$ is an indeterminate. Moreover, any surjection (= bijection) $\kappa:[n] \rightarrow[n]$ is proper. Hence $e_{n}=n$ !. It follows from equation (18) that $\chi_{G}(t)$ is monic of degree $n$. Using more sophisticated methods we will later derive further properties of the coefficients of $\chi_{G}(t)$.

Theorem 2.7. For any graph $G$, we have $\chi_{\mathcal{A}_{G}}(t)=\chi_{G}(t)$.

First proof. The first proof is based on deletion-restriction (which in the context of graphs is called deletion-contraction). Let $e=i j \in E(G)$. Let $G-e$ (also denoted $G \backslash e$ ) denote the graph $G$ with edge $e$ deleted, and let $G / e$ denote $G$ with the edge $e$ contracted to a point and all multiple edges replaced by a single edge (i.e., whenever there is more than one edge between two vertices, replace these edges by a single edge). (In some contexts we want to keep track of multiple edges, but they are irrelevant in regard to proper colorings.)
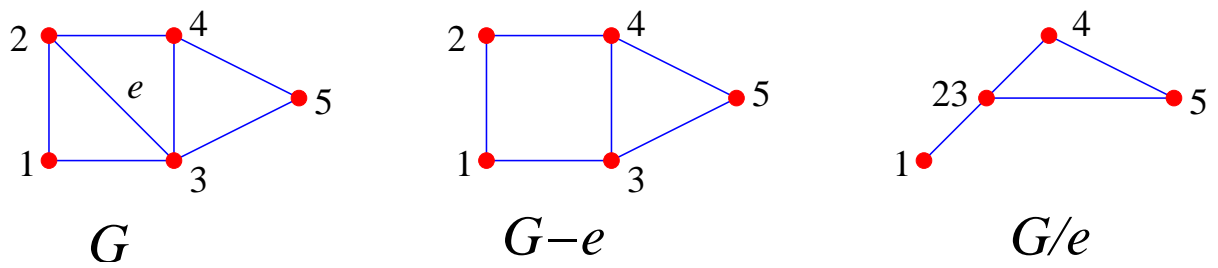

Let $H_{0} \in \mathcal{A}=\mathcal{A}_{G}$ be the hyperplane $x_{i}=x_{j}$. It is clear that $\mathcal{A}-\left\{H_{0}\right\}=\mathcal{A}_{G-e}$. We claim that

$$
\mathcal{A}^{H_{0}}=\mathcal{A}_{G / e},
$$

so by Deletion-Restriction (Lemma 2.2) we have

$$
\chi_{\mathcal{A}_{G}}(t)=\chi_{\mathcal{A}_{G-e}}(t)=\chi_{\mathcal{A}_{G / e}}(t) .
$$

To prove (19), define an affine isomorphism $\varphi: H_{0} \cong \mathbb{R}^{n-1}$ by

$$
\left(x_{1}, x_{2}, \ldots, x_{n}\right) \mapsto\left(x_{1}, \ldots, x_{i}, \ldots, \hat{x_{j}}, \ldots, x_{n}\right),
$$

where $\hat{x_{j}}$ denotes that the $j$ th coordinate is omitted. (Hence the coordinates in $\mathbb{R}^{n-1}$ are $1,2, \ldots, \hat{j}, \ldots, n$.) Write $H_{a b}$ for the hyperplane $x_{a}=x_{b}$ of $\mathcal{A}$. If neither of $a, b$ are equal to $i$ or $j$, then $\varphi\left(H_{a b} \cap H_{0}\right)$ is the hyperplane $x_{a}=x_{b}$ in $\mathbb{R}^{n-1}$. If $a \neq i, j$ then $\varphi\left(H_{i a} \cap H_{0}\right)=\varphi\left(H_{a j} \cap H_{0}\right)$, the hyperplane $x_{a}=x_{i}$ in $\mathbb{R}^{n-1}$. Hence $\varphi$ 

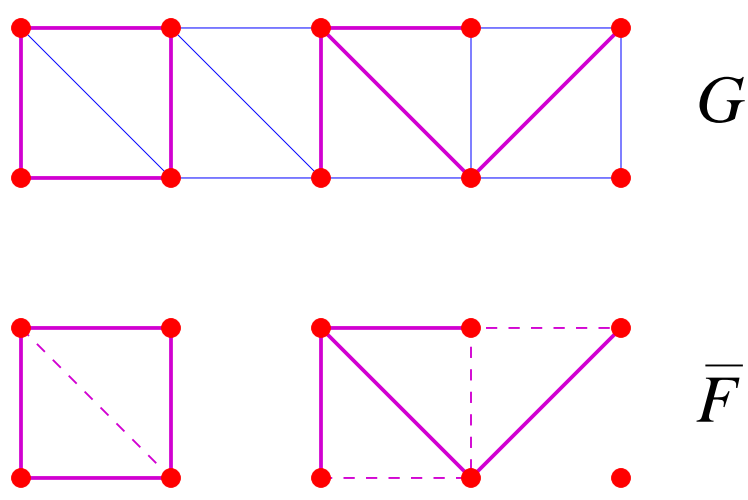

Figure 6. A graph $G$ with edge subset $F$ and closure $\bar{F}$

defines an isomorphism between $\mathcal{A}^{H_{0}}$ and the arrangement $\mathcal{A}_{G / e}$ in $\mathbb{R}^{n-1}$, proving (19).

Let $n \bullet$ denote the graph with $n$ vertices and no edges, and let $\emptyset$ denote the empty arrangement in $\mathbb{R}^{n}$. The theorem will be proved by induction (using Lemma 2.2) if we show:

(a) Initialization: $\chi_{n \bullet}(t)=\chi_{\emptyset}(t)$

(b) Deletion-contraction:

$$
\chi_{G}(t)=\chi_{G-e}(t)-\chi_{G / e}(t)
$$

To prove (a), note that both sides are equal to $t^{n}$. To prove (b), observe that $\chi_{G-e}(q)$ is the number of colorings of $\kappa:[n] \rightarrow[q]$ that are proper except possibly $\kappa(i)=\kappa(j)$, while $\chi_{G / e}(q)$ is the number of colorings $\kappa:[n] \rightarrow[q]$ of $G$ that are proper except that $\kappa(i)=\kappa(j)$.

Our second proof of Theorem 2.7 is based on Möbius inversion. We first obtain a combinatorial description of the intersection lattice $L\left(\mathcal{A}_{G}\right)$. Let $H_{i j}$ denote the hyperplane $x_{i}=x_{j}$ as above, and let $F \subseteq E(G)$. Consider the element $X=$ $\bigcap_{i j \in F} H_{i j}$ of $L\left(\mathcal{A}_{G}\right)$. Thus

$$
\left(x_{1}, \ldots, x_{n}\right) \in X \Leftrightarrow x_{i}=x_{j} \text { whenever } i j \in F .
$$

Let $C_{1}, \ldots, C_{k}$ be the connected components of the spanning subgraph $G_{F}$ of $G$ with edge set $F$. (A subgraph of $G$ is spanning if it contains all the vertices of $G$. Thus if the edges of $F$ do not span all of $G$, we need to include all remaining vertices as isolated vertices of $G_{F}$.) If $i, j$ are vertices of some $C_{m}$, then there is a path from $i$ to $j$ whose edges all belong to $F$. Hence $x_{i}=x_{j}$ for all $\left(x_{1}, \ldots, x_{n}\right) \in X$. On the other hand, if $i$ and $j$ belong to different $C_{m}$ 's, then there is no such path. Let

$$
\bar{F}=\left\{e=i j \in E(G): i, j \in V\left(C_{m}\right) \text { for some } m\right\},
$$

where $V\left(C_{m}\right)$ denotes the vertex set of $C_{m}$. Figure 6 illustrates a graph $G$ with a set $F$ of edges indicated by thickening. The set $\bar{F}$ is shown below $G$, with the additional edges $\bar{F}-F$ not in $F$ drawn as dashed lines.

A partition $\pi$ of a finite set $S$ is a collection $\left\{B_{1}, \ldots, B_{k}\right\}$ of subsets of $S$, called blocks, that are nonempty, pairwise disjoint, and whose union is $S$. The set of all partitions of $S$ is denoted $\Pi_{S}$, and when $S=[n]$ we write simply $\Pi_{n}$ for $\Pi_{[n]}$. It follows from the above discussion that the elements $X_{\pi}$ of $L\left(\mathcal{A}_{G}\right)$ correspond to the 

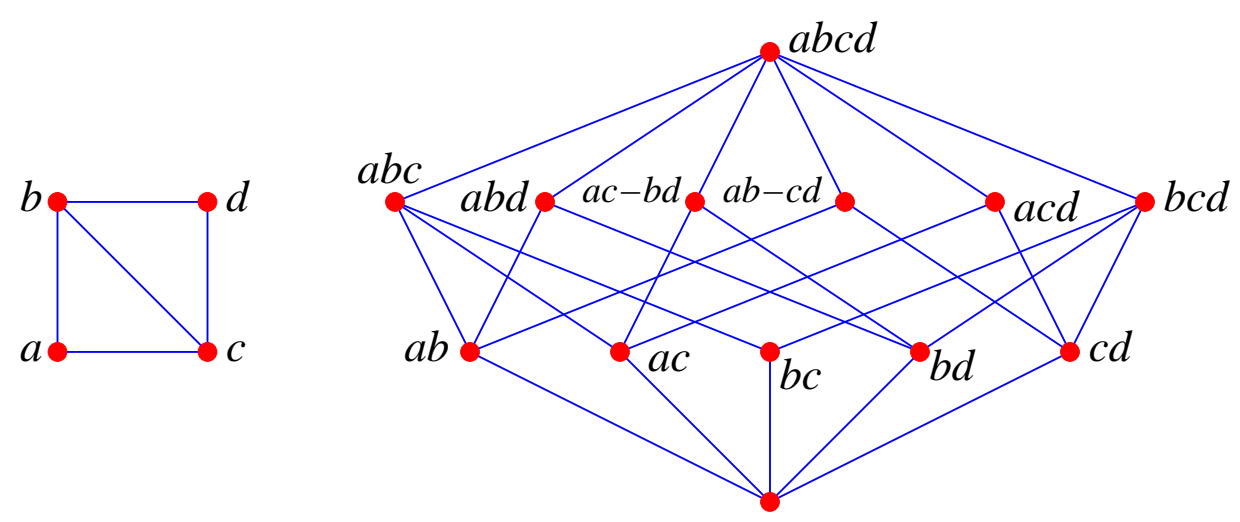

Figure 7. A graph $G$ and its bond lattice $L_{G}$

connected partitions of $V(G)$, i.e., the partitions $\pi=\left\{B_{1}, \ldots, B_{k}\right\}$ of $V(G)=[n]$ such that the restriction of $G$ to each block $B_{i}$ is connected. Namely,

$$
X_{\pi}=\left\{\left(x_{1}, \ldots, x_{n}\right) \in K^{n}: i, j \in B_{m} \text { for some } m \Rightarrow x_{i}=x_{j}\right\} .
$$

We have $X_{\pi} \leq X_{\sigma}$ in $L(\mathcal{A})$ if and only if every block of $\pi$ is contained in a block of $\sigma$. In other words, $\pi$ is a refinement of $\sigma$. This refinement order is the "standard" ordering on $\Pi_{n}$, so $L\left(\mathcal{A}_{G}\right)$ is isomorphic to an induced subposet $L_{G}$ of $\Pi_{n}$, called the bond lattice or lattice of contractions of $G$. ("Induced" means that if $\pi \leq \sigma$ in $\Pi_{n}$ and $\pi, \sigma \in L\left(\mathcal{A}_{G}\right)$, then $\pi \leq \sigma$ in $L\left(\mathcal{A}_{G}\right)$.) In particular, $\Pi_{n} \cong L\left(\mathcal{A}_{K_{n}}\right)$. Note that in general $L_{G}$ is not a sublattice of $\Pi_{n}$, but only a sub-join-semilattice of $\Pi_{n}$ [why?]. The bottom element $\hat{0}$ of $L_{G}$ is the partition of $[n]$ into $n$ one-element blocks, while the top element $\hat{1}$ is the partition into one block. The case $G=K_{n}$ shows that the intersection lattice $L\left(\mathcal{B}_{n}\right)$ of the braid arrangement $\mathcal{B}_{n}$ is isomorphic to the full partition lattice $\Pi_{n}$. Figure 7 shows a graph $G$ and its bond lattice $L_{G}$ (singleton blocks are omitted from the labels of the elements of $L_{G}$ ).

Second proof of Theorem 2.7. Let $\pi \in L_{G}$. For $q \in \mathbb{P}$ define $\chi_{\pi}(q)$ to be the number of colorings $\kappa:[n] \rightarrow[q]$ of $G$ satisfying:

- If $i, j$ are in the same block of $\pi$, then $\kappa(i)=\kappa(j)$.

- If $i, j$ are in different blocks of $\pi$ and $i j \in E(G)$, then $\kappa(i) \neq \kappa(j)$.

Given any $\kappa:[n] \rightarrow[q]$, there is a unique $\sigma \in L_{G}$ such that $\kappa$ is enumerated by $\chi_{\sigma}(q)$. Moreover, $\kappa$ will be constant on the blocks of some $\pi \in L_{G}$ if and only if $\sigma \geq \pi$ in $L_{G}$. Hence

$$
q^{|\pi|}=\sum_{\sigma \geq \pi} \chi_{\sigma}(q) \quad \forall \pi \in L_{G},
$$

where $|\pi|$ denotes the number of blocks of $\pi$. By Möbius inversion,

$$
\chi_{\pi}(q)=\sum_{\sigma \geq \pi} q^{|\sigma|} \mu(\pi, \sigma),
$$

where $\mu$ denotes the Möbius function of $L_{G}$. Let $\pi=\hat{0}$. We get

$$
\chi_{G}(q)=\chi_{\hat{0}}(q)=\sum_{\sigma \in L_{G}} \mu(\sigma) q^{|\sigma|} .
$$


It is easily seen that $|\sigma|=\operatorname{dim} X_{\sigma}$, so comparing equation (21) with Definition 1.3 shows that $\chi_{G}(t)=\chi_{\mathcal{A}_{G}}(t)$.

Corollary 2.2. The characteristic polynomial of the braid arrangement $\mathcal{B}_{n}$ is given by

$$
\chi_{\mathcal{B}_{n}}(t)=t(t-1) \cdots(t-n+1) .
$$

Proof. Since $\mathcal{B}_{n}=\mathcal{A}_{K_{n}}$ (the graphical arrangement of the complete graph $K_{n}$ ), we have from Theorem 2.7 that $\chi_{\mathcal{B}_{n}}(t)=\chi_{K_{n}}(t)$. The proof follows from equation (17).

There is a further invariant of a graph $G$ that is closely connected with the graphical arrangement $\mathcal{A}_{G}$.

Definition 2.7. An orientation o of a graph $G$ is an assignment of a direction $i \rightarrow j$ or $j \rightarrow i$ to each edge $i j$ of $G$. A directed cycle of $\mathfrak{o}$ is a sequence of vertices $i_{0}, i_{1}, \ldots, i_{k}$ of $G$ such that $i_{0} \rightarrow i_{1} \rightarrow i_{2} \rightarrow \cdots \rightarrow i_{k} \rightarrow i_{0}$ in $\mathfrak{o}$. An orientation $\mathfrak{o}$ is acyclic if it contains no directed cycles.

A graph $G$ with no loops (edges from a vertex to itself) thus has $2^{\# E(G)}$ orientations. Let $R \in \mathcal{R}\left(\mathcal{A}_{G}\right)$, and let $\left(x_{1}, \ldots, x_{n}\right) \in R$. In choosing $R$, we have specified for all $i j \in E(G)$ whether $x_{i}<x_{j}$ or $x_{i}>x_{j}$. Indicate by an arrow $i \rightarrow j$ that $x_{i}<x_{j}$, and by $j \rightarrow i$ that $x_{i}>x_{j}$. In this way the region $R$ defines an orientation $\mathfrak{o}_{R}$ of $G$. Clearly if $R \neq R^{\prime}$, then $\mathfrak{o}_{R} \neq \mathfrak{o}_{R^{\prime}}$. Which orientations can arise in this way?

Proposition 2.5. Let $\mathfrak{o}$ be an orientation of $G$. Then $\mathfrak{o}=\mathfrak{o}_{R}$ for some $R \in \mathcal{R}\left(\mathcal{A}_{G}\right)$ if and only if $\mathfrak{o}$ is acyclic.

Proof. If $\mathfrak{o}_{R}$ had a cycle $i_{1} \rightarrow i_{2} \rightarrow \cdots \rightarrow i_{k} \rightarrow i_{1}$, then a point $\left(x_{1}, \ldots, x_{n}\right) \in R$ would satisfy $x_{i_{1}}<x_{i_{2}}<\cdots<x_{i_{k}}<x_{i_{1}}$, which is absurd. Hence $\mathfrak{o}_{R}$ is acyclic.

Conversely, let $\mathfrak{o}$ be an acyclic orientation of $G$. First note that $\mathfrak{o}$ must have a sink, i.e., a vertex with no arrows pointing out. To see this, walk along the edges of $\mathfrak{o}$ by starting at any vertex and following arrows. Since $\mathfrak{o}$ is acyclic, we can never return to a vertex so the process will end in a sink. Let $j_{n}$ be a sink vertex of $\mathfrak{o}$. When we remove $j_{n}$ from $\mathfrak{o}$ the remaining orientation is still acyclic, so it contains a sink $j_{n-1}$. Continuing in this manner, we obtain an ordering $j_{1}, j_{2}, \ldots, j_{n}$ of $[n]$ such that $j_{i}$ is a sink of the restriction of $\mathfrak{o}$ to $j_{1}, \ldots, j_{i}$. Hence if $x_{1}, \ldots, x_{n} \in \mathbb{R}$ satisfy $x_{j_{1}}<x_{j_{2}}<\cdots<x_{j_{n}}$ then the region $R \in \mathcal{R}(\mathcal{A})$ containing $\left(x_{1}, \ldots, x_{n}\right)$ satisfies $\mathfrak{o}=\mathfrak{o}_{R}$.

Note. The transitive, reflexive closure $\overline{\mathfrak{o}}$ of an acyclic orientation $\mathfrak{o}$ is a partial order. The construction of the ordering $j_{1}, j_{2}, \ldots, j_{n}$ above is equivalent to constructing a linear extension of $\mathfrak{o}$.

Let $\mathrm{AO}(G)$ denote the set of acyclic orientations of $G$. We have constructed a bijection between $\mathrm{AO}(G)$ and $\mathcal{R}\left(\mathcal{A}_{G}\right)$. Hence from Theorem 2.5 we conclude:

Corollary 2.3. For any graph $G$ with $n$ vertices, we have $\# A O(G)=(-1)^{n} \chi_{G}(-1)$.

Corollary 2.3 was first proved by Stanley in 1973 by a "direct" argument based on deletion-contraction (see Exercise 7). The proof we have just given based on arrangements is due to Greene and Zaslavsky in 1983.

Note. Given a graph $G$ on $n$ vertices, let $\mathcal{A}_{G}^{\#}$ be the arrangement defined by

$$
x_{i}-x_{j}=a_{i j}, \quad i j \in E(G),
$$


where the $a_{i j}$ 's are generic. Just as we obtained equation (14) (the case $G=K_{n}$ ) we have

$$
\chi_{\mathcal{A}_{G}^{\#}}(t)=\sum_{F}(-1)^{e(F)} t^{n-e(F)},
$$

where $F$ ranges over all spanning forests of $G$. 


\section{Exercises}

(1) [3-] Show that for any arrangement $\mathcal{A}$, we have $\chi_{c \mathcal{A}}(t)=(t-1) \chi_{\mathcal{A}}(t)$, where $c \mathcal{A}$ denotes the cone over $\mathcal{A}$. (Use Whitney's theorem.)

(2) [2-] Let $G$ be a graph on the vertex set $[n]$. Show that the bond lattice $L_{G}$ is a sub-join-semilattice of the partition lattice $\Pi_{n}$ but is not in general a sublattice of $\Pi_{n}$.

(3) [2-] Let $G$ be a forest (graph with no cycles) on the vertex set $[n]$. Show that $L_{G} \cong B_{E(G)}$, the boolean algebra of all subsets of $E(G)$.

(4) [2] Let $G$ be a graph with $n$ vertices and $\mathcal{A}_{G}$ the corresponding graphical arrangement. Suppose that $G$ has a $k$-element clique, i.e., $k$ vertices such that any two are adjacent. Show that $k ! \mid r(\mathcal{A})$.

(5) $[2+]$ Let $G$ be a graph on the vertex set $[n]=\{1,2, \ldots, n\}$, and let $\mathcal{A}_{G}$ be the corresponding graphical arrangement (over any field $K$, but you may assume $K=\mathbb{R}$ if you wish). Let $\mathcal{C}_{n}$ be the coordinate hyperplane arrangement, consisting of the hyperplanes $x_{i}=0,1 \leq i \leq n$. Express $\chi_{\mathcal{A}_{G} \cup e_{n}}(t)$ in terms of $\chi_{\mathcal{A}_{G}}(t)$.

(6) [4] Let $G$ be a planar graph, i.e., $G$ can be drawn in the plane without crossing edges. Show that $\chi_{\mathcal{A}_{G}}(4) \neq 0$.

(7) $[2+]$ Let $G$ be a graph with $n$ vertices. Show directly from the the deletioncontraction recurrence $(20)$ that

$$
(-1)^{n} \chi_{G}(-1)=\# \mathrm{AO}(G) \text {. }
$$

(8) $[2+]$ Let $\chi_{G}(t)=t^{n}-c_{n-1} t^{n-1}+\cdots+(-1)^{n-1} c_{1} t$ be the chromatic polynomial of the graph $G$. Let $i$ be a vertex of $G$. Show that $c_{1}$ is equal to the number of acyclic orientations of $G$ whose unique source is $i$. (A source is a vertex with no arrows pointing in. In particular, an isolated vertex is a source.)

(9) [5] Let $\mathcal{A}$ be an arrangement with characteristic polynomial $\chi_{\mathcal{A}}(t)=t^{n}-$ $c_{n-1} t^{n-1}+c_{n-2} t^{n-2}-\cdots+(-1)^{n} c_{0}$. Show that the sequence $c_{0}, c_{1}, \ldots, c_{n}=1$ is unimodal, i.e., for some $j$ we have

$$
c_{0} \leq c_{1} \leq \cdots \leq c_{j} \geq c_{j+1} \geq \cdots \geq c_{n} .
$$

(10) $[2+]$ Let $f(n)$ be the total number of faces of the braid arrangement $\mathcal{B}_{n}$. Find a simple formula for the generating function

$$
\sum_{n \geq 0} f(n) \frac{x^{n}}{n !}=1+x+3 \frac{x^{2}}{2 !}+13 \frac{x^{3}}{3 !}+75 \frac{x^{4}}{4 !}+541 \frac{x^{5}}{5 !}+4683 \frac{x^{6}}{6 !}+\cdots .
$$

More generally, let $f_{k}(n)$ denote the number of $k$-dimensional faces of $\mathcal{B}_{n}$. For instance, $f_{1}(n)=1$ (for $n \geq 1$ ) and $f_{n}(n)=n$ !. Find a simple formula for the generating function

$$
\sum_{n \geq 0} \sum_{k \geq 0} f_{k}(n) y^{k} \frac{x^{n}}{n !}=1+y x+\left(y+2 y^{2}\right) \frac{x^{2}}{2 !}+\left(y+6 y^{2}+6 y^{3}\right) \frac{x^{3}}{3 !}+\cdots .
$$




\section{LECTURE 3 Matroids and geometric lattices}

\subsection{Matroids}

A matroid is an abstraction of a set of vectors in a vector space (for us, the normals to the hyperplanes in an arrangement). Many basic facts about arrangements (especially linear arrangements) and their intersection posets are best understood from the more general viewpoint of matroid theory. There are many equivalent ways to define matroids. We will define them in terms of independent sets, which are an abstraction of linearly independent sets. For any set $S$ we write

$$
2^{S}=\{T: T \subseteq S\}
$$

Definition 3.8. A (finite) matroid is a pair $M=(S, \mathcal{J})$, where $S$ is a finite set and $\mathcal{J}$ is a collection of subsets of $S$, satisfying the following axioms:

(1) $\mathcal{J}$ is a nonempty (abstract) simplicial complex, i.e., $\mathcal{J} \neq \emptyset$, and if $J \in \mathcal{J}$ and $I \subset J$, then $I \in \mathcal{J}$.

(2) For all $T \subseteq S$, the maximal elements of $\mathcal{J} \cap 2^{T}$ have the same cardinality. In the language of simplicial complexes, every induced subcomplex of $\mathcal{J}$ is pure.

The elements of $\mathcal{J}$ are called independent sets. All matroids considered here will be assumed to be finite. By standard abuse of notation, if $M=(S, \mathcal{J})$ then we write $x \in M$ to mean $x \in S$. The archetypal example of a matroid is a finite subset $S$ of a vector space, where independence means linear independence. A closely related matroid consists of a finite subset $S$ of an affine space, where independence now means affine independence.

It should be clear what is meant for two matroids $M=(S, \mathcal{J})$ and $M^{\prime}=\left(S^{\prime}, \mathcal{J}^{\prime}\right)$ to be isomorphic, viz., there exists a bijection $f: S \rightarrow S^{\prime}$ such that $\left\{x_{1}, \ldots, x_{j}\right\} \in \mathcal{J}$ if and only if $\left\{f\left(x_{1}\right), \ldots, f\left(x_{j}\right)\right\} \in \mathcal{J}^{\prime}$. Let $M$ be a matroid and $S$ a set of points in $\mathbb{R}^{n}$, regarded as a matroid with independence meaning affine independence. If $M$ and $S$ are isomorphic matroids, then $S$ is called an affine diagram of $M$. (Not all matroids have affine diagrams.)

Example 3.7. (a) Regard the configuration in Figure 1 as a set of five points in the two-dimensional affine space $\mathbb{R}^{2}$. These five points thus define the affine diagram of a matroid $M$. The lines indicate that the points $1,2,3$ and $3,4,5$ lie on straight 


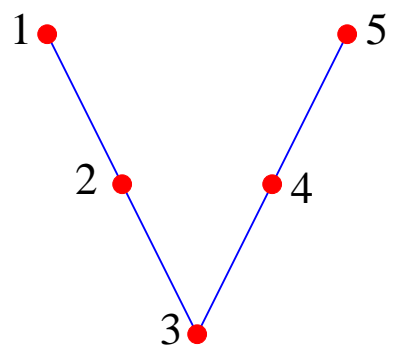

Figure 1. A five-point matroid in the affine space $\mathbb{R}^{2}$

lines. Hence the sets $\{1,2,3\}$ and $\{3,4,5\}$ are affinely dependent in $\mathbb{R}^{2}$ and therefore dependent (i.e., not independent) in $M$. The independent sets of $M$ consist of all subsets of [5] with at most two elements, together with all three-element subsets of [5] except 123 and 345 (where 123 is short for $\{1,2,3\}$, etc.). i.e.,

(b) Write $\mathcal{J}=\left\langle S_{1}, \ldots, S_{k}\right\rangle$ for the simplicial complex J generated by $S_{1}, \ldots, S_{k}$,

$$
\begin{aligned}
\left\langle S_{1}, \ldots, S_{k}\right\rangle & =\left\{T: T \subseteq S_{i} \text { for some } i\right\} \\
& =2^{S_{1}} \cup \cdots \cup 2^{S_{k}} .
\end{aligned}
$$

Then $\mathcal{J}=\langle 13,14,23,24\rangle$ is the set of independent sets of a matroid $M$ on [4]. This matroid is realized by a multiset of vectors in a vector space or affine space, e.g., by the points $1,1,2,2$ in the affine space $\mathbb{R}$. The affine diagam of this matroid is given by

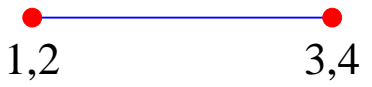

(c) Let $\mathcal{J}=\langle 12,23,34,45,15\rangle$. Then $\mathcal{J}$ is not the set of independent sets of a matroid. For instance, the maximal elements of $\mathcal{J} \cap 2^{\{1,2,4\}}$ are 12 and 4 , which do not have the same cardinality.

(d) The affine diagram below shows a seven point matroid.

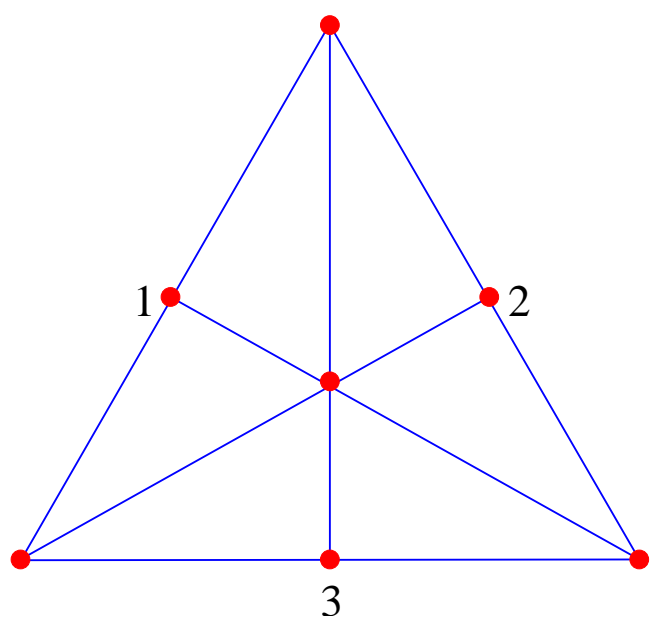


If we further require the points labelled $1,2,3$ to lie on a line (i.e., remove 123 from J), we still have a matroid $M$, but not one that can be realized by real vectors. In fact, $M$ is isomorphic to the set of nonzero vectors in the vector space $\mathbb{F}_{2}^{3}$, where $\mathbb{F}_{2}$ denotes the two-element field.

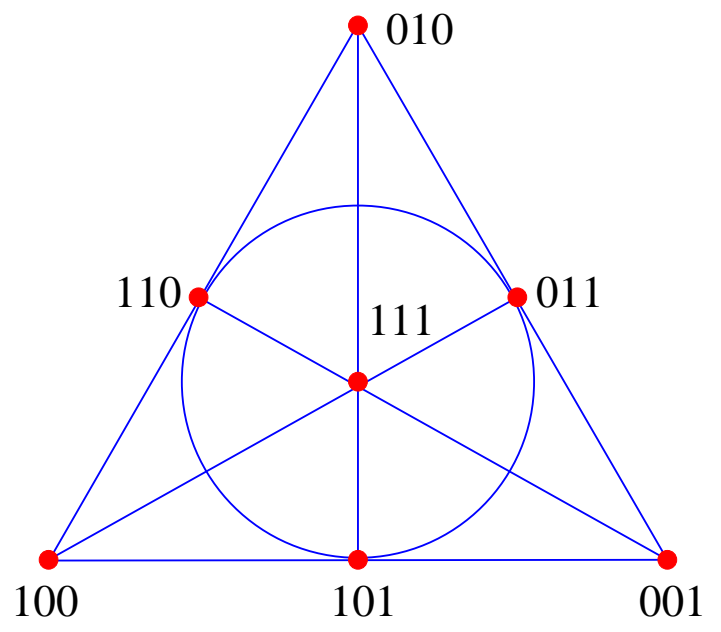

Let us now define a number of important terms associated to a matroid $M$. A basis of $M$ is a maximal independent set. A circuit $C$ is a minimal dependent set, i.e., $C$ is not independent but becomes independent when we remove any point from it. For example, the circuits of the matroid of Figure 1 are 123, 345, and 1245.

If $M=(S, \mathcal{J})$ is a matroid and $T \subseteq S$ then define the rank $\operatorname{rk}(T)$ of $T$ by

$$
\operatorname{rk}(T)=\max \{\# I: I \in \mathcal{J} \text { and } I \subseteq T\} .
$$

In particular, $\operatorname{rk}(\emptyset)=0$. We define the rank of the matroid $M$ itself by $\operatorname{rk}(M)=$ $\operatorname{rk}(S)$. A $k$-flat is a maximal subset of rank $k$. For instance, if $M$ is an affine matroid, i.e., if $S$ is a subset of an affine space and independence in $M$ is given by affine independence, then the flats of $M$ are just the intersections of $S$ with affine subspaces. Note that if $F$ and $F^{\prime}$ are flats of a matroid $M$, then so is $F \cap F^{\prime}$ (see Exercise 2). Since the intersection of flats is a flat, we can define the closure $\bar{T}$ of a subset $T \subseteq S$ to be the smallest flat containing $T$, i.e.,

$$
\bar{T}=\bigcap_{\text {flats } F \supseteq T} F .
$$

This closure operator has a number of nice properties, such as $\overline{\bar{T}}=\bar{T}$ and $T^{\prime} \subseteq$ $T \Rightarrow \bar{T}^{\prime} \subseteq \bar{T}$.

\subsection{The lattice of flats and geometric lattices}

For a matroid $M$ define $L(M)$ to be the poset of flats of $M$, ordered by inclusion. Since the intersection of flats is a flat, $L(M)$ is a meet-semilattice; and since $L(M)$ has a top element $S$, it follows from Lemma 2.3 that $L(M)$ is a lattice, which we call the lattice of flats of $M$. Note that $L(M)$ has a unique minimal element $\hat{0}$, viz., $\bar{\emptyset}$ or equivalently, the intersection of all flats. It is easy to see that $L(M)$ is graded by rank, i.e., every maximal chain of $L(M)$ has length $m=\operatorname{rk}(M)$. Thus if $x \lessdot y$ in 


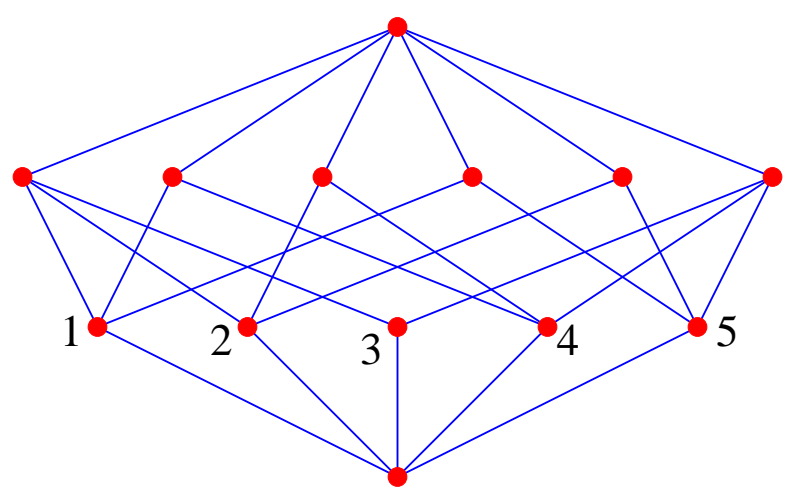

Figure 2. The lattice of flats of the matroid of Figure 1

$L(M)$ then $\operatorname{rk}(y)=1+\operatorname{rk}(x)$. We now define the characteristic polynomial $\chi_{M}(t)$, in analogy to the definition (3) of $\chi_{\mathcal{A}}(t)$, by

$$
\chi_{M}(t)=\sum_{x \in L(M)} \mu(\hat{0}, x) t^{m-\operatorname{rk}(x)},
$$

where $\mu$ denotes the Möbius function of $L(M)$ and $m=\operatorname{rk}(M)$. Figure 2 shows the lattice of flats of the matroid $M$ of Figure 1. From this figure we see easily that

$$
\chi_{M}(t)=t^{3}-5 t^{2}+8 t-4 .
$$

Let $M$ be a matroid and $x \in M$. If the set $\{x\}$ is dependent (i.e., if $\operatorname{rk}(\{x\})=0$ ) then we call $x$ a loop. Thus $\bar{\emptyset}$ is just the set of loops of $M$. Suppose that $x, y \in M$, neither $x$ nor $y$ are loops, and $\operatorname{rk}(\{x, y\})=1$. We then call $x$ and $y$ parallel points. A matroid is simple if it has no loops or pairs of parallel points. It is clear that the following three conditions are equivalent:

- $M$ is simple.

- $\bar{\emptyset}=\emptyset$ and $\bar{x}=x$ for all $x \in M$.

- $\operatorname{rk}(\{x, y\})=2$ for all points $x \neq y$ of $M$ (assuming $M$ has at least two points).

For any matroid $M$ and $x, y \in M$, define $x \sim y$ if $\bar{x}=\bar{y}$. It is easy to see that $\sim$ is an equivalence relation. Let

$$
\widehat{M}=\{\bar{x}: x \in M, x \notin \bar{\emptyset}\},
$$

with an obvious definition of independence, i.e.,

$$
\left\{\bar{x}_{1}, \ldots, \bar{x}_{k}\right\} \in \mathcal{J}(\widehat{M}) \Leftrightarrow\left\{x_{1}, \ldots, x_{k}\right\} \in \mathcal{J}(M) .
$$

Then $\widehat{M}$ is simple, and $L(M) \cong L(\widehat{M})$. Thus insofar as intersection lattices $L(M)$ are concerned, we may assume that $M$ is simple. (Readers familiar with point set topology will recognize the similarity between the conditions for a matroid to be simple and for a topological space to be $T_{0}$.)

Example 3.8. Let $S$ be any finite set and $V$ a vector space. If $f: S \rightarrow V$, then define a matroid $M_{f}$ on $S$ by the condition that given $I \subseteq S$,

$$
I \in \mathcal{J}(M) \Leftrightarrow\{f(x): x \in I\} \text { is linearly independent. }
$$


Then a loop is any element $x$ satisfying $f(x)=0$, and $x \sim y$ if and only if $f(x)$ is a nonzero scalar multiple of $f(y)$.

Note. If $M=(S, \mathcal{J})$ is simple, then $L(M)$ determines $M$. For we can identify $S$ with the set of atoms of $L(M)$, and we have

$$
\left\{x_{1}, \ldots, x_{k}\right\} \in \mathcal{J} \Leftrightarrow \operatorname{rk}\left(x_{1} \vee \cdots \vee x_{k}\right)=k \text { in } L(M) .
$$

See the proof of Theorem 3.8 for further details.

We now come to the primary connection between hyperplane arrangements and matroid theory. If $H$ is a hyperplane, write $\mathfrak{n}_{H}$ for some (nonzero) normal vector to $H$.

Proposition 3.6. Let $\mathcal{A}$ be a central arrangement in the vector space $V$. Define a matroid $M=M_{\mathcal{A}}$ on $\mathcal{A}$ by letting $\mathcal{B} \in \mathcal{J}(M)$ if $\mathcal{B}$ is linearly independent (i.e., $\left\{\mathfrak{n}_{H}: H \in \mathcal{B}\right\}$ is linearly independent). Then $M$ is simple and $L(M) \cong L(\mathcal{A})$.

Proof. $M$ has no loops, since every $H \in \mathcal{A}$ has a nonzero normal. Two distinct nonparallel hyperplanes have linearly independent normals, so the points of $M$ are closed. Hence $M$ is simple.

Let $\mathcal{B}, \mathcal{B}^{\prime} \subseteq \mathcal{A}$, and set

$$
X=\bigcap_{H \in \mathcal{B}} H=X_{\mathcal{B}}, \quad X^{\prime}=\bigcap_{H \in \mathcal{B}^{\prime}} H=X_{\mathcal{B}^{\prime}} .
$$

Then $X=X^{\prime}$ if and only if

$$
\operatorname{span}\left\{\mathfrak{n}_{H}: H \in \mathcal{B}\right\}=\operatorname{span}\left\{\mathfrak{n}_{H}: H \in \mathcal{B}^{\prime}\right\} .
$$

Now the closure relation in $M$ is given by

$$
\overline{\mathcal{B}}=\left\{H^{\prime} \in \mathcal{A}: \mathfrak{n}_{H^{\prime}} \in \operatorname{span}\left\{\mathfrak{n}_{H}: H \in \mathcal{B}\right\}\right\} .
$$

Hence $X=X^{\prime}$ if and only if $\bar{B}=\bar{B}^{\prime}$, so $L(M) \cong L(\mathcal{A})$.

It follows that for a central arrangement $\mathcal{A}, L(\mathcal{A})$ depends only on the matroidal structure of $\mathcal{A}$, i.e., which subsets of hyperplanes are linearly independent. Thus the matroid $M_{\mathcal{A}}$ encapsulates the essential information about $\mathcal{A}$ needed to define $L(\mathcal{A})$.

Our next goal is to characterize those lattices $L$ which have the form $L(M)$ for some matroid $M$.

Proposition 3.7. Let $L$ be a finite graded lattice. The following two conditions are equivalent.

(1) For all $x, y \in L$, we have $\operatorname{rk}(x)+\operatorname{rk}(y) \geq \operatorname{rk}(x \wedge y)+\operatorname{rk}(x \vee y)$.

(2) If $x$ and $y$ both cover $x \wedge y$, then $x \vee y$ covers both $x$ and $y$.

Proof. Assume (1). Let $x, y>x \wedge y$, so $\operatorname{rk}(x)=\operatorname{rk}(y)=\operatorname{rk}(x \wedge y)+1$ and $\operatorname{rk}(x \vee y)>\operatorname{rk}(x)=\operatorname{rk}(y)$. By (1),

$$
\begin{aligned}
\operatorname{rk}(x)+\operatorname{rk}(y) & \geq(\operatorname{rk}(x)-1)+\operatorname{rk}(x \vee y) \\
\Rightarrow \operatorname{rk}(y) & \geq \operatorname{rk}(x \vee y)-1 \\
\Rightarrow x \vee y & >x .
\end{aligned}
$$

Similarly $x \vee y>y$, proving (2).

For $(2) \Rightarrow(1)$, see [31, Prop. 3.3.2]. 


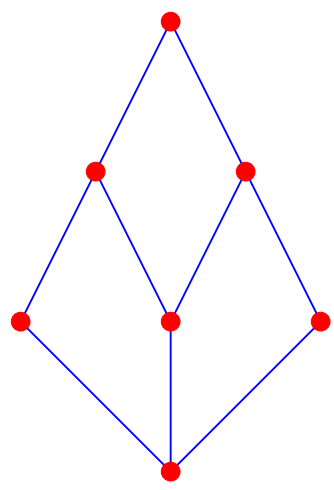

(a)

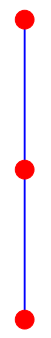

(b)

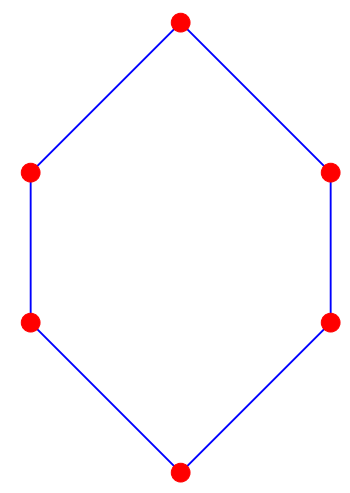

(c)

Figure 3. Three nongeometric lattices

Definition 3.9. A finite lattice $L$ satisfying condition (1) or (2) above is called (upper) semimodular. A finite lattice $L$ is atomic if every $x \in L$ is a join of atoms (where we regard $\hat{0}$ as an empty join of atoms). Equivalently, if $x \in L$ is joinirreducible (i.e., covers a unique element), then $x$ is an atom. Finally, a finite lattice is geometric if it is both semimodular and atomic.

To illustrate these definitions, Figure 3(a) shows an atomic lattice that is not semimodular, (b) shows a semimodular lattice that is not atomic, and (c) shows a graded lattice that is neither semimodular nor atomic.

We are now ready to characterize the lattice of flats of a matroid.

Theorem 3.8. Let $L$ be a finite lattice. The following two conditions are equivalent.

(1) $L$ is a geometric lattice.

(2) $L \cong L(M)$ for some (simple) matroid $M$.

Proof. Assume that $L$ is geometric, and let $A$ be the set of atoms of $L$. If $T \subseteq A$ then write $\bigvee T=\bigvee_{x \in T} x$, the join of all elements of $T$. Let

$$
\mathcal{J}=\{I \subseteq A: \operatorname{rk}(\vee I)=\# I\} .
$$

Note that by semimodularity, we have for any $S \subseteq A$ and $x \in A$ that $\operatorname{rk}((\bigvee S) \vee x) \leq$ $\operatorname{rk}(\bigvee S)+1$. (Hence in particular, $\operatorname{rk}(\bigvee S) \leq \# S$.) It follows that $\mathcal{J}$ is a simplicial complex. Let $S \subseteq A$, and let $T, T^{\prime}$ be maximal elements of $2^{S} \cap \mathcal{J}$. We need to show that $\# T=\# T^{\prime}$.

Assume $\# T<\# T^{\prime}$, say. If $y \in S$ then $y \leq \bigvee T^{\prime}$, else $T^{\prime \prime}=T^{\prime} \cup y$ satisfies $\operatorname{rk}\left(\bigvee T^{\prime \prime}\right)=\# T^{\prime \prime}$, contradicting the maximality of $T^{\prime}$. Since $\# T<\# T^{\prime}$ and $T \subseteq S$, it follows that $\bigvee T<\bigvee T^{\prime}$ [why?]. Since $L$ is atomic, there exists $y \in S$ such that $y \in S$ but $y \not \leq \bigvee T$. But then $\operatorname{rk}(\bigvee(T \cup y))=1+\# T$, contradicting the maximality of $T$. Hence $M=(A, \mathcal{J})$ is a matroid, and $L \cong L(M)$.

Conversely, given a matroid $M$, which we may assume is simple, we need to show that $L(M)$ is a geometric lattice. Clearly $L(M)$ is atomic, since every flat is the join of its elements. Let $S, T \subseteq M$. We will show that

$$
\operatorname{rk}(S)+\operatorname{rk}(T) \geq \operatorname{rk}(S \cap T)+\operatorname{rk}(S \cup T) .
$$


Note that if $S$ and $T$ are flats (i.e., $S, T \in L(M)$ ) then $S \cap T=S \wedge T$ and $\operatorname{rk}(S \cup T)=\operatorname{rk}(S \vee T)$. Hence taking $S$ and $T$ to be flats in (24) shows that $L(M)$ is semimodular and thus geometric. Suppose (24) is false, so

$$
\operatorname{rk}(S \cup T)>\operatorname{rk}(S)+\operatorname{rk}(T)-\operatorname{rk}(S \cap T) .
$$

Let $B$ be a basis for $S \cup T$ extending a basis for $S \cup T$. Then either $\#(B \cap S)>\operatorname{rk}(S)$ or $\#(B \cap T)>\operatorname{rk}(T)$, a contradiction completing the proof.

Note that by Proposition 3.6 and Theorem 3.8, any results we prove about geometric lattices hold a fortiori for the intersection lattice $L_{\mathcal{A}}$ of a central arrangement $\mathcal{A}$.

Note. If $L$ is geometric and $x \leq y$ in $L$, then it is easy to show using semimodularity that the interval $[x, y]$ is also a geometric lattice. (See Exercise 3.) In general, however, an interval of an atomic lattice need not be atomic.

For noncentral arrangements $L(\mathcal{A})$ is not a lattice, but there is still a connection with geometric lattices. For a stronger statement, see Exercise 4.

Proposition 3.8. Let $\mathcal{A}$ be an arrangement. Then every interval $[x, y]$ of $L(\mathcal{A})$ is a geometric lattice.

Proof. By Exercise 3, it suffices to take $x=\hat{0}$. Now $[\hat{0}, y] \cong L\left(\mathcal{A}_{y}\right)$, where $\mathcal{A}_{y}$ is given by (6). Since $\mathcal{A}_{y}$ is a central arrangement, the proof follows from Proposition 3.6 .

The proof of our next result about geometric lattices will use a fundamental formula concerning Möbius functions known as Weisner's theorem. For a proof, see [31, Cor. 3.9.3] (where it is stated in dual form).

Theorem 3.9. Let $L$ be a finite lattice with at least two elements and with Möbius function $\mu$. Let $\hat{0} \neq a \in L$. Then

$$
\sum_{x: x \vee a=\hat{1}} \mu(x)=0 .
$$

Note that Theorem 3.9 gives a "shortening" of the recurrence (2) defining $\mu$. Normally we take $a$ to be an atom, since that produces fewer terms in (25) than choosing any $b>a$. As an example, let $L=B_{n}$, the boolean algebra of all subsets of $[n]$, and let $a=\{n\}$. There are two elements $x \in B_{n}$ such that $x \vee a=\hat{1}=[n]$, viz., $x_{1}=[n-1]$ and $x_{2}=[n]$. Hence $\mu\left(x_{1}\right)+\mu\left(x_{2}\right)=0$. Since $\left[\hat{0}, x_{1}\right]=B_{n-1}$ and $\left[\hat{0}, x_{2}\right]=B_{n}$, we easily obtain $\mu_{B_{n}}(\hat{1})=(-1)^{n}$, agreeing with (4).

If $x \leq y$ in a graded lattice $L$, write $\operatorname{rk}(x, y)=\operatorname{rk}(y)-\operatorname{rk}(x)$, the length of every saturated chain from $x$ to $y$. The next result may be stated as "the Möbius function of a geometric lattice strictly alternates in sign."

Theorem 3.10. Let $L$ be a finite geometric lattice with Möbius function $\mu$, and let $x \leq y$ in L. Then

$$
(-1)^{\mathrm{rk}(x, y)} \mu(x, y)>0 .
$$

Proof. Since every interval of a geometric lattice is a geometric lattice (Exercise 3), it suffices to prove the theorem for $[x, y]=[\hat{0}, \hat{1}]$. The proof is by induction on the rank of $L$. It is clear if $\operatorname{rk}(L)=1$, in which case $\mu(\hat{0}, \hat{1})=-1$. Assume the result for geometric lattices of $\operatorname{rank}<n$, and $\operatorname{let} \operatorname{rk}(L)=n$. Let $a$ be an atom of $L$ in Theorem 3.9. For any $y \in L$ we have by semimodularity that

$$
\operatorname{rk}(y \wedge a)+\operatorname{rk}(y \vee a) \leq \operatorname{rk}(y)+\operatorname{rk}(a)=\operatorname{rk}(y)+1 .
$$


Hence $x \vee a=\hat{1}$ if and only if $x=\hat{1}$ or $x$ is a coatom (i.e., $x \lessdot \hat{1}$ ) satisfying $a \not \leq x$. From Theorem 3.9 there follows

$$
\mu(\hat{0}, \hat{1})=-\sum_{a \succeq x<\hat{1}} \mu(\hat{0}, x) .
$$

The sum on the right is nonempty since $L$ is atomic, and by induction every $x$ indexing the sum satisfies $(-1)^{n-1} \mu(\hat{0}, x)>0$. Hence $(-1)^{n} \mu(\hat{0}, \hat{1})>0$.

Combining Proposition 3.8 and Theorem 3.10 yields the following result.

Corollary 3.4. Let $\mathcal{A}$ be any arrangement and $x \leq y$ in $L(\mathcal{A})$. Then

$$
(-1)^{\mathrm{rk}(x, y)} \mu(x, y)>0,
$$

where $\mu$ denotes the Möbius function of $L(\mathcal{A})$.

Similarly, combining Theorem 3.10 with the definition $(22)$ of $\chi_{M}(t)$ gives the next corollary.

Corollary 3.5. Let $M$ be a matroid of rank $n$. Then the characteristic polynomial $\chi_{M}(t)$ strictly alternates in sign, i.e., if

$$
\chi_{M}(t)=a_{n} t^{n}+a_{n-1} t^{n-1}+\cdots+a_{0},
$$

then $(-1)^{n-i} a_{i}>0$ for $0 \leq i \leq n$.

Let $\mathcal{A}$ be an $n$-dimensional arrangement of rank $r$. If $M_{\mathcal{A}}$ is the matroid corresponding to $\mathcal{A}$, as defined in Proposition 3.6, then

$$
\chi_{\mathcal{A}}(t)=t^{n-r} \chi_{M}(t) .
$$

It follows from Corollary 3.5 and equation (26) that we can write

$$
\chi_{\mathcal{A}}(t)=b_{n} t^{n}+b_{n-1} t^{n-1}+\cdots+b_{n-r} t^{n-r},
$$

where $(-1)^{n-i} b_{i}>0$ for $n-r \leq i \leq n$. 


\section{Exercises}

(1) (a) $[1+]$ Let $\chi_{G}(t)$ be the characteristic polynomial of the graphical arrangement $\mathcal{A}_{G}$. Suppose that $\chi_{G}(i)=0$, where $i \in \mathbb{Z}, i>1$. Show that $\chi_{G}(i-1)=0$.

(b) [2] Is the same conclusion true for any central arrangement $\mathcal{A}$ ?

(2) [2] Show that if $F$ and $F^{\prime}$ are flats of a matroid $M$, then so is $F \cap F^{\prime}$.

(3) [2] Prove the assertion in the Note following the proof of Theorem 3.8 that an interval $[x, y]$ of a geometric lattice $L$ is also a geometric lattice.

(4) [2-] Let $\mathcal{A}$ be an arrangement (not necessarily central), and let $c \mathcal{A}$ denote the cone over $\mathcal{A}$. Show that there exists an atom $a$ of $L(c \mathcal{A})$ such that $L(\mathcal{A}) \cong$ $L(c \mathcal{A})-V_{a}$, where $V_{a}=\{x \in L: x \geq a\}$.

(5) [2-] Let $L$ be a geometric lattice of rank $n$, and define the truncation $T(L)$ to be the subposet of $L$ consisting of all elements of rank $\neq n-1$. Show that $T(L)$ is a geometric lattice.

(6) Let $W_{i}$ be the number of elements of rank $i$ in a geometric lattice (or just in the intersection poset of a central hyperplane arrangement, if you prefer) of rank $n$.

(a) [3] Show that for $k \leq n / 2$,

$$
W_{1}+W_{2}+\cdots+W_{k} \leq W_{n-k}+W_{n-k+1}+\cdots+W_{n-1} .
$$

(b) [2-] Deduce from (a) and Exercise 5 that $W_{1} \leq W_{k}$ for all $1 \leq k \leq n-1$.

(c) [5] Show that $W_{i} \leq W_{n-i}$ for $i<n / 2$ and that the sequence $W_{0}, W_{1}, \ldots, W_{n}$ is unimodal. (Compare Lecture 2, Exercise 9.)

(7) [3-] Let $x \leq y$ in a geometric lattice $L$. Show that $\mu(x, y)= \pm 1$ if and only if the interval $[x, y]$ is isomorphic to a boolean algebra. (Use Weisner's theorem.) Note. This problem becomes much easier using Theorem 4.12 (the Broken Circuit Theorem); see Exercise 4.13. 



\section{LECTURE 4 Broken circuits, modular elements, and supersolvability}

This lecture is concerned primarily with matroids and geometric lattices. Since the intersection lattice of a central arrangement is a geometric lattice, all our results can be applied to arrangements.

\subsection{Broken circuits}

For any geometric lattice $L$ and $x \leq y$ in $L$, we have seen (Theorem 3.10) that $(-1)^{\mathrm{rk}(x, y)} \mu(x, y)$ is a positive integer. It is thus natural to ask whether this integer has a direct combinatorial interpretation. To this end, let $M$ be a matroid on the set $S=\left\{u_{1}, \ldots, u_{m}\right\}$. Linearly order the elements of $S$, say $u_{1}<u_{2}<\cdots<u_{m}$. Recall that a circuit of $M$ is a minimal dependent subset of $S$.

Definition 4.10. A broken circuit of $M$ (with respect to the linear ordering $\mathcal{O}$ of $S$ ) is a set $C-\{u\}$, where $C$ is a circuit and $u$ is the largest element of $C$ (in the ordering $\mathcal{O}$ ). The broken circuit complex $\mathrm{BC}_{\mathcal{O}}(M)$ (or just $\mathrm{BC}(M)$ if no confusion will arise) is defined by

$$
\mathrm{BC}(M)=\{T \subseteq S: T \text { contains no broken circuit }\} .
$$

Figure 1 shows two linear orderings $\mathcal{O}$ and $\mathcal{O}^{\prime}$ of the points of the affine matroid $M$ of Figure 1 (where the ordering of the points is $1<2<3<4<5$ ). With respect to the first ordering $\mathcal{O}$ the circuits are 123, 345, 1245, and the broken circuits are $12,34,124$. With respect to the second ordering $\mathcal{O}^{\prime}$ the circuits are $123,145,2345$, and the broken circuits are 12, 14, 234 .

It is clear that the broken circuit complex $\mathrm{BC}(M)$ is an abstract simplicial complex, i.e., if $T \in \mathrm{BC}(M)$ and $U \subseteq T$, then $U \in \mathrm{BC}(M)$. In Figure 1 we
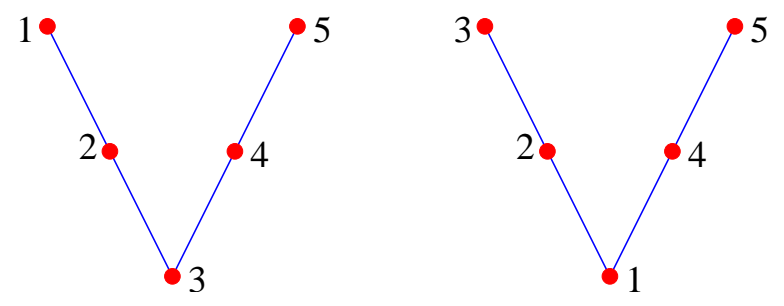

Figure 1. Two linear orderings of the matroid $M$ of Figure 1 
have $\mathrm{BC}_{\mathcal{O}}(M)=\langle 135,145,235,245\rangle$, while $\mathrm{BC}_{\mathcal{O}^{\prime}}(M)=\langle 135,235,245,345\rangle$. These simplicial complexes have geometric realizations as follows:
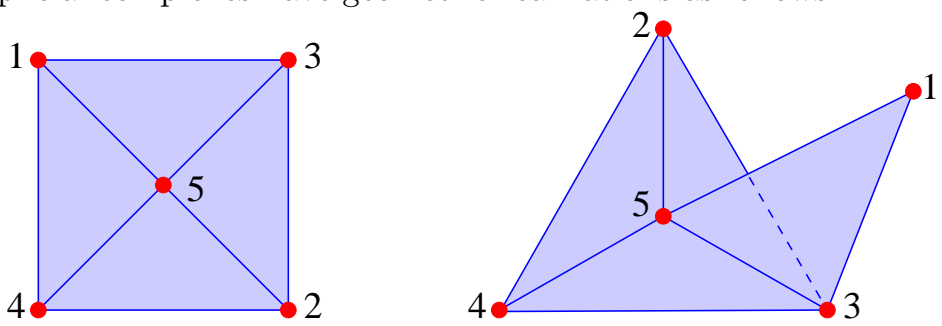

Note that the two simplicial complexes $\mathrm{BC}_{\mathcal{O}}(M)$ and $\mathrm{BC}_{\mathcal{O}^{\prime}}(M)$ are not isomorphic (as abstract simplicial complexes); in fact, their geometric realizations are not even homeomorphic. On the other hand, if $f_{i}(\Delta)$ denotes the number of $i$ dimensional faces (or faces of cardinality $i-1$ ) of the abstract simplicial complex $\Delta$, then for $\Delta$ given by either $\mathrm{BC}_{\mathcal{O}}(M)$ or $\mathrm{BC}_{\mathcal{O}^{\prime}}(M)$ we have

$$
f_{-1}(\Delta)=1, f_{0}(\Delta)=5, f_{1}(\Delta)=8, f_{2}(\Delta)=4 .
$$

Note, moreover, that

$$
\chi_{M}(t)=t^{3}-5 t^{2}+8 t-4 .
$$

In order to generalize this observation to arbitrary matroids, we need to introduce a fair amount of machinery, much of it of interest for its own sake. First we give a fundamental formula, known as Philip Hall's theorem, for the Möbius function value $\mu(\hat{0}, \hat{1})$.

Lemma 4.4. Let $P$ be a finite poset with $\hat{0}$ and $\hat{1}$, and with Möbius function $\mu$. Let $c_{i}$ denote the number of chains $\hat{0}=y_{0}<y_{1}<\cdots<y_{i}=\hat{1}$ in $P$. Then

$$
\mu(\hat{0}, \hat{1})=-c_{1}+c_{2}-c_{3}+\cdots .
$$

Proof. We work in the incidence algebra $\mathcal{J}(P)$. We have

$$
\begin{aligned}
\mu(\hat{0}, \hat{1}) & =\zeta^{-1}(\hat{0}, \hat{1}) \\
& =(\delta+(\zeta-\delta))^{-1}(\hat{0}, \hat{1}) \\
& =\delta(\hat{0}, \hat{1})-(\zeta-\delta)(\hat{0}, \hat{1})+(\zeta-\delta)^{2}(\hat{0}, \hat{1})-\cdots .
\end{aligned}
$$

This expansion is easily justified since $(\zeta-\delta)^{k}(\hat{0}, \hat{1})=0$ if the longest chain of $P$ has length less than $k$. By definition of the product in $\mathcal{J}(P)$ we have $(\zeta-\delta)^{i}(\hat{0}, \hat{1})=c_{i}$, and the proof follows.

Note. Let $P$ be a finite poset with $\hat{0}$ and $\hat{1}$, and let $P^{\prime}=P-\{\hat{0}, \hat{1}\}$. Define $\Delta\left(P^{\prime}\right)$ to be the set of chains of $P^{\prime}$, so $\Delta\left(P^{\prime}\right)$ is an abstract simplicial complex. The reduced Euler characteristic of a simplicial complex $\Delta$ is defined by

$$
\tilde{\chi}(P)=-f_{-1}+f_{0}-f_{1}+\cdots,
$$

where $f_{i}$ is the number of $i$-dimensional faces $F \in \Delta$ (or $\left.\# F=i+1\right)$. Comparing with Lemma 4.4 shows that

$$
\mu(\hat{0}, \hat{1})=\tilde{\chi}\left(\Delta\left(P^{\prime}\right)\right) .
$$

Readers familiar with topology will know that $\tilde{\chi}(\Delta)$ has important topological significance related to the homology of $\Delta$. It is thus natural to ask whether results 


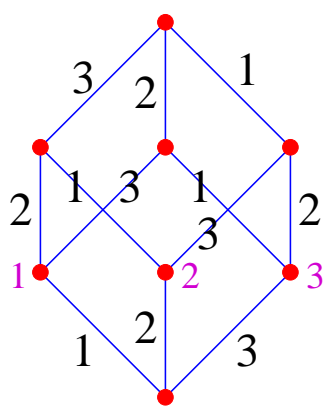

(a)

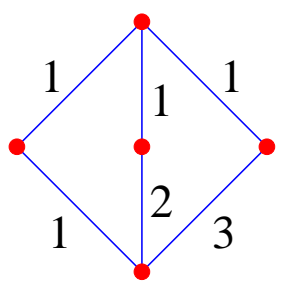

(b)

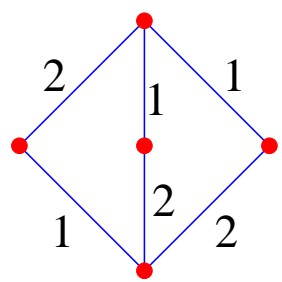

(c)

Figure 2. Three examples of edge-labelings

concerning Möbius functions can be generalized or refined topologically. Such results are part of the subject of "topological combinatorics," about which we will say a little more later.

Now let $P$ be a finite graded poset with $\hat{0}$ and $\hat{1}$. Let

$$
\mathcal{E}(P)=\{(x, y): x \lessdot y \text { in } P\},
$$

the set of (directed) edges of the Hasse diagram of $P$.

Definition 4.11. An E-labeling of $P$ is a map $\lambda: \mathcal{E}(P) \rightarrow \mathbb{P}$ such that if $x<y$ in $P$ then there exists a unique saturated chain

$$
C: x=x_{0} \lessdot x_{1} \lessdot x_{1} \lessdot \cdots \lessdot x_{k}=y
$$

satisfying

$$
\lambda\left(x_{0}, x_{1}\right) \leq \lambda\left(x_{1}, x_{2}\right) \leq \cdots \leq \lambda\left(x_{k-1}, x_{k}\right)
$$

We call $C$ the increasing chain from $x$ to $y$.

Figure 2 shows three examples of posets $P$ with a labeling of their edges, i.e. a map $\lambda: \mathcal{E}(P) \rightarrow \mathbb{P}$. Figure 2(a) is the boolean algebra $B_{3}$ with the labeling $\lambda(S, S \cup\{i\})=i$. (The one-element subsets $\{i\}$ are also labelled with a small $i$.) For any boolean algebra $B_{n}$, this labeling is the archetypal example of an $E$ labeling. The unique increasing chain from $S$ to $T$ is obtained by adjoining to $S$ the elements of $T-S$ one at a time in increasing order. Figures 2(b) and (c) show two different $E$-labelings of the same poset $P$. These labelings have a number of different properties, e.g., the first has a chain whose edge labels are not all different, while every maximal chain label of Figure $2(\mathrm{c})$ is a permutation of $\{1,2\}$.

Theorem 4.11. Let $\lambda$ be an E-labeling of $P$, and let $x \leq y$ in $P$. Let $\mu$ denote the Möbius function of $P$. Then $(-1)^{\mathrm{rk}(x, y)} \mu(x, y)$ is equal to the number of strictly decreasing saturated chains from $x$ to $y$, i.e.,

$$
\begin{aligned}
(-1)^{\mathrm{rk}(x, y)} \mu(x, y) & = \\
\#\left\{x=x_{0} \lessdot x_{1} \lessdot \cdots \lessdot x_{k}\right. & \left.=y: \lambda\left(x_{0}, x_{1}\right)>\lambda\left(x_{1}, x_{2}\right)>\cdots>\lambda\left(x_{k-1}, x_{k}\right)\right\} .
\end{aligned}
$$

Proof. Since $\lambda$ restricted to $[x, y]$ (i.e., to $\mathcal{E}([x, y]))$ is an $E$-labeling, we can assume $[x, y]=[\hat{0}, \hat{1}]=P$. Let $S=\left\{a_{1}, a_{2}, \ldots, a_{j-1}\right\} \subseteq[n-1]$, with $a_{1}<a_{2}<\cdots<a_{j-1}$. 
Define $\alpha_{P}(S)$ to be the number of chains $\hat{0}<y_{1}<\cdots<y_{j-1}<\hat{1}$ in $P$ such that $\operatorname{rk}\left(y_{i}\right)=a_{i}$ for $1 \leq i \leq j-1$. The function $\alpha_{P}$ is called the flag $f$-vector of $P$.

Claim. $\alpha_{P}(S)$ is the number of maximal chains $\hat{0}=x_{0} \lessdot x_{1} \lessdot \cdots \lessdot x_{n}=\hat{1}$ such that

$$
\lambda\left(x_{i-1}, x_{i}\right)>\lambda\left(x_{i}, x_{i+1}\right) \Rightarrow i \in S, 1 \leq i \leq n .
$$

To prove the claim, let $\hat{0}=y_{0}<y_{1}<\cdots<y_{j-1}<y_{j}=\hat{1}$ with $\operatorname{rk}\left(y_{i}\right)=a_{i}$ for $1 \leq i \leq j-1$. By the definition of $E$-labeling, there exists a unique refinement

$$
\hat{0}=y_{0}=x_{0} \lessdot x_{1} \lessdot \cdots \lessdot x_{a_{1}}=y_{1} \lessdot x_{a_{1}+1} \lessdot \cdots \lessdot x_{a_{2}}=y_{2} \lessdot \cdots \lessdot x_{n}=y_{j}=\hat{1}
$$

satisfying

$$
\begin{gathered}
\lambda\left(x_{0}, x_{1}\right) \leq \lambda\left(x_{1}, x_{2}\right) \leq \cdots \leq \lambda\left(x_{a_{1}-1}, x_{a_{1}}\right) \\
\lambda\left(x_{a_{1}}, x_{a_{1}+1}\right) \leq \lambda\left(x_{a_{1}+1}, x_{a_{1}+2}\right) \leq \cdots \leq \lambda\left(x_{a_{2}-1}, x_{a_{2}}\right)
\end{gathered}
$$$$
\cdots
$$

Thus if $\lambda\left(x_{i-1}, x_{i}\right)>\lambda\left(x_{i}, x_{i+1}\right)$, then $i \in S$, so (27) is satisfied. Conversely, given a maximal chain $\hat{0}=x_{0} \lessdot x_{1} \lessdot \cdots \lessdot x_{n}=\hat{1}$ satisfying the above conditions on $\lambda$, let $y_{i}=x_{a_{i}}$. Therefore we have a bijection between the chains counted by $\alpha_{P}(S)$ and the maximal chains satisfying (27), so the claim follows.

Now for $S \subseteq[n-1]$ define

$$
\beta_{P}(S)=\sum_{T \subseteq S}(-1)^{\#(S-T)} \alpha_{P}(T) .
$$

The function $\beta_{P}$ is called the flag h-vector of $P$. A simple Inclusion-Exclusion argument gives

$$
\alpha_{P}(S)=\sum_{T \subseteq S} \beta_{P}(T)
$$

for all $S \subseteq[n-1]$. It follows from the claim and equation $(29)$ that $\beta_{P}(T)$ is equal to the number of maximal chains $\hat{0}=x_{0} \lessdot x_{1} \lessdot \cdots \lessdot x_{n}=\hat{1}$ such that $\lambda\left(x_{i}\right)>\lambda\left(x_{i+1}\right)$ if and only if $i \in T$. In particular, $\beta_{P}([n-1])$ is equal to the number of strictly decreasing maximal chains $\hat{0}=x_{0} \lessdot x_{1} \lessdot \cdots \lessdot x_{n}=\hat{1}$ of $P$, i.e.,

$$
\lambda\left(x_{0}, x_{1}\right)>\lambda\left(x_{1}, x_{2}\right)>\cdots>\lambda\left(x_{n-1}, x_{n}\right) .
$$

Now by (28) we have

$$
\begin{aligned}
\beta_{P}([n-1]) & =\sum_{T \subseteq[n-1]}(-1)^{n-1-\# T} \alpha_{P}(T) \\
& =\sum_{k \geq 1} \sum_{\hat{0}=y_{0}<y_{1}<\cdots<y_{k}=\hat{1}}(-1)^{n-k} \\
& =(-1)^{n} \sum_{k \geq 1}(-1)^{k} c_{k},
\end{aligned}
$$

where $c_{i}$ is the number of chains $\hat{0}=y_{0}<y_{1}<\cdots<y_{i}=\hat{1}$ in $P$. The proof now follows from Philip Hall's theorem (Lemma 4.4).

We come to the main result of this subsection, a combinatorial interpretation of the coefficients of the characteristic polynomial $\chi_{M}(t)$ for any matroid $M$. 


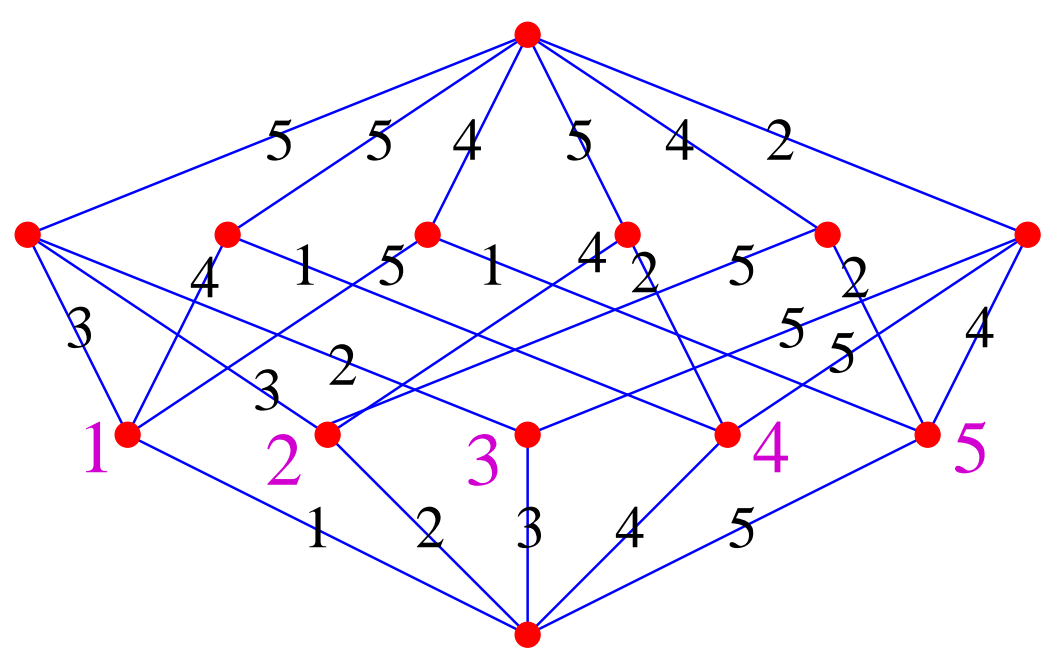

Figure 3. The edge labeling $\tilde{\lambda}$ of a geometric lattice $L(M)$

Theorem 4.12. Let $M$ be a matroid of rank $n$ with a linear ordering $x_{1}<x_{2}<$ $\cdots<x_{m}$ of its points (so the broken circuit complex $B C(M)$ is defined), and let $0 \leq i \leq n$. Then

$$
(-1)^{i}\left[t^{n-i}\right] \chi_{M}(t)=f_{i-1}(\mathrm{BC}(M)) .
$$

Proof. We may assume $M$ is simple since the "simplification" $\widehat{M}$ has the same lattice of flats and same broken circuit complex as $M$ (Exercise 1). The atoms $x_{i}$ of $L(M)$ can then be identified with the points of $M$. Define a labeling $\tilde{\lambda}: \mathcal{E}(L(M)) \rightarrow$ $\mathbb{P}$ as follows. Let $x \lessdot y$ in $L(M)$. Then set

$$
\tilde{\lambda}(x, y)=\max \left\{i: x \vee x_{i}=y\right\} .
$$

Note that $\tilde{\lambda}(x, y)$ is defined since $L(M)$ is atomic.

As an example, Figure 3 shows the lattice of flats of the matroid $M$ of Figure 1 with the edge labeling (30).

Claim 1. Define $\lambda: \mathcal{E}(L(M)) \rightarrow \mathbb{P}$ by

$$
\lambda(x, y)=m+1-\tilde{\lambda}(x, y) .
$$

Then $\lambda$ is an $E$-labeling.

To prove this claim, we need to show that for all $x<y$ in $L(M)$ there is a unique saturated chain $x=y_{0} \lessdot y_{1} \lessdot \cdots \lessdot y_{k}=y$ satisfying

$$
\tilde{\lambda}\left(y_{0}, y_{1}\right) \geq \tilde{\lambda}\left(y_{1}, y_{2}\right) \geq \cdots \geq \tilde{\lambda}\left(y_{k-1}, y_{k}\right) .
$$

The proof is by induction on $k$. There is nothing to prove for $k=1$. Let $k>1$ and assume the assertion for $k-1$. Let

$$
j=\max \left\{i: x_{i} \leq y, x_{i} \not \leq x\right\} .
$$

For any saturated chain $x=z_{0} \lessdot z_{1} \lessdot \cdots \lessdot z_{k}=y$, there is some $i$ for which $x_{j} \not \leq z_{i}$ and $x_{j} \leq z_{i+1}$. Hence $\tilde{\lambda}\left(z_{i}, z_{i+1}\right)=j$. Thus if $\tilde{\lambda}\left(z_{0}, z_{1}\right) \geq \cdots \geq \tilde{\lambda}\left(z_{k-1}, z_{k}\right)$, then $\tilde{\lambda}\left(z_{0}, z_{1}\right)=j$. Moreover, there is a unique $y_{1}$ satisfying $x=x_{0} \lessdot y_{1} \leq y$ and $\tilde{\lambda}\left(x_{0}, y_{1}\right)=j$, viz., $y_{1}=x_{0} \vee x_{j}$. (Note that $y_{1} \gg x_{0}$ by semimodularity.) 
By the induction hypothesis there exists a unique saturated chain $y_{1} \lessdot y_{2} \lessdot$ $\cdots \lessdot y_{k}=y$ satisfying $\tilde{\lambda}\left(y_{1}, y_{2}\right) \geq \cdots \geq \tilde{\lambda}\left(y_{k-1}, y_{k}\right)$. Since $\tilde{\lambda}\left(y_{0}, y_{1}\right)=j>\tilde{\lambda}\left(y_{1}, y_{2}\right)$, the proof of Claim 1 follows by induction.

Claim 2. The broken circuit complex $\mathrm{BC}(M)$ consists of all chain labels $\lambda(C)$, where $C$ is a saturated increasing chain (with respect to $\tilde{\lambda}$ ) from $\hat{0}$ to some $x \in$ $L(M)$. Moreover, all such $\lambda(C)$ are distinct.

To prove the distinctness of the labels $\lambda(C)$, suppose that $C$ is given by $\hat{0}=$ $y_{0} \lessdot y_{1} \lessdot \cdots \lessdot y_{k}$, with $\tilde{\lambda}(C)=\left(a_{1}, a_{2}, \ldots, a_{k}\right)$. Then $y_{i}=y_{i-1} \vee x_{a_{i}}$, so $C$ is the only chain with its label.

Now let $C$ and $\tilde{\lambda}(C)$ be as in the previous paragraph. We claim that the set $\left\{x_{a_{1}}, \ldots, x_{a_{k}}\right\}$ contains no broken circuit. (We don't even require that $C$ is increasing for this part of the proof.) Write $z_{i}=x_{a_{i}}$, and suppose to the contrary that $B=\left\{z_{i_{1}}, \ldots, z_{i_{j}}\right\}$ is a broken circuit, with $1 \leq i_{1}<\cdots<i_{j} \leq k$. Let $B \cup\left\{x_{r}\right\}$ be a circuit with $r>a_{i_{t}}$ for $1 \leq t \leq j$. Now for any circuit $\left\{u_{1}, \ldots, u_{h}\right\}$ and any $1 \leq i \leq h$ we have

$$
u_{1} \vee u_{2} \vee \cdots \vee u_{h}=u_{1} \vee \cdots \vee u_{i-1} \vee u_{i+1} \vee \cdots \vee u_{h}
$$

Thus

$$
z_{i_{1}} \vee z_{i_{2}} \vee \cdots \vee z_{i_{j-1}} \vee x_{r}=\bigvee_{z \in B} z=z_{i_{1}} \vee z_{i_{2}} \vee \cdots \vee z_{i_{j}}
$$

Then $y_{i_{j}-1} \vee x_{r}=y_{i_{j}}$, contradicting the maximality of the label $a_{i_{j}}$. Hence $\left\{x_{a_{1}}, \ldots, x_{a_{k}}\right\} \in \mathrm{BC}(M)$.

Conversely, suppose that $T:=\left\{x_{a_{1}}, \ldots, x_{a_{k}}\right\}$ contains no broken circuit, with $a_{1}<\cdots<a_{k}$. Let $y_{i}=x_{a_{1}} \vee \cdots \vee x_{a_{i}}$, and let $C$ be the chain $\hat{0}:=y_{0} \lessdot y_{1} \lessdot \cdots \lessdot y_{k}$. (Note that $C$ is saturated by semimodularity.) We claim that $\tilde{\lambda}(C)=\left(a_{1}, \ldots, a_{k}\right)$. If not, then $y_{i-1} \vee x_{j}=y_{i}$ for some $j>a_{i}$. Thus

$$
\operatorname{rk}(T)=\operatorname{rk}\left(T \cup\left\{x_{j}\right\}\right)=i .
$$

Since $T$ is independent, $T \cup\left\{x_{j}\right\}$ contains a circuit $Q$ satisfying $x_{j} \in Q$, so $T$ contains a broken circuit. This contradiction completes the proof of Claim 2.

To complete the proof of the theorem, note that we have shown that $f_{i-1}(\mathrm{BC}(M))$ is the number of chains $C: \hat{0}=y_{0} \lessdot y_{1} \lessdot \cdots \lessdot y_{i}$ such that $\tilde{\lambda}(C)$ is strictly increasing, or equivalently, $\lambda(C)$ is strictly decreasing. Since $\lambda$ is an $E$-labeling, the proof follows from Theorem 4.11.

Corollary 4.6. The broken circuit complex $\mathrm{BC}(M)$ is pure, i.e., every maximal face has the same dimension.

The proof is left as an exercise (Exercise 21).

Note (for readers with some knowledge of topology). (a) Let $M$ be a matroid on the linearly ordered set $u_{1}<u_{2}<\cdots<u_{m}$. Note that $F \in \mathrm{BC}(M)$ if and only if $F \cup\left\{u_{m}\right\} \in \mathrm{BC}(M)$. Define the reduced broken circuit complex $\mathrm{BC}_{r}(M)$ by

$$
\mathrm{BC}_{r}(M)=\left\{F \in \mathrm{BC}(M): u_{m} \notin F\right\} .
$$

Thus

$$
\mathrm{BC}(M)=\mathrm{BC}_{r}(M) * u_{m},
$$

the join of $\mathrm{BC}_{r}(M)$ and the vertex $u_{m}$. Equivalently, $\mathrm{BC}(M)$ is a cone over $\mathrm{BC}_{r}(M)$ with apex $u_{m}$. As a consequence, $\mathrm{BC}(M)$ is contractible and therefore has the homotopy type of a point. A more interesting problem is to determine the topological nature of $\mathrm{BC}_{r}(M)$. It can be shown that $\mathrm{BC}_{r}(M)$ has the homotopy type of a wedge 
of $\beta(M)$ spheres of dimension $\operatorname{rank}(M)-2$, where $(-1)^{\operatorname{rank}(M)-1} \beta(M)=\chi_{M}^{\prime}(1)$ (the derivative of $\chi_{M}(t)$ at $t=1$ ). See Exercise 22 for more information on $\beta(M)$.

As an example of the applicability of our results on matroids and geometric lattices to arrangements, we have the following purely combinatorial description of the number of regions of a real central arrangement.

Corollary 4.7. Let $\mathcal{A}$ be a central arrangement in $\mathbb{R}^{n}$, and let $M$ be the matroid defined by the normals to $H \in \mathcal{A}$, i.e., the independent sets of $M$ are the linearly independent normals. Then with respect to any linear ordering of the points of $M$, $r(\mathcal{A})$ is the total number of subsets of $M$ that don't contain a broken circuit.

Proof. Immediate from Theorems 2.5 and 4.12.

\subsection{Modular elements}

We next discuss a situation in which the characteristic polynomial $\chi_{M}(t)$ factors in a nice way.

Definition 4.12. An element $x$ of a geometric lattice $L$ is modular if for all $y \in L$ we have

$$
\operatorname{rk}(x)+\operatorname{rk}(y)=\operatorname{rk}(x \wedge y)+\operatorname{rk}(x \vee y) .
$$

Example 4.9. Let $L$ be a geometric lattice.

(a) $\hat{0}$ and $\hat{1}$ are clearly modular (in any finite lattice).

(b) We claim that atoms $a$ are modular.

Proof. Suppose that $a \leq y$. Then $a \wedge y=a$ and $a \vee y=y$, so equation (31) holds. (We don't need that $a$ is an atom for this case.) Now suppose $a \not \leq y$. By semimodularity, $\operatorname{rk}(a \vee y)=1+\operatorname{rk}(y)$, while $\operatorname{rk}(a)=1$ and $\operatorname{rk}(a \wedge y)=\operatorname{rk}(\hat{0})=0$, so again (31) holds.

(c) Suppose that $\operatorname{rk}(L)=3$. All elements of rank 0, 1, or 3 are modular by (a) and (b). Suppose that $\operatorname{rk}(x)=2$. Then $x$ is modular if and only if for all elements $y \neq x$ and $\operatorname{rk}(y)=2$, we have that $\operatorname{rk}(x \wedge y)=1$.

(d) Let $L=B_{n}$. If $x \in B_{n}$ then $\operatorname{rk}(x)=\# x$. Moreover, for any $x, y \in B_{n}$ we have $x \wedge y=x \cap y$ and $x \vee y=x \cup y$. Since for any finite sets $x$ and $y$ we have

$$
\# x+\# y=\#(x \cap y)+\#(x \cup y)
$$

it follows that every element of $B_{n}$ is modular. In other words, $B_{n}$ is a modular lattice.

(e) Let $q$ be a prime power and $\mathbb{F}_{q}$ the finite field with $q$ elements. Define $B_{n}(q)$ to be the lattice of subspaces, ordered by inclusion, of the vector space $\mathbb{F}_{q}^{n}$. Note that $B_{n}(q)$ is also isomorphic to the intersection lattice of the arrangement of all linear hyperplanes in the vector space $\mathbb{F}_{n}(q)$. Figure 4 shows the Hasse diagrams of $B_{2}(3)$ and $B_{3}(2)$.

Note that for $x, y \in B_{n}(q)$ we have $x \wedge y=x \cap y$ and $x \vee y=x+y$ (subspace sum). Clearly $B_{n}(q)$ is atomic: every vector space is the join (sum) of its one-dimensional subspaces. Moreover, $B_{n}(q)$ is graded of rank $n$, with rank function given by $\operatorname{rk}(x)=\operatorname{dim}(x)$. Since for any subspaces $x$ and $y$ we have

$$
\operatorname{dim}(x)+\operatorname{dim}(y)=\operatorname{dim}(x \cap y)+\operatorname{dim}(x+y),
$$



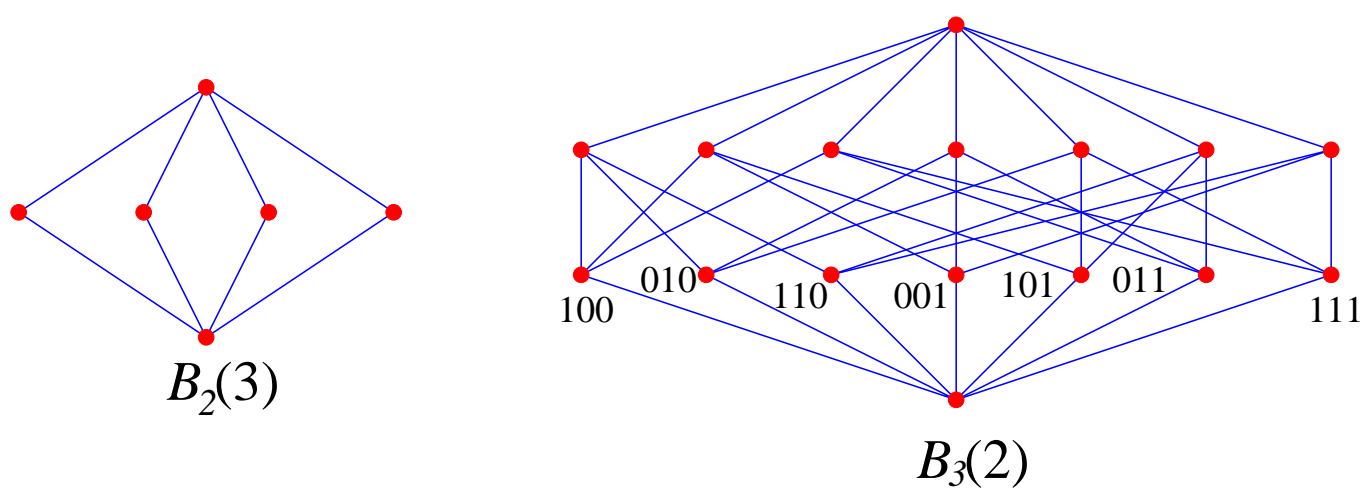

Figure 4. The lattices $B_{2}(3)$ and $B_{3}(2)$

it follows that $L$ is a modular geometric lattice. Thus every $x \in L$ is modular.

Note. A projective plane $R$ consists of a set (also denoted $R$ ) of points, and a collection of subsets of $R$, called lines, such that: (a) every two points lie on a unique line, (b) every two lines intersect in exactly one point, and (c) (non-degeneracy) there exist four points, no three of which are on a line. The incidence lattice $L(R)$ of $R$ is the set of all points and lines of $R$, ordered by $p<L$ if $p \in L$, with $\hat{0}$ and $\hat{1}$ adjoined. It is an immediate consequence of the axioms that when $R$ is finite, $L(R)$ is a modular geometric lattice of rank 3. It is an open (and probably intractable) problem to classify all finite projective planes. Now let $P$ and $Q$ be posets and define their direct product (or cartesian product) to be the set

$$
P \times Q=\{(x, y): x \in P, y \in Q\},
$$

ordered componentwise, i.e., $(x, y) \leq\left(x^{\prime}, y^{\prime}\right)$ if $x \leq x^{\prime}$ and $y \leq y^{\prime}$. It is easy to see that if $P$ and $Q$ are geometric (respectively, atomic, semimodular, modular) lattices, then so is $P \times Q$ (Exercise 7). It is a consequence of the "fundamental theorem of projective geometry" that every finite modular geometric lattice is a direct product of boolean algebras $B_{n}$, subspace lattices $B_{n}(q)$ for $n \geq 3$, lattices of rank 2 with at least five elements (which may be regarded as $B_{2}(q)$ for any $q \geq 2$ ) and incidence lattices of finite projective planes.

(f) The following result characterizes the modular elements of $\Pi_{n}$, which is the lattice of partitions of $[n]$ or the intersection lattice of the braid arrangement $\mathcal{B}_{n}$.

Proposition 4.9. A partition $\pi \in \Pi_{n}$ is a modular element of $\Pi_{n}$ if and only if $\pi$ has at most one nonsingleton block. Hence the number of modular elements of $\Pi_{n}$ is $2^{n}-n$.

Proof. If all blocks of $\pi$ are singletons, then $\pi=\hat{0}$, which is modular by (a). Assume that $\pi$ has the block $A$ with $r>1$ elements, and all other blocks are singletons. Hence the number $|\pi|$ of blocks of $\pi$ is given by 
$n-r+1$. For any $\sigma \in \Pi_{n}$, we have $\operatorname{rk}(\sigma)=n-|\sigma|$. Let $k=|\sigma|$ and

$$
j=\#\{B \in \sigma: A \cap B \neq \emptyset\} .
$$

Then $|\pi \wedge \sigma|=j+(n-r)$ and $|\pi \vee \sigma|=k-j+1$. Hence $\operatorname{rk}(\pi)=r-1$, $\operatorname{rk}(\sigma)=n-k, \operatorname{rk}(\pi \wedge \sigma)=r-j$, and $\operatorname{rk}(\pi \vee \sigma)=n-k+j-1$, so $\pi$ is modular.

Conversely, let $\pi=\left\{B_{1}, B_{2}, \ldots, B_{k}\right\}$ with $\# B_{1}>1$ and $\# B_{2}>1$. Let $a \in B_{1}$ and $b \in B_{2}$, and set

$$
\sigma=\left\{\left(B_{1} \cup b\right)-a,\left(B_{2} \cup a\right)-b, B_{3}, \ldots, B_{k}\right\} .
$$

Then

$$
\begin{array}{cc}
|\pi|=|\sigma|=k & \\
\pi \wedge \sigma=\left\{a, b, B_{1}-a, B_{2}-b, \ldots, B_{3}, \ldots, B_{k}\right\} & \Rightarrow|\pi \wedge \sigma|=k+2 \\
\pi \vee \sigma=\left\{B_{1} \cup B_{2}, B_{3}, \ldots, B_{l}\right\} & \Rightarrow|\pi \vee \sigma|=k-1 .
\end{array}
$$

Hence $\operatorname{rk}(\pi)+\operatorname{rk}(\sigma) \neq \operatorname{rk}(\pi \wedge \sigma)+\operatorname{rk}(\pi \vee \sigma)$, so $\pi$ is not modular.

In a finite lattice $L$, a complement of $x \in L$ is an element $y \in L$ such that $x \wedge y=\hat{0}$ and $x \vee y=\hat{1}$. For instance, in the boolean algebra $B_{n}$ every element has a unique complement. (See Exercise 3 for the converse.) The following proposition collects some useful properties of modular elements. The proof is left as an exercise (Exercises 4-5).

Proposition 4.10. Let $L$ be a geometric lattice of rank $n$.

(a) Let $x \in L$. The following four conditions are equivalent.

(i) $x$ is a modular element of $L$.

(ii) If $x \wedge y=\hat{0}$, then $\operatorname{rk}(x)+\operatorname{rk}(y)=\operatorname{rk}(x \vee y)$.

(iii) If $x$ and $y$ are complements, then $\operatorname{rk}(x)+\operatorname{rk}(y)=n$.

(iv) All complements of $x$ are incomparable.

(b) (transitivity of modularity) If $x$ is a modular element of $L$ and $y$ is modular in the interval $[\hat{0}, x]$, then $y$ is a modular element of $L$.

(c) If $x$ and $y$ are modular elements of $L$, then $x \wedge y$ is also modular.

The next result, known as the modular element factorization theorem [28], is our primary reason for defining modular elements - such an element induces a factorization of the characteristic polynomial.

Theorem 4.13. Let $z$ be a modular element of the geometric lattice $L$ of rank $n$. Write $\chi_{z}(t)=\chi_{[\hat{0}, z]}(t)$. Then

$$
\chi_{L}(t)=\chi_{z}(t)\left[\sum_{y: y \wedge z=0} \mu_{L}(y) t^{n-\operatorname{rk}(y)-\operatorname{rk}(z)}\right] .
$$

Example 4.10. Before proceeding to the proof of Theorem 4.13, let us consider an example. The illustration below is the affine diagram of a matroid $M$ of rank 3 , together with its lattice of flats. The two lines (flats of rank 2) labelled $x$ and $y$ are modular by Example 4.9(c). 

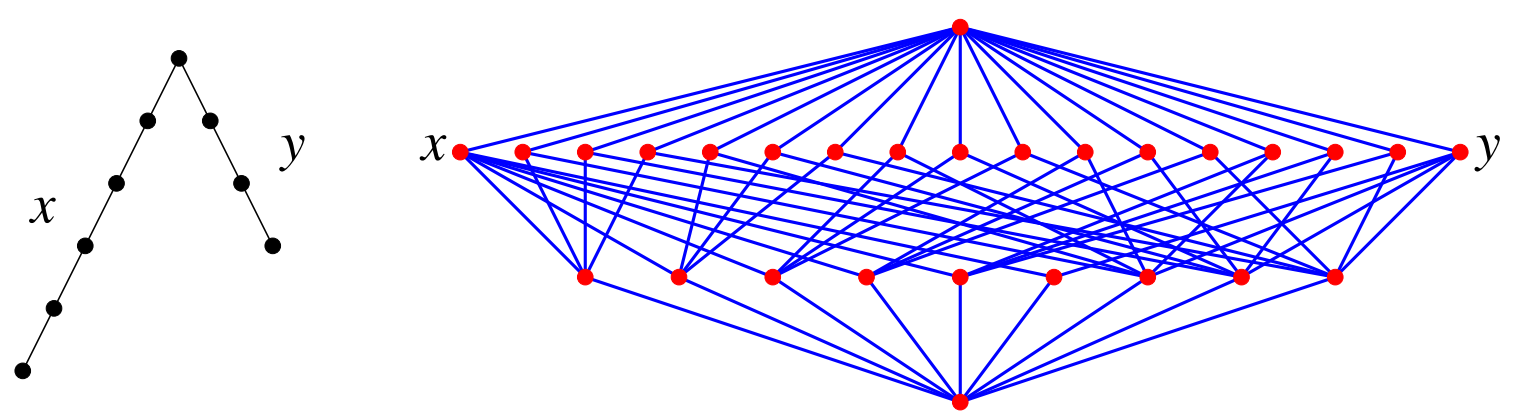

Hence by equation $(32) \chi_{M}(t)$ is divisible by $\chi_{x}(t)$. Moreover, any atom $a$ of the interval $[\hat{0}, x]$ is modular, so $\chi_{x}(t)$ is divisible by $\chi_{a}(t)=t-1$. From this it is immediate (e.g., because the characteristic polynomial $\chi_{G}(t)$ of any geometric lattice $G$ of rank $n$ begins $x^{n}-a x^{n-1}+\cdots$, where $a$ is the number of atoms of $G$ ) that $\chi_{x}(t)=(t-1)(t-5)$ and $\chi_{M}(t)=(t-1)(t-3)(t-5)$. On the other hand, since $y$ is modular, $\chi_{M}(t)$ is divisible by $\chi_{y}(t)$, and we get as before $\chi_{y}(t)=(t-1)(t-3)$ and $\chi_{M}(t)=(t-1)(t-3)(t-5)$. Geometric lattices whose characteristic polynomial factors into linear factors in a similar way due to a maximal chain of modular elements are discussed further beginning with Definition 4.13.

Our proof of Theorem 4.13 will depend on the following lemma of Greene [19]. We give a somewhat simpler proof than Greene.

Lemma 4.5. Let $L$ be a finite lattice with Möbius function $\mu$, and let $z \in L$. The following identity is valid in the Möbius algebra $A(L)$ of $L$ :

$$
\sigma_{\hat{0}}:=\sum_{x \in L} \mu(x) x=\left(\sum_{v \leq z} \mu(v) v\right)\left(\sum_{y \wedge z=\hat{0}} \mu(y) y\right) .
$$


Proof. Let $\sigma_{s}$ for $s \in L$ be given by (8). The right-hand side of equation (33) is then given by

$$
\begin{aligned}
& \sum_{\substack{v \leq z \\
y \wedge z=0}} \mu(v) \mu(y)(v \vee y)=\sum_{\substack{v \leq z \\
y \wedge z=0}} \mu(v) \mu(y) \sum_{s \geq v \vee y} \sigma_{s} \\
& =\sum_{s} \sigma_{s} \sum_{\substack{v \leq s, v \leq z \\
y \leq s, y \wedge z=0 \hat{0}}} \mu(v) \mu(y) \\
& =\sum_{s} \sigma_{s}(\underbrace{\sum_{v \leq s \wedge z} \mu(v)}_{\delta_{\hat{0}, s \wedge z}})\left(\sum_{\substack{y \leq s \\
y \wedge z=0}} \mu(y)\right) \\
& =\sum_{s \wedge z=\hat{0}} \sigma_{s}(\underbrace{\sum_{\substack{y \leq s \\
y \wedge z=0}} \mu(y)}_{\delta_{\hat{0}, s}}) \\
& =\sigma_{\hat{0}} \text {. }
\end{aligned}
$$

Proof of Theorem 4.13. We are assuming that $z$ is a modular element of the geometric lattice $L$.

Claim 1. Let $v \leq z$ and $y \wedge z=\hat{0}$ (so $v \wedge y=\hat{0})$. Then $z \wedge(v \vee y)=v$ (as illustrated below).

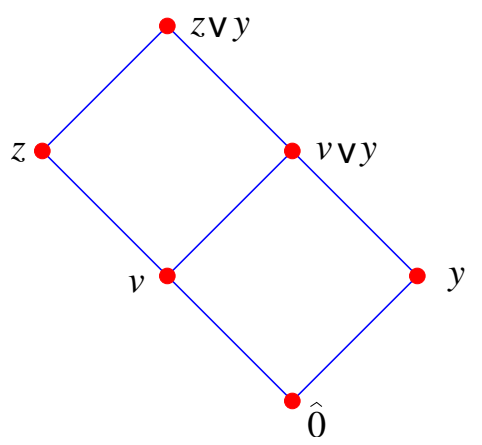


Proof of Claim 1. Clearly $z \wedge(v \vee y) \geq v$, so it suffices to show that $\operatorname{rk}(z \wedge(v \vee$ $y)) \leq \operatorname{rk}(v)$. Since $z$ is modular we have

$$
\begin{aligned}
\operatorname{rk}(z \wedge(v \vee y)) & =\operatorname{rk}(z)+\operatorname{rk}(v \vee y)-\operatorname{rk}(z \vee y) \\
& =\operatorname{rk}(z)+\operatorname{rk}(v \vee y)-(\operatorname{rk}(z)+\operatorname{rk}(y)-\underbrace{\operatorname{rk}(z \wedge y)}_{0}) \\
& =\operatorname{rk}(v \vee y)-\operatorname{rk}(y) \\
& \leq(\operatorname{rk}(v)+\operatorname{rk}(y)-\underbrace{\operatorname{rk}(v \wedge y)}_{0})-\operatorname{rk}(y) \text { by semimodularity } \\
& =\operatorname{rk}(v),
\end{aligned}
$$

proving Claim 1.

Claim 2. With $v$ and $y$ as above, we have $\operatorname{rk}(v \vee y)=\operatorname{rk}(v)+\operatorname{rk}(y)$.

Proof of Claim 2. By the modularity of $z$ we have

$$
\operatorname{rk}(z \wedge(v \vee y))+\operatorname{rk}(z \vee(v \vee y))=\operatorname{rk}(z)+\operatorname{rk}(v \vee y)
$$

By Claim 1 we have $\operatorname{rk}(z \wedge(v \vee y))=\operatorname{rk}(v)$. Moreover, again by the modularity of $z$ we have

$$
\operatorname{rk}(z \vee(v \vee y))=\operatorname{rk}(z \vee y)=\operatorname{rk}(z)+\operatorname{rk}(y)-\operatorname{rk}(z \wedge y)=\operatorname{rk}(z)+\operatorname{rk}(y)
$$

It follows that $\operatorname{rk}(v)+\operatorname{rk}(y)=\operatorname{rk}(v \vee y)$, as claimed.

Now substitute $\mu(v) v \rightarrow \mu(v) t^{\operatorname{rk}(z)-\operatorname{rk}(v)}$ and $\mu(y) y \rightarrow \mu(y) t^{n-\operatorname{rk}(y)-\operatorname{rk}(z)}$ in the right-hand side of equation (33). Then by Claim 2 we have

$$
v y \rightarrow t^{n-\operatorname{rk}(v)-\mathrm{rk}(y)}=t^{n-\mathrm{rk}(v \vee y)} .
$$

Now $v \vee y$ is just $v y$ in the Möbius algebra $A(L)$. Hence if we further substitute $\mu(x) x \rightarrow \mu(x) t^{n-\operatorname{rk}(x)}$ in the left-hand side of (33), then the product will be preserved. We thus obtain

$$
\underbrace{\sum_{x \in L} \mu(x) t^{n-\operatorname{rk}(x)}}_{\chi_{L}(t)}=(\underbrace{\sum_{v \leq z} \mu(v) t^{\mathrm{rk}(z)-\mathrm{rk}(v)}}_{\chi_{z}(t)})\left(\sum_{y \wedge z=\hat{0}} \mu(y) t^{n-\mathrm{rk}(y)-\mathrm{rk}(z)}\right),
$$

as desired.

Corollary 4.8. Let $L$ be a geometric lattice of rank $n$ and a an atom of $L$. Then

$$
\chi_{L}(t)=(t-1) \sum_{y \wedge a=\hat{0}} \mu(y) t^{n-1-\operatorname{rk}(y)} .
$$

Proof. The atom $a$ is modular (Example $4.9(\mathrm{~b}))$, and $\chi_{a}(t)=t-1$.

Corollary 4.8 provides a nice context for understanding the operation of coning defined in Chapter 1, in particular, Exercise 2.1. Recall that if $\mathcal{A}$ is an affine arrangement in $K^{n}$ given by the equations

$$
L_{1}(x)=a_{1}, \ldots, L_{m}(x)=a_{m},
$$

then the cone $x \mathcal{A}$ is the arrangement in $K^{n} \times K$ (where $y$ denotes the last coordinate) with equations

$$
L_{1}(x)=a_{1} y, \ldots, L_{m}(x)=a_{m} y, y=0 .
$$


Let $H_{0}$ denote the hyperplane $y=0$. It is easy to see by elementary linear algebra that

$$
L(\mathcal{A}) \cong L(c \mathcal{A})-\left\{x \in L(\mathcal{A}): x \geq H_{0}\right\}=L(\mathcal{A})-L\left(\mathcal{A}^{H_{0}}\right) .
$$

Now $H_{0}$ is a modular element of $L(\mathcal{A})$ (since it's an atom), so Corollary 4.8 yields

$$
\begin{aligned}
\chi_{c \mathcal{A}}(t)=(t-1) \sum_{y \nsupseteq H_{0}} \mu(y) t^{(n+1)-1-\operatorname{rk}(y)} & \\
& =(t-1) \chi_{\mathcal{A}}(t) .
\end{aligned}
$$

There is a left inverse to the operation of coning. Let $\mathcal{A}$ be a nonempty linear arrangement in $K^{n+1}$. Let $H_{0} \in \mathcal{A}$. Choose coordinates $\left(x_{0}, x_{1}, \ldots, x_{n}\right)$ in $K^{n+1}$ so that $H_{0}=\operatorname{ker}\left(x_{0}\right)$. Let $\mathcal{A}$ be defined by the equations

$$
x_{0}=0, L_{1}\left(x_{0}, \ldots, x_{n}\right)=0, \ldots, L_{m}\left(x_{0}, \ldots, x_{n}\right)=0 .
$$

Define the deconing $c^{-1} \mathcal{A}$ (with respect to $H_{0}$ ) in $K^{n}$ by the equations

$$
L_{1}\left(1, x_{1}, \ldots, x_{n}\right)=0, \ldots L_{m}\left(1, x_{1}, \ldots, x_{n}\right)=0 .
$$

Clearly $c\left(c^{-1} \mathcal{A}\right)=\mathcal{A}$ and $L\left(c^{-1} \mathcal{A}\right) \cong L(\mathcal{A})-\left\{x \in L(\mathcal{A}): x \geq H_{0}\right\}$.

\subsection{Supersolvable lattices}

For some geometric lattices $L$, there are "enough" modular elements to give a factorization of $\chi_{L}(t)$ into linear factors.

Definition 4.13. A geometric lattice $L$ is supersolvable if there exists a modular maximal chain, i.e., a maximal chain $\hat{0}=x_{0} \lessdot x_{1} \lessdot \cdots \lessdot x_{n}=\hat{1}$ such that each $x_{i}$ is modular. A central arrangement $\mathcal{A}$ is supersolvable if its intersection lattice $L_{\mathcal{A}}$ is supersolvable.

Note. Let $\hat{0}=x_{0} \lessdot x_{1} \lessdot \cdots \lessdot x_{n}=\hat{1}$ be a modular maximal chain of the geometric lattice $L$. Clearly then each $x_{i-1}$ is a modular element of the interval $\left[\hat{0}, x_{i}\right]$. The converse follows from Proposition 4.10(b): if $\hat{0}=x_{0} \lessdot x_{1} \lessdot \cdots \lessdot x_{n}=\hat{1}$ is a maximal chain for which each $x_{i-1}$ is modular in [0, $\left.x_{i}\right]$, then each $x_{i}$ is modular in $L$.

Note. The term "supersolvable" comes from group theory. A finite group $\Gamma$ is supersolvable if and only if its subgroup lattice contains a maximal chain all of whose elements are normal subgroups of $\Gamma$. Normal subgroups are "nice" analogues of modular elements; see [29, Example 2.5] for further details.

Corollary 4.9. Let $L$ be a supersolvable geometric lattice of rank $n$, with modular maximal chain $\hat{0}=x_{0} \lessdot x_{1} \lessdot \cdots \lessdot x_{n}=\hat{1}$. Let $T$ denote the set of atoms of $L$, and set

$$
e_{i}=\#\left\{a \in T: a \leq x_{i}, a \not \leq x_{i-1}\right\} .
$$

Then $\chi_{L}(t)=\left(t-e_{1}\right)\left(t-e_{2}\right) \cdots\left(t-e_{n}\right)$.

Proof. Since $x_{n-1}$ is modular, we have

$$
y \wedge x_{n-1}=\hat{0} \Leftrightarrow y \in T \text { and } y \not \leq x_{n-1}, \text { or } y=\hat{0} .
$$


By Theorem 4.13 we therefore have

$$
\chi_{L}(t)=\chi_{x_{n-1}}(t)\left[\sum_{\substack{a \in T \\ a \nsubseteq x_{n-1}}} \mu(a) t^{n-\mathrm{rk}(a)-\mathrm{rk}\left(x_{n-1}\right)}+\mu(\hat{0}) t^{n-\mathrm{rk}(\hat{0})-\mathrm{rk}\left(x_{n-1}\right)}\right] .
$$

Since $\mu(a)=-1, \mu(\hat{0})=1, \operatorname{rk}(a)=1, \operatorname{rk}(\hat{0})=0$, and $\operatorname{rk}\left(x_{n-1}\right)=n-1$, the expression in brackets is just $t-e_{n}$. Now continue this with $L$ replaced by $\left[\hat{0}, x_{n-1}\right]$ (or use induction on $n$ ).

Note. The positive integers $e_{1}, \ldots, e_{n}$ of Corollary 4.9 are called the exponents of $L$.

Example 4.11. (a) Let $L=B_{n}$, the boolean algebra of rank $n$. By Example $4.9(\mathrm{~d})$ every element of $B_{n}$ is modular. Hence $B_{n}$ is supersolvable. Clearly each $e_{i}=1$, so $\chi_{B_{n}}(t)=(t-1)^{n}$.

(b) Let $L=B_{n}(q)$, the lattice of subspaces of $\mathbb{F}_{n}^{q}$. By Example 4.9(e) every element of $B_{n}(q)$ is modular, so $B_{n}(q)$ is supersolvable. If $\left[\begin{array}{l}k \\ j\end{array}\right]$ denotes the number of $j$-dimensional subspaces of a $k$-dimensional vector space over $\mathbb{F}_{q}$, then

$$
\begin{aligned}
e_{i} & =\left[\begin{array}{l}
i \\
1
\end{array}\right]-\left[\begin{array}{c}
i-1 \\
1
\end{array}\right] \\
& =\frac{q^{i}-1}{q-1}-\frac{q^{i-1}-1}{q-1} \\
& =q^{i-1} .
\end{aligned}
$$

Hence

$$
\chi_{B_{n}(q)}(t)=(t-1)(t-q)\left(t-q^{2}\right) \cdots\left(t-q^{n-1}\right) .
$$

In particular, setting $t=0$ gives

$$
\mu_{B_{n}(q)}(\hat{1})=(-1)^{n} q^{\left(\begin{array}{c}
n \\
2
\end{array}\right)} .
$$

Note. The expression $\left[\begin{array}{l}k \\ j\end{array}\right]$ is called a $q$-binomial coefficient. It is a polynomial in $q$ with many interesting properties. For the most basic properties, see e.g. [31, pp. 27-30].

(c) Let $L=\Pi_{n}$, the lattice of partitions of the set [n] (a geometric lattice of rank $n-1)$. By Proposition 4.9, a maximal chain of $\Pi_{n}$ is modular if and only if it has the form $\hat{0}=\pi_{0} \lessdot \pi_{1} \lessdot \cdots \lessdot \pi_{n-1}=\hat{1}$, where $\pi_{i}$ for $i>0$ has exactly one nonsingleton block $B_{i}$ (necessarily with $i+1$ elements), with $B_{1} \subset B_{2} \cdots \subset B_{n-1}=[n]$. In particular, $\Pi_{n}$ is supersolvable and has exactly $n ! / 2$ modular chains for $n>1$. The atoms covered by $\pi_{i}$ are the partitions with one nonsingleton block $\{j, k\} \subseteq B_{i}$. Hence $\pi_{i}$ lies above exactly $\left(\begin{array}{c}i+1 \\ 2\end{array}\right)$ atoms, so

$$
e_{i}=\left(\begin{array}{c}
i+1 \\
2
\end{array}\right)-\left(\begin{array}{l}
i \\
2
\end{array}\right)=i
$$

It follows that $\chi_{\Pi_{n}}(t)=(t-1)(t-2) \cdots(t-n+1)$ and $\mu_{\Pi_{n}}(\hat{1})=$ $(-1)^{n-1}(n-1)$ !. Compare Corollary 2.2. The polynomials $\chi_{\mathcal{B}_{n}}(t)$ and $\chi_{\Pi_{n}}(t)$ differ by a factor of $t$ because $\mathcal{B}_{n}(t)$ is an arrangement in $K^{n}$ of 
rank $n-1$. In general, if $\mathcal{A}$ is an arrangement and ess $(\mathcal{A})$ its essentialization, then

$$
t^{\mathrm{rk}(\operatorname{ess}(\mathcal{A}))} \chi_{\mathcal{A}}(t)=t^{\mathrm{rk}(\mathcal{A})} \chi_{\mathrm{ess}(\mathcal{A})}(t)
$$

(See Lecture 1, Exercise 2.)

Note. It is natural to ask whether there is a more general class of geometric lattices $L$ than the supersolvable ones for which $\chi_{L}(t)$ factors into linear factors (over $\mathbb{Z}$ ). There is a profound such generalization due to Terao [35] when $L$ is an intersection poset of a linear arrangement $\mathcal{A}$ in $K^{n}$. Write $K[x]=K\left[x_{1}, \ldots, x_{n}\right]$ and define

$$
\mathcal{T}(\mathcal{A})=\left\{\left(p_{1}, \ldots, p_{n}\right) \in K[x]^{n}: p_{i}(H) \subseteq H \text { for all } H \in \mathcal{A}\right\} .
$$

Here we are regarding $\left(p_{1}, \ldots, p_{n}\right): K^{n} \rightarrow K^{n}$, viz., if $\left(a_{1}, \ldots, a_{n}\right) \in K^{n}$, then

$$
\left(p_{1}, \ldots, p_{n}\right)\left(a_{1}, \ldots, a_{n}\right)=\left(p_{1}\left(a_{1}, \ldots, a_{n}\right), \ldots, p_{n}\left(a_{1}, \ldots, a_{n}\right)\right) .
$$

The $K[x]$-module structure $K[x] \times \mathcal{T}(\mathcal{A}) \rightarrow \mathcal{T}(\mathcal{A})$ is given explicitly by

$$
q \cdot\left(p_{1}, \ldots, p_{n}\right)=\left(q p_{1}, \ldots, q p_{n}\right) .
$$

Note, for instance, that we always have $\left(x_{1}, \ldots, x_{n}\right) \in \mathcal{T}(\mathcal{A})$. Since $\mathcal{A}$ is a linear arrangement, $\mathcal{T}(\mathcal{A})$ is indeed a $K[x]$-module. (We have given the most intuitive definition of the module $\mathcal{T}(\mathcal{A})$, though it isn't the most useful definition for proofs.) It is easy to see that $\mathcal{T}(\mathcal{A})$ has rank $n$ as a $K[x]$-module, i.e., $\mathcal{T}(\mathcal{A})$ contains $n$, but not $n+1$, elements that are linearly independent over $K[x]$. We say that $\mathcal{A}$ is a free arrangement if $\mathcal{T}(\mathcal{A})$ is a free $K[x]$-module, i.e., there exist $Q_{1}, \ldots, Q_{n} \in$ $\mathcal{T}(\mathcal{A})$ such that every element $Q \in \mathcal{T}(\mathcal{A})$ can be uniquely written in the form $Q=q_{1} Q_{1}+\cdots+q_{n} Q_{n}$, where $q_{i} \in K[x]$. It is easy to see that if $\mathcal{T}(\mathcal{A})$ is free, then the basis $\left\{Q_{1}, \ldots, Q_{n}\right\}$ can be chosen to be homogeneous, i.e., all coordinates of each $Q_{i}$ are homogeneous polynomials of the same degree $d_{i}$. We then write $d_{i}=\operatorname{deg} Q_{i}$. It can be shown that supersolvable arrangements are free, but there are also nonsupersolvable free arrangements. The property of freeness seems quite subtle; indeed, it is unknown whether freeness is a matroidal property, i.e., depends only on the intersection lattice $L_{\mathcal{A}}$ (regarding the ground field $K$ as fixed). The remarkable "factorization theorem" of Terao is the following.

Theorem 4.14. Suppose that $\mathcal{T}(\mathcal{A})$ is free with homogeneous basis $Q_{1}, \ldots, Q_{n}$. If $\operatorname{deg} Q_{i}=d_{i}$ then

$$
\chi_{\mathcal{A}}(t)=\left(t-d_{1}\right)\left(t-d_{2}\right) \cdots\left(t-d_{n}\right) .
$$
Ch. 4].

We will not prove Theorem 4.14 here. A good reference for this subject is $[\mathbf{2 4}$,

Returning to supersolvability, we can try to characterize the supersolvable property for various classes of geometric lattices. Let us consider the case of the bond lattice $L_{G}$ of the graph $G$. A graph $H$ with at least one edge is doubly connected if it is connected and remains connected upon the removal of any vertex (and all incident edges). A maximal doubly connected subgraph of a graph $G$ is called a block of $G$. For instance, if $G$ is a forest then its blocks are its edges. Two different blocks of $G$ intersect in at most one vertex. Figure 5 shows a graph with eight blocks, five of which consist of a single edge. The following proposition is straightforward to prove (Exercise 16). 


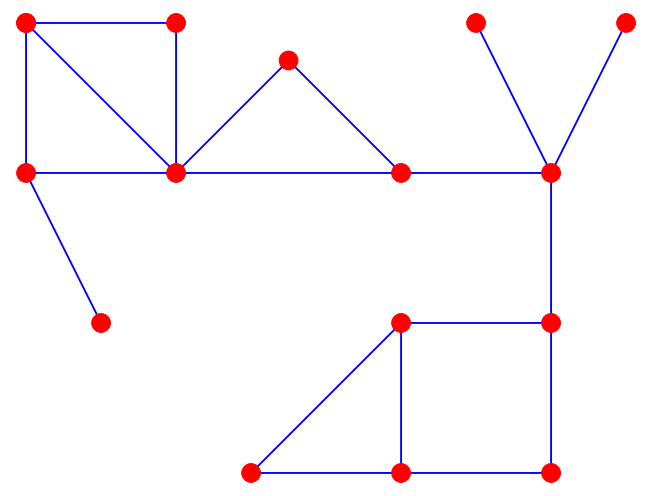

Figure 5. A graph with eight blocks

Proposition 4.11. Let $G$ be a graph with blocks $G_{1}, \ldots, G_{k}$. Then

$$
L_{G} \cong L_{G_{1}} \times \cdots \times L_{G_{k}} .
$$

It is also easy to see that if $L_{1}$ and $L_{2}$ are geometric lattices, then $L_{1}$ and $L_{2}$ are supersolvable if and only if $L_{1} \times L_{2}$ is supersolvable (Exercise 18). Hence in characterizing supersolvable graphs $G$ (i.e., graphs whose bond lattice $L_{G}$ is supersolvable) we may assume that $G$ is doubly connected. Note that for any connected (and hence a fortiori doubly connected) graph $G$, any coatom $\pi$ of $L_{G}$ has exactly two blocks.

Proposition 4.12. Let $G$ be a doubly connected graph, and let $\pi=\{A, B\}$ be a coatom of the bond lattice $L_{G}$, where $\# A \leq \# B$. Then $\pi$ is a modular element of $L_{G}$ if and only if $\# A=1$, say $A=\{v\}$, and the neighborhood $N(v)$ (the set of vertices adjacent to $v$ ) forms a clique (i.e., any two distinct vertices of $N(v)$ are adjacent).

Proof. The proof parallels that of Proposition 4.9, which is a special case. Suppose that $\# A>1$. Since $G$ is doubly connected, there exist $u, v \in A$ and $u^{\prime}, v^{\prime} \in B$ such that $u \neq v, u^{\prime} \neq v^{\prime}, u u^{\prime} \in E(G)$, and $v v^{\prime} \in E(G)$. Set $\sigma=\left\{\left(A \cup u^{\prime}\right)-v,(B \cup v)-u^{\prime}\right\}$. If $G$ has $n$ vertices then $\operatorname{rk}(\pi)=\operatorname{rk}(\sigma)=n-2, \operatorname{rk}(\pi \vee \sigma)=n-1$, and $\operatorname{rk}(\pi \wedge \sigma)=n-4$. Hence $\pi$ is not modular.

Assume then that $A=\{v\}$. Suppose that $a v, b v \in E(G)$ but $a b \notin E(G)$. We need to show that $\pi$ is not modular. Let $\sigma=\{A-\{a, b\},\{a, b, v\}\}$. Then

$$
\begin{gathered}
\sigma \vee \pi=\hat{1}, \quad \sigma \wedge \pi=\{A-\{a, b\}, a, b, v\} \\
\operatorname{rk}(\sigma)=\operatorname{rk}(\pi)=n-2, \quad \operatorname{rk}(\sigma \vee \pi)=n-1, \quad \operatorname{rk}(\sigma \wedge \pi)=n-4 .
\end{gathered}
$$

Hence $\pi$ is not modular.

Conversely, let $\pi=\{A, v\}$. Assume that if $a v, b v \in E(G)$ then $a b \in E(G)$. It is then straightforward to show (Exercise 8 ) that $\pi$ is modular, completing the proof.

As an immediate consequence of Propositions 4.10(b) and 4.12 we obtain a characterization of supersolvable graphs.

Corollary 4.10. A graph $G$ is supersolvable if and only if there exists an ordering $v_{1}, v_{2}, \ldots, v_{n}$ of its vertices such that if $i<k, j<k, v_{i} v_{k} \in E(G)$ and $v_{j} v_{k} \in E(G)$, 
then $v_{i} v_{j} \in E(G)$. Equivalently, in the restriction of $G$ to the vertices $v_{1}, v_{2}, \ldots, v_{i}$, the neighborhood of $v_{i}$ is a clique.

Note. Supersolvable graphs $G$ had appeared earlier in the literature under the names chordal, rigid circuit, or triangulated graphs. One of their many characterizations is that any circuit of length at least four contains a chord. Equivalently, no induced subgraph of $G$ is a $k$-cycle for $k \geq 4$. 


\section{Exercises}

(1) $[2-]$ Let $M$ be a matroid on a linearly ordered set. Show that $\mathrm{BC}(M)=\mathrm{BC}(\widehat{M})$, where $\widehat{M}$ is defined by equation (23).

(2) $[2+]$ Let $M$ be a matroid of rank at least one. Show that the coefficients of the polynomial $\chi_{M}(t) /(t-1)$ alternate in sign.

(3) (a) $[2+]$ Let $L$ be finite lattice for which every element has a unique complement. Show that $L$ is isomorphic to a boolean algebra $B_{n}$.

(b) [3] A lattice $L$ is distributive if

$$
\begin{aligned}
& x \vee(y \wedge z)=(x \vee y) \wedge(x \vee z) \\
& x \wedge(y \vee z)=(x \wedge y) \vee(x \wedge z)
\end{aligned}
$$

for all $x, y, z \in L$. Let $L$ be an infinite lattice with $\hat{0}$ and $\hat{1}$. If every element of $L$ has a unique complement, then is $L$ a distributive lattice?

(4) [3-] Let $x$ be an element of a geometric lattice $L$. Show that the following four conditions are equivalent.

(i) $x$ is a modular element of $L$.

(ii) If $x \wedge y=\hat{0}$, then

$$
\operatorname{rk}(x)+\operatorname{rk}(y)=\operatorname{rk}(x \vee y) .
$$

(iii) If $x$ and $y$ are complements, then $\operatorname{rk}(x)+\operatorname{rk}(y)=n$.

(iv) All complements of $x$ are incomparable.

(5) $[2+]$ Let $x, y$ be modular elements of a geometric lattice $L$. Show that $x \wedge y$ is also modular.

(6) [2] Let $L$ be a geometric lattice. Prove or disprove: if $x$ is modular in $L$ and $y$ is modular in the interval $[x, \hat{1}]$, then $y$ is modular in $L$.

(7) [2-] Let $L$ and $L^{\prime}$ be finite lattices. Show that if both $L$ and $L^{\prime}$ are geometric (respectively, atomic, semimodular, modular) lattices, then so is $L \times L^{\prime}$.

(8) [2] Let $G$ be a (loopless) connected graph and $v \in V(G)$. Let $A=V(G)-v$ and $\pi=\{A, v\} \in L_{G}$. Suppose that whenever $a v, b v \in E(G)$ we have $a b \in E(G)$. Show that $\pi$ is a modular element of $L_{G}$.

(9) $[2+]$ Generalize the previous exercise as follows. Let $G$ be a doubly-connected graph with lattice of contractions $L_{G}$. Let $\pi \in L_{G}$. Show that the following two conditions are equivalent.

(a) $\pi$ is a modular element of $L_{G}$.

(b) $\pi$ satisfies the following two properties:

(i) At most one block $B$ of $\pi$ contains more than one vertex of $G$.

(ii) Let $H$ be the subgraph induced by the block $B$ of (i). Let $K$ be any connected component of the subgraph induced by $G-B$, and let $H_{1}$ be the graph induced by the set of vertices in $H$ that are connected to some vertex in $K$. Then $H_{1}$ is a clique (complete subgraph) of $G$.

(10) $[2+]$ Let $L$ be a geometric lattice of rank $n$, and fix $x \in L$. Show that

$$
\chi_{L}(t)=\sum_{\substack{y \in L \\ x \wedge y=\hat{0}}} \mu(y) \chi_{L_{y}}(t) t^{n-\mathrm{rk}(x \vee y)},
$$

where $L_{y}$ is the image of the interval $[\hat{0}, x]$ under the map $z \mapsto z \vee y$.

(11) $[2+]$ Let $\mathcal{J}(M)$ be the set of independent sets of a matroid $M$. Find another matroid $N$ and a labeling of its points for which $\mathcal{J}(M)=\mathrm{BC}_{r}(N)$, the reduced broken circuit complex of $N$. 
(12) (a) $[2+]$ If $\Delta$ and $\Gamma$ are simplicial complexes on disjoint sets $A$ and $B$, respectively, then define the join $\Delta * \Gamma$ to be the simplicial complex on the set $A \cup B$ with faces $F \cup G$, where $F \in \Delta$ and $G \in \Gamma$. (E.g., if $\Gamma$ consists of a single point then $\Delta * \Gamma$ is the cone over $\Delta$. If $\Gamma$ consists of two disjoint points, then $\Delta * \Gamma$ is the suspension of $\Delta$.) We say that $\Delta$ and $\Gamma$ are joinfactors of $\Delta * \Gamma$. Now let $M$ be a matroid and $S \subset M$ a modular flat, i.e., $S$ is a modular element of $L_{M}$. Order the points of $M$ such that if $p \in S$ and $q \notin S$, then $p<q$. Show that $\mathrm{BC}(S)$ is a join-factor of $\mathrm{BC}(M)$. Deduce that $\chi_{M}(t)$ is divisible by $\chi_{S}(t)$.

(b) [2+] Conversely, let $M$ be a matroid and $S \subset M$. Label the points of $M$ so that if $p \in S$ and $q \notin S$, then $p<q$. Suppose that $\mathrm{BC}(S)$ is a join-factor of $\mathrm{BC}(M)$. Show that $S$ is modular.

(13) [2] Do Exercise 3.7, this time using Theorem 4.12 (the Broken Circuit Theorem).

(14) [1] Show that all geometric lattices of rank two are supersolvable.

(15) [2] Give an example of two nonisomorphic supersolvable geometric lattices of rank 3 with the same characteristic polynomials.

(16) [2] Prove Proposition 4.11: if $G$ is a graph with blocks $G_{1}, \ldots, G_{k}$, then $L_{G} \cong$ $L_{G_{1}} \times \cdots \times L_{G_{k}}$.

(17) $[2+]$ Give an example of a nonsupersolvable geometric lattice of rank three whose characteristic polynomial has only integer zeros.

(18) [2] Let $L_{1}$ and $L_{2}$ be geometric lattices. Show that $L_{1}$ and $L_{2}$ are supersolvable if and only if $L_{1} \times L_{2}$ is supersolvable.

(19) [3-] Let $L$ be a supersolvable geometric lattice. Show that every interval of $L$ is also supersolvable.

(20) [2] (a) Find the number of maximal chains of the partition lattice $\Pi_{n}$.

(b) Find the number of modular maximal chains of $\Pi_{n}$.

(21) $[2+]$ Show that the broken circuit complex of a matroid is pure (Corollary 4.6).

(22) Let $M$ be a matroid with a linear ordering of its points. The internal activity of a basis $B$ is the number of points $p \in B$ such that $p<q$ for all points $q \neq p$ not in the closure $\overline{B-p}$ of $B-p$. The external activity of $B$ is the number of points $p^{\prime} \in M-B$ such that $p^{\prime}<q^{\prime}$ for all $q^{\prime} \neq p^{\prime}$ contained in the unique circuit that is a subset of $B \cup\left\{p^{\prime}\right\}$. Define the Crapo beta invariant of $M$ by

$$
\beta(M)=(-1)^{\mathrm{rk}(M)-1} \chi_{M}^{\prime}(1),
$$

where' denotes differentiation.

(a) $[1+]$ Show that $1-\chi_{M}^{\prime}(1)=\psi\left(\mathrm{BC}_{r}\right)$, the Euler characteristic of the reduced broken circuit complex of $M$.

(b) [3-] Show that $\beta(M)$ is equal to the number of bases of $M$ with internal activity 0 and external activity 0 .

(c) [2] Let $\mathcal{A}$ be a real central arrangement with associated matroid $M_{\mathcal{A}}$. Suppose that $\mathcal{A}=c \mathcal{A}^{\prime}$ for some arrangement $\mathcal{A}^{\prime}$, where $c \mathcal{A}^{\prime}$ denotes the cone over $\mathcal{A}^{\prime}$. Show that $\beta\left(M_{\mathcal{A}}\right)=b\left(\mathcal{A}^{\prime}\right)$.

(d) [2+] With $\mathcal{A}$ as in (c), let $H^{\prime}$ be a (proper) translate of some hyperplane $H \in \mathcal{A}$. Show that $\beta\left(M_{\mathcal{A}}\right)=b\left(\mathcal{A} \cup\left\{H^{\prime}\right\}\right)$. 



\section{LECTURE 5 Finite fields}

\subsection{The finite field method}

In this lecture we will describe a method based on finite fields for computing the characteristic polynomial of an arrangement defined over $\mathbb{Q}$. We will then discuss several interesting examples. The main result (Theorem 5.15) is implicit in the work of Crapo and Rota $[\mathbf{1 3}, \S 17]$. It was first developed into a systematic tool for computing characteristic polynomials by Athanasiadis [1][2], after a closely related but not as general technique was presented by Blass and Sagan [9].

Suppose that the arrangement $\mathcal{A}$ is defined over $\mathbb{Q}$. By multiplying each hyperplane equation by a suitable integer, we may assume $\mathcal{A}$ is defined over $\mathbb{Z}$. In that case we can take coefficients modulo a prime $p$ and get an arrangement $\mathcal{A}_{q}$ defined over the finite field $\mathbb{F}_{q}$, where $q=p^{r}$. We say that $\mathcal{A}$ has good reduction mod $p$ (or over $\left.\mathbb{F}_{q}\right)$ if $L(\mathcal{A}) \cong L\left(\mathcal{A}_{q}\right)$.

For instance, let $\mathcal{A}$ be the affine arrangement in $\mathbb{Q}^{1}=\mathbb{Q}$ consisting of the points 0 and 10. Then $L(\mathcal{A})$ contains three elements, viz., $\mathbb{Q},\{0\}$, and $\{10\}$. If $p \neq 2,5$ then 0 and 10 remain distinct, so $\mathcal{A}$ has good reduction. On the other hand, if $p=2$ or $p=5$ then $0=10$ in $\mathbb{F}_{p}$, so $L\left(\mathcal{A}_{p}\right)$ contains just two elements. Hence $\mathcal{A}$ has bad reduction when $p=2,5$.

Proposition 5.13. Let $\mathcal{A}$ be an arrangement defined over $\mathbb{Z}$. Then $\mathcal{A}$ has good reduction for all but finitely many primes $p$.

Proof. Let $H_{1}, \ldots, H_{j}$ be affine hyperplanes, where $H_{i}$ is given by the equation $v_{i} \cdot x=a_{i}\left(v_{i}, a_{i} \in \mathbb{Z}^{n}\right)$. By linear algebra, we have $H_{1} \cap \cdots \cap H_{j} \neq \emptyset$ if and only if

$$
\operatorname{rank}\left[\begin{array}{cc}
v_{1} & a_{1} \\
\vdots & \vdots \\
v_{j} & a_{j}
\end{array}\right]=\operatorname{rank}\left[\begin{array}{c}
v_{1} \\
\vdots \\
v_{j}
\end{array}\right]
$$

Moreover, if (36) holds then

$$
\operatorname{dim}\left(H_{1} \cap \cdots \cap H_{j}\right)=n-\operatorname{rank}\left[\begin{array}{c}
v_{1} \\
\vdots \\
v_{j}
\end{array}\right] .
$$


Now for any $r \times s$ matrix $A$, we have $\operatorname{rank}(A) \geq t$ if and only if some $t \times t$ submatrix $B$ satisfies $\operatorname{det}(B) \neq 0$. It follows that $L(\mathcal{A}) \neq L\left(\mathcal{A}_{p}\right)$ if and only if at least one member $S$ of a certain finite collection $\mathcal{S}$ of subsets of integer matrices $B$ satisfies the following condition:

$$
(\forall B \in S) \operatorname{det}(B) \neq 0 \text { but } \operatorname{det}(B) \equiv 0(\bmod p) .
$$

This can only happen for finitely many $p$, viz., for certain $B$ we must have $p \mid \operatorname{det}(B)$, so $L(\mathcal{A}) \cong L\left(\mathcal{A}_{p}\right)$ for $p$ sufficiently large.

The main result of this section is the following. Like many fundamental results in combinatorics, the proof is easy but the applicability very broad.

Theorem 5.15. Let $\mathcal{A}$ be an arrangement in $\mathbb{Q}^{n}$, and suppose that $L(\mathcal{A}) \cong L\left(\mathcal{A}_{q}\right)$ for some prime power $q$. Then

$$
\begin{aligned}
\chi_{\mathcal{A}}(q) & =\#\left(\mathbb{F}_{q}^{n}-\bigcup_{H \in \mathcal{A}_{q}} H\right) \\
& =q^{n}-\# \bigcup_{H \in \mathcal{A}_{q}} H .
\end{aligned}
$$

Proof. Let $x \in L\left(\mathcal{A}_{q}\right)$ so $\# x=q^{\operatorname{dim}(x)}$. Here $\operatorname{dim}(x)$ can be computed either over $\mathbb{Q}$ or $F_{q}$. Define two functions $f, g: L\left(\mathcal{A}_{q}\right) \rightarrow \mathbb{Z}$ by

$$
\begin{aligned}
& f(x)=\# x \\
& g(x)=\#\left(x-\bigcup_{y>x} y\right) .
\end{aligned}
$$

In particular,

$$
g(\hat{0})=g\left(\mathbb{F}_{q}^{n}\right)=\#\left(\mathbb{F}_{q}^{n}-\bigcup_{H \in \mathcal{A}_{q}} H\right)
$$

Clearly

$$
f(x)=\sum_{y \geq x} g(y) .
$$

Let $\mu$ denote the Möbius function of $L(\mathcal{A}) \cong L\left(\mathcal{A}_{q}\right)$. By Möbius inversion (Theorem 1.1),

$$
\begin{aligned}
g(x) & =\sum_{y \geq x} \mu(x, y) f(y) \\
& =\sum_{y \geq x} \mu(x, y) q^{\operatorname{dim}(y)} .
\end{aligned}
$$

Put $x=\hat{0}$ to get

$$
g(\hat{0})=\sum_{y} \mu(y) q^{\operatorname{dim}(y)}=\chi_{\mathcal{A}}(q) .
$$

For the remainder of this lecture, we will be concerned with applications of Theorem 5.15 and further interesting examples of arrangements. 
Example 5.12. Let $G$ be a graph with vertices $1,2, \ldots, n$, so

$$
Q_{\mathcal{A}_{G}}(x)=\prod_{i j \in E(G)}\left(x_{i}-x_{j}\right) .
$$

Then by Theorem 5.15,

$$
\begin{aligned}
\chi_{\mathcal{A}_{G}}(q) & =q^{n}-\#\left\{\left(\alpha_{1}, \ldots, \alpha_{n}\right) \in \mathbb{F}_{1}^{n}: \alpha_{i}=\alpha_{j} \text { for some } i j \in E(G)\right\} \\
& =\#\left\{\left(\beta_{1}, \ldots, \beta_{n}\right) \in \mathbb{F}_{q}^{n}: \beta_{i} \neq \beta_{j} \forall i j \in E(G)\right\} \\
& =\chi_{G}(q),
\end{aligned}
$$

in agreement with Theorem 2.7. Note that this equality holds for all prime powers $q$, not just for $p^{m}$ with $p \gg 0$. This is because the matrix with rows $e_{i}-e_{j}$, where $i j \in E(G)$ and $e_{i}$ is the $i$ th unit coordinate vector in $\mathbb{Q}^{n}$, is totally unimodular, i.e., every minor (determinant of a square submatrix) is $0, \pm 1$. Hence the nonvanishing of a minor is independent of the ambient field.

A very interesting class of arrangements, including the braid arrangement, is associated with root systems, or more generally, finite reflection groups. We will simply mention some basic results here without proof. A root system is a finite set $R$ of nonzero vectors in $\mathbb{R}^{n}$ satisfying certain properties that we will not give here. (References include $[\mathbf{6}][\mathbf{1 0}][\mathbf{2 0}]$.) The Coxeter arrangement $\mathcal{A}(R)$ consists of the hyperplanes $\alpha \cdot x=0$, where $\alpha \in R$. There are four infinite (irreducible) classes of root systems (all in $\mathbb{R}^{n}$ ):

$$
\begin{aligned}
A_{n-1} & =\left\{e_{i}-e_{j}: 1 \leq i<j \leq n\right\}=\mathcal{B}_{n} \\
D_{n} & =\left\{e_{i}-e_{j}, e_{i}+e_{j}: 1 \leq i<j \leq n\right\} \\
B_{n} & =D_{n} \cup\left\{e_{i}: 1 \leq i \leq n\right\} \\
C_{n} & =D_{n} \cup\left\{2 e_{i}: 1 \leq i \leq n\right\} .
\end{aligned}
$$

We should really regard $A_{n-1}$ as being a subset of the space

$$
\left\{\left(\alpha_{1}, \ldots, \alpha_{n}\right) \in \mathbb{R}^{n}: \sum \alpha_{i}=0\right\} \cong \mathbb{R}^{n-1} .
$$

We thus obtain the following Coxeter arrangements. In all cases $1 \leq i<j \leq n$ and $1 \leq k \leq n$.

$$
\begin{aligned}
\mathcal{A}\left(A_{n-1}\right)=\mathcal{B}_{n} & : \quad x_{i}-x_{j}=0 \\
\mathcal{A}\left(B_{n}\right)=\mathcal{A}\left(C_{n}\right) & : \quad x_{i}-x_{j}=0, x_{i}+x_{j}=0, x_{k}=0 \\
\mathcal{A}\left(D_{n}\right) & : \quad x_{i}-x_{j}=0, x_{i}+x_{j}=0 .
\end{aligned}
$$

See Figure 1 for the arrangements $\mathcal{A}\left(B_{2}\right)$ and $\mathcal{A}\left(D_{2}\right)$.

Let us compute the characteristic polynomial $\chi_{\mathcal{A}\left(B_{n}\right)}(q)$. For $p \gg 0$ (actually $p>2$ ) and $q=p^{m}$ we have

$$
\chi_{\mathcal{A}\left(B_{n}\right)}(q)=\#\left\{\left(\alpha_{1}, \ldots, \alpha_{n}\right) \in \mathbb{F}_{q}^{n}: \alpha_{i} \neq \pm \alpha_{j}(i \neq j), \alpha_{i} \neq 0(1 \leq i \leq n)\right\} .
$$

Choose $\alpha_{1} \in \mathbb{F}_{q}^{*}=\mathbb{F}_{q}-\{0\}$ in $q-1$ ways. Then choose $\alpha_{2} \in \mathbb{F}_{q}^{*}-\left\{\alpha_{1},-\alpha_{1}\right\}$ in $q-3$ ways, then $\alpha_{3}$ in $q-5$ ways, etc., to obtain:

$$
\chi_{\mathcal{A}\left(B_{n}\right)}(t)=(t-1)(t-3) \cdots(t-(2 n-1)) .
$$

In particular,

$$
r\left(\mathcal{A}\left(B_{n}\right)\right)=(-1)^{n} \chi_{\mathcal{A}\left(B_{n}\right)}(-1)=2 \cdot 4 \cdot 6 \cdots(2 n)=2^{n} n ! .
$$




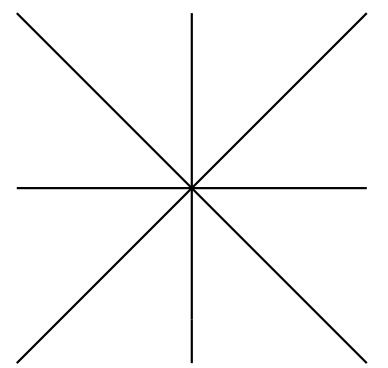

$A\left(B_{2}\right)$

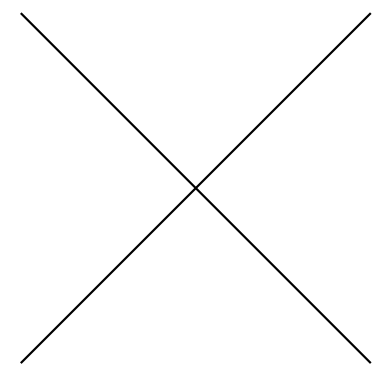

$A\left(D_{2}\right)$

Figure 1. The arrangements $\mathcal{A}\left(B_{2}\right)$ and $\mathcal{A}\left(D_{2}\right)$

By a similar but slightly more complicated argument we get (Exercise 1)

$$
\chi_{\mathcal{A}\left(D_{n}\right)}(t)=(t-1)(t-3) \cdots(t-(2 n-3)) \cdot(t-n+1) .
$$

Note. Coxeter arrangements are always free in the sense of Theorem 4.14 (a result of Terao [34]), but need not be supersolvable. In fact, $\mathcal{A}\left(A_{n}\right)$ and $\mathcal{A}\left(B_{n}\right)$ are supersolvable, but $\mathcal{A}\left(D_{n}\right)$ is not supersolvable for $n \geq 4$ [4, Thm. 5.1].

\subsection{The Shi arrangement}

We next consider a modification (or deformation) of the braid arrangement called the Shi arrangement $[\mathbf{2 7}, \S 7]$ and denoted $\mathcal{S}_{n}$. It consists of the hyperplanes

$$
x_{i}-x_{j}=0,1, \quad 1 \leq i<j \leq n .
$$

Thus $\mathcal{S}_{n}$ has $n(n-1)$ hyperplanes and $\operatorname{rank}\left(\mathcal{S}_{n}\right)=n-1$. Figure 2 shows the Shi arrangement $\mathcal{S}_{3}$ in $\operatorname{ker}\left(x_{1}+x_{2}+x_{3}\right) \cong \mathbb{R}^{2}$ (i.e., the space $\left\{\left(x_{1}, x_{2}, x_{3}\right) \in \mathbb{R}^{3}\right.$ : $\left.\left.x_{1}+x_{2}+x_{3}=0\right\}\right)$.

Theorem 5.16. The characteristic polynomial of $\mathcal{S}_{n}$ is given by

$$
\chi_{s_{n}}(t)=t(t-n)^{n-1} \text {. }
$$

Proof. Let $p$ be a large prime. By Theorem 5.15 we have

$$
\chi_{\mathfrak{S}_{n}}(p)=\#\left\{\left(\alpha_{1}, \ldots, \alpha_{n}\right) \in \mathbb{F}_{p}^{n}: i<j \Rightarrow \alpha_{i} \neq \alpha_{j} \text { and } \alpha_{i} \neq \alpha_{j}+1\right\} .
$$

Choose a weak ordered partition $\pi=\left(B_{1}, \ldots, B_{p-n}\right)$ of $[n]$ into $p-n$ blocks, i.e., $\bigcup B_{i}=[n]$ and $B_{i} \cap B_{j}=\emptyset$ if $i \neq j$, such that $1 \in B_{1}$. ("Weak" means that we allow $B_{i}=\emptyset$.) For $2 \leq i \leq n$ there are $p-n$ choices for $j$ such that $i \in B_{j}$, so $(p-n)^{n-1}$ choices in all. We will illustrate the following argument with the example $p=11, n=6$, and

$$
\pi=(\{1,4\},\{5\}, \emptyset,\{2,3,6\}, \emptyset) .
$$

Arrange the elements of $\mathbb{F}_{p}$ clockwise on a circle. Place $1,2, \ldots, n$ on some $n$ of these points as follows. Place elements of $B_{1}$ consecutively (clockwise) in increasing order with 1 placed at some element $\alpha_{1} \in \mathbb{F}_{p}$. Skip a space and place the elements of $B_{2}$ consecutively in increasing order. Skip another space and place the elements of $B_{3}$ consecutively in increasing order, etc. For our example (38), say $\alpha_{1}=6$. 


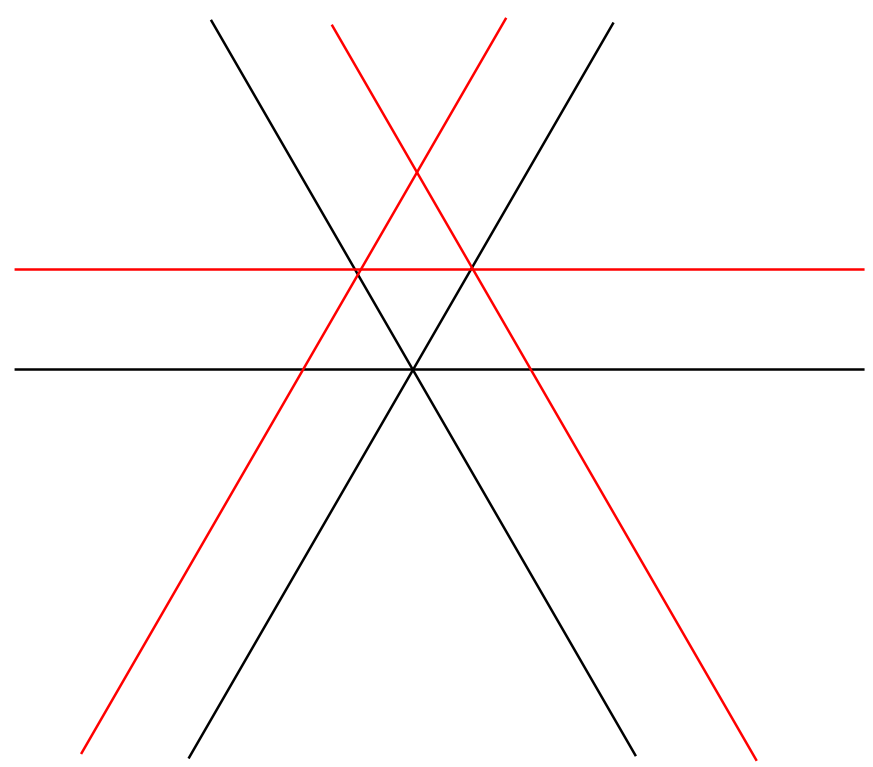

Figure 2. The Shi arrangement $\delta_{3}$ in $\operatorname{ker}\left(x_{1}+x_{2}+x_{3}\right)$

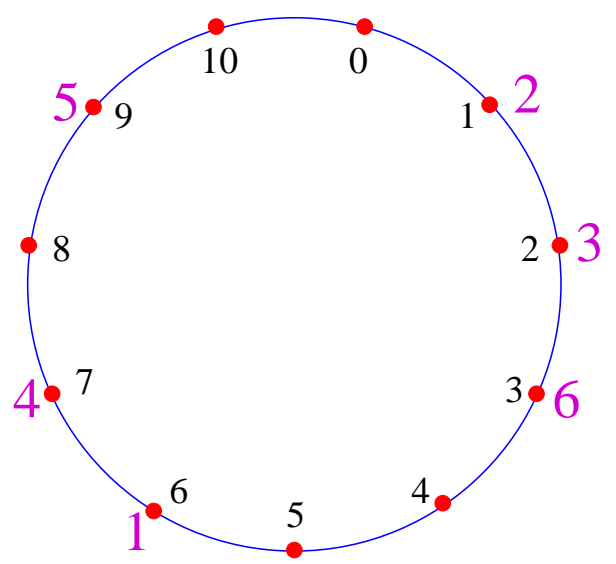

Let $\alpha_{i}$ be the position (element of $\mathbb{F}_{p}$ ) at which $i$ was placed. For our example we have

$$
\left(\alpha_{1}, \alpha_{2}, \alpha_{3}, \alpha_{4}, \alpha_{5}, \alpha_{6}\right)=(6,1,2,7,9,3) .
$$

It is easily verified that we have defined a bijection from the $(p-n)^{n-1}$ weak ordered partitions $\pi=\left(B_{1}, \ldots, B_{p-n}\right)$ of $[n]$ into $p-n$ blocks such that $1 \in B_{1}$, together with the choice of $\alpha_{1} \in \mathbb{F}_{p}$, to the set $\mathbb{F}_{p}^{n}-\cup_{H \in\left(\mathcal{S}_{n}\right)_{p}} H$. There are $(p-n)^{n-1}$ choices for $\pi$ and $p$ choices for $\alpha_{1}$, so it follows from Theorem 5.15 that $\chi_{\mathbb{S}_{n}}(t)=t(t-n)^{n-1}$.

We obtain the following corollary immediately from Theorem 2.5.

Corollary 5.11. We have $r\left(\mathcal{S}_{n}\right)=(n+1)^{n-1}$ and $b\left(\mathcal{S}_{n}\right)=(n-1)^{n-1}$.

Note. Since $r\left(\mathcal{S}_{n}\right)$ and $b\left(\mathcal{S}_{n}\right)$ have such simple formulas, it is natural to ask for a direct bijective proof of Corollary 5.11. A number of such proofs are known; a sketch that $r\left(S_{n}\right)=(n+1)^{n-1}$ is given in Exercise 3 . 
Note. It can be shown that the cone $c \mathcal{S}_{n}$ is not supersolvable for $n \geq 3$ (Exercise 4) but is free in the sense of Theorem 4.14.

\subsection{Exponential sequences of arrangements}

The braid arrangement (in fact, any Coxeter arrangement) is highly symmetrical; indeed, the group of linear transformations that preserves the arrangement acts transitively on the regions. Thus all regions "look the same." The Shi arrangement lacks this symmetry, but it still possesses a kind of "combinatorial symmetry" that allows us to express the characteristic polynomials $\chi_{\mathcal{S}_{n}}(t)$, for all $n \geq 1$, in terms of the number $r\left(S_{n}\right)$ of regions.

Definition 5.14. A sequence $\mathfrak{A}=\left(\mathcal{A}_{1}, \mathcal{A}_{2}, \ldots\right)$ of arrangements is called an exponential sequence of arrangements (ESA) if it satisfies the following three conditions.

(1) $\mathcal{A}_{n}$ is in $K^{n}$ for some field $K$ (independent of $n$ ).

(2) Every $H \in \mathcal{A}_{n}$ is parallel to some hyperplane $H^{\prime}$ in the braid arrangement $\mathcal{B}_{n}($ over $K)$.

(3) Let $S$ be a $k$-element subset of $[n]$, and define

$\mathcal{A}_{n}^{S}=\left\{H \in \mathcal{A}_{n}: H\right.$ is parallel to $x_{i}-x_{j}=0$ for some $\left.i, j \in S\right\}$.

Then $L\left(\mathcal{A}_{n}^{S}\right) \cong L\left(\mathcal{A}_{k}\right)$.

Examples of ESA's are given by $\mathcal{A}_{n}=\mathcal{B}_{n}$ or $\mathcal{A}_{n}=\mathcal{S}_{n}$. In fact, in these cases we have $\mathcal{A}_{n}^{S} \cong \mathcal{A}_{k} \times K^{n-k}$.

The combinatorial properties of ESA's are related to the exponential formula in the theory of exponential generating functions $[32, \S 5.1]$, which we now review. Informally, we are dealing with "structures" that can be put on a vertex set $V$ such that each structure is a disjoint union of its "connected components." We obtain a structure on $V$ by partitioning $V$ and placing a connected structure on each block (independently). Examples of such structures are graphs, forests, and posets, but not trees or groups. Let $h(n)$ be the total number of structures on an $n$-set $V$ (with $h(0)=1$ ), and let $f(n)$ be the number that are connected. The exponential formula states that

$$
\sum_{n \geq 0} h(n) \frac{x^{n}}{n !}=\exp \sum_{n \geq 1} f(n) \frac{x^{n}}{n !} .
$$

More precisely, let $f: \mathbb{P} \rightarrow R$, where $R$ is a commutative ring. (For our purposes, $R=\mathbb{Z}$ will do.) Define a new function $h: \mathbb{N} \rightarrow R$ by $h(0)=1$ and

$$
h(n)=\sum_{\pi=\left\{B_{1}, \ldots, B_{k}\right\} \in \Pi_{n}} f\left(\# B_{1}\right) f\left(\# B_{2}\right) \cdots f\left(\# B_{k}\right) .
$$

Then equation (39) holds. A straightforward proof can be given by considering the expansion

$$
\begin{aligned}
\exp \sum_{n \geq 1} f(n) \frac{x^{n}}{n !} & =\prod_{n \geq 1} \exp f(n) \frac{x^{n}}{n !} \\
& =\prod_{n \geq 1}\left(\sum_{k \geq 0} f(n)^{k} \frac{x^{k n}}{n !^{k} k !}\right) .
\end{aligned}
$$

We omit the details (Exercise 5). 
For any arrangement $\mathcal{A}$ in $K^{n}$, define $r(\mathcal{A})=(-1)^{n} \chi_{\mathcal{A}}(-1)$. Of course if $K=\mathbb{R}$ this coincides with the definition of $r(\mathcal{A})$ as the number of regions of $\mathcal{A}$. We come to the main result concering ESA's.

Theorem 5.17. Let $\mathfrak{A}=\left(\mathcal{A}_{1}, \mathcal{A}_{2}, \ldots\right)$ be an ESA. Then

$$
\sum_{n \geq 0} \chi_{\mathcal{A}_{n}}(t) \frac{x^{n}}{n !}=\left(\sum_{n \geq 0}(-1)^{n} r\left(\mathcal{A}_{n}\right) \frac{x^{n}}{n !}\right)^{-t} .
$$

Example 5.13. For $\mathfrak{A}=\left(\mathcal{B}_{1}, \mathcal{B}_{2}, \ldots\right)$ Theorem 5.17 asserts that

$$
\sum_{n \geq 0} t(t-1) \cdots(t-n+1) \frac{x^{n}}{n !}=\left(\sum_{n \geq 0}(-1)^{n} n ! \frac{x^{n}}{n !}\right)^{-t}
$$

as immediately follows from the binomial theorem. On the other hand, if $\mathfrak{A}=$ $\left(S_{1}, S_{2}, \ldots\right)$, then we obtain the much less obvious identity

$$
\sum_{n \geq 0} t(t-n)^{n-1} \frac{x^{n}}{n !}=\left(\sum_{n \geq 0}(-1)^{n}(n+1)^{n-1} \frac{x^{n}}{n !}\right)^{-t} .
$$

Proof of Theorem 5.17. By Whitney's theorem (Theorem 2.4) we have for any arrangement $\mathcal{A}$ in $K^{n}$ that

$$
\chi_{\mathcal{A}}(t)=\sum_{\substack{\mathcal{B} \subseteq \mathcal{A} \\ \mathcal{B} \text { central }}}(-1)^{\# \mathcal{B}} t^{n-\operatorname{rank}(\mathcal{B})} .
$$

Let $\mathfrak{A}=\left(\mathcal{A}_{1}, \mathcal{A}_{2}, \ldots\right)$, and let $\mathcal{B} \subseteq \mathcal{A}_{n}$ for some $n$. Define $\pi(\mathcal{B}) \in \Pi_{n}$ to have blocks that are the vertex sets of the connected components of the graph $G$ on $[n]$ with edges

$$
E(G)=\left\{i j: \exists x_{i}-x_{j}=c \text { in } \mathcal{B}\right\} .
$$

Define

$$
\tilde{\chi}_{\mathcal{A}_{n}}(t)=\sum_{\substack{\mathcal{B} \subseteq \mathcal{A} \\ \mathcal{B} \text { central } \\ \pi(\mathcal{B})=[n]}}(-1)^{\# \mathcal{B}} t^{n-\operatorname{rk}(\mathcal{B})}
$$

Then

$$
\begin{aligned}
\chi_{\mathcal{A}_{n}}(t) & =\sum_{\pi=\left\{B_{1}, \ldots, B_{k}\right\} \in \Pi_{n}} \sum_{\substack{\mathcal{B} \subseteq \mathcal{A} \\
\boldsymbol{B} \text { central } \\
\pi(\mathcal{B})=\pi}}(-1)^{\# \mathcal{B}} t^{n-\mathrm{rk}(\mathcal{B})} \\
& =\sum_{\pi=\left\{B_{1}, \ldots, B_{k}\right\} \in \Pi_{n}} \tilde{\chi}_{\mathcal{A}_{\# B_{1}}}(t) \tilde{\chi}_{\mathcal{A}_{\# B_{2}}}(t) \cdots \tilde{\chi}_{\mathcal{A}_{\# B_{k}}}(t) .
\end{aligned}
$$

Thus by the exponential formula (39),

$$
\sum_{n \geq 0} \chi_{\mathcal{A}_{n}}(t) \frac{x^{n}}{n !}=\exp \sum_{n \geq 1} \tilde{\chi}_{\mathcal{A}_{n}}(t) \frac{x^{n}}{n !} .
$$


But $\pi(\mathcal{B})=[n]$ if and only if $\operatorname{rk}(\mathcal{B})=n-1$, so $\tilde{\chi}_{\mathcal{A}_{n}}(t)=c_{n} t$ for some $c_{n} \in \mathbb{Z}$. We therefore get

$$
\begin{aligned}
\sum_{n \geq 0} \chi_{\mathcal{A}_{n}}(t) \frac{x^{n}}{n !} & =\exp t \sum_{n \geq 1} c_{n} \frac{x^{n}}{n !} \\
& =\left(\sum_{n \geq 0} b_{n} \frac{x^{n}}{n !}\right)^{t},
\end{aligned}
$$

where $\exp \sum_{n \geq 1} c_{n} \frac{x^{n}}{n !}=\sum_{n \geq 0} b_{n} \frac{x^{n}}{n !}$. Put $t=-1$ to get

$$
\sum_{n \geq 0}(-1)^{n} r\left(\mathcal{A}_{n}\right) \frac{x^{n}}{n !}=\left(\sum_{n \geq 0} b_{n} \frac{x^{n}}{n !}\right)^{-1},
$$

from which it follows that

$$
\sum_{n \geq 0} \chi_{\mathcal{A}_{n}}(t) \frac{x^{n}}{n !}=\left(\sum_{n \geq 0}(-1)^{n} r\left(\mathcal{A}_{n}\right) \frac{x^{n}}{n !}\right)^{-t} .
$$

For a generalization of Theorem 5.17, see Exercise 10.

\subsection{The Catalan arrangement}

Define the Catalan arrangement $\mathcal{C}_{n}$ in $K^{n}$, where $\operatorname{char}(K) \neq 2$, by

$$
Q_{\mathfrak{C}_{n}}(x)=\prod_{1 \leq i<j \leq n}\left(x_{i}-x_{j}\right)\left(x_{i}-x_{j}-1\right)\left(x_{i}-x_{j}+1\right) .
$$

Equivalently, the hyperplanes of $\mathcal{C}_{n}$ are given by

$$
x_{i}-x_{j}=-1,0,1, \quad 1 \leq i<j \leq n .
$$

Thus $\mathcal{C}_{n}$ has $3\left(\begin{array}{l}n \\ 2\end{array}\right)$ hyperplanes, and $\operatorname{rank}\left(\mathcal{C}_{n}\right)=n-1$.

Assume now that $K=\mathbb{R}$. The symmetric group $\mathfrak{S}_{n}$ acts on $\mathbb{R}^{n}$ by permuting coordinates, i.e.,

$$
w \cdot\left(x_{1}, \ldots, x_{n}\right)=\left(x_{w(1)}, \ldots, x_{w(n)}\right) .
$$

Here we are multiplying permutations left-to-right, e.g., $(1,2)(2,3)=(1,3,2)$ (in cycle form), so $v w \cdot \alpha=v \cdot(w \cdot \alpha)$. Both $\mathcal{B}_{n}$ and $\mathcal{C}_{n}$ are $\mathfrak{S}_{n}$-invariant, i.e., $\mathfrak{S}_{n}$ permutes the hyperplanes of these arrangements. Hence $\mathfrak{S}_{n}$ also permutes their regions, and each region $x_{w(1)}>x_{w(2)}>\cdots>x_{w(n)}$ of $\mathcal{B}_{n}$ is divided "in the same way" in $\mathcal{C}_{n}$. In particular, if $r_{0}\left(\mathcal{C}_{n}\right)$ denotes the number of regions of $\mathcal{C}_{n}$ contained in some fixed region of $\mathcal{B}_{n}$, then $r\left(\mathcal{C}_{n}\right)=n ! r_{0}\left(\mathcal{C}_{n}\right)$. See Figure 3 for $\mathcal{C}_{3}$ in the ambient space $\operatorname{ker}\left(x_{1}+x_{2}+x_{3}\right)$, where the hyperplanes of $\mathcal{B}_{3}$ are drawn as solid lines and the remaining hyperplanes as dashed lines. Each region of $\mathcal{B}_{3}$ contains five regions of $\mathcal{C}_{3}$, so $r\left(\mathcal{C}_{3}\right)=6 \cdot 5=30$.

We can compute $r\left(\mathcal{C}_{n}\right)$ (or equivalently $r_{0}\left(\mathcal{C}_{n}\right)$ ) by a direct combinatorial argument. Let $R_{0}$ denote the region $x_{1}>x_{2}>\cdots>x_{n}$ of $\mathcal{B}_{n}$. The regions of $\mathcal{C}_{n}$ contained in $R_{0}$ are determined by those $i<j$ such that $x_{i}-x_{j}<1$. We need only specify the maximal intervals $[i, j]$ such that $x_{i}-x_{j}<1$, i.e., if $a \leq i<j \leq b$ and $x_{a}-x_{b}<1$, then $a=i$ and $b=j$. It is easy to see that any such specification of maximal intervals determines a region of $\mathcal{C}_{n}$ contained in $R_{0}$. Thus $r_{0}\left(\mathcal{C}_{n}\right)$ is equal 


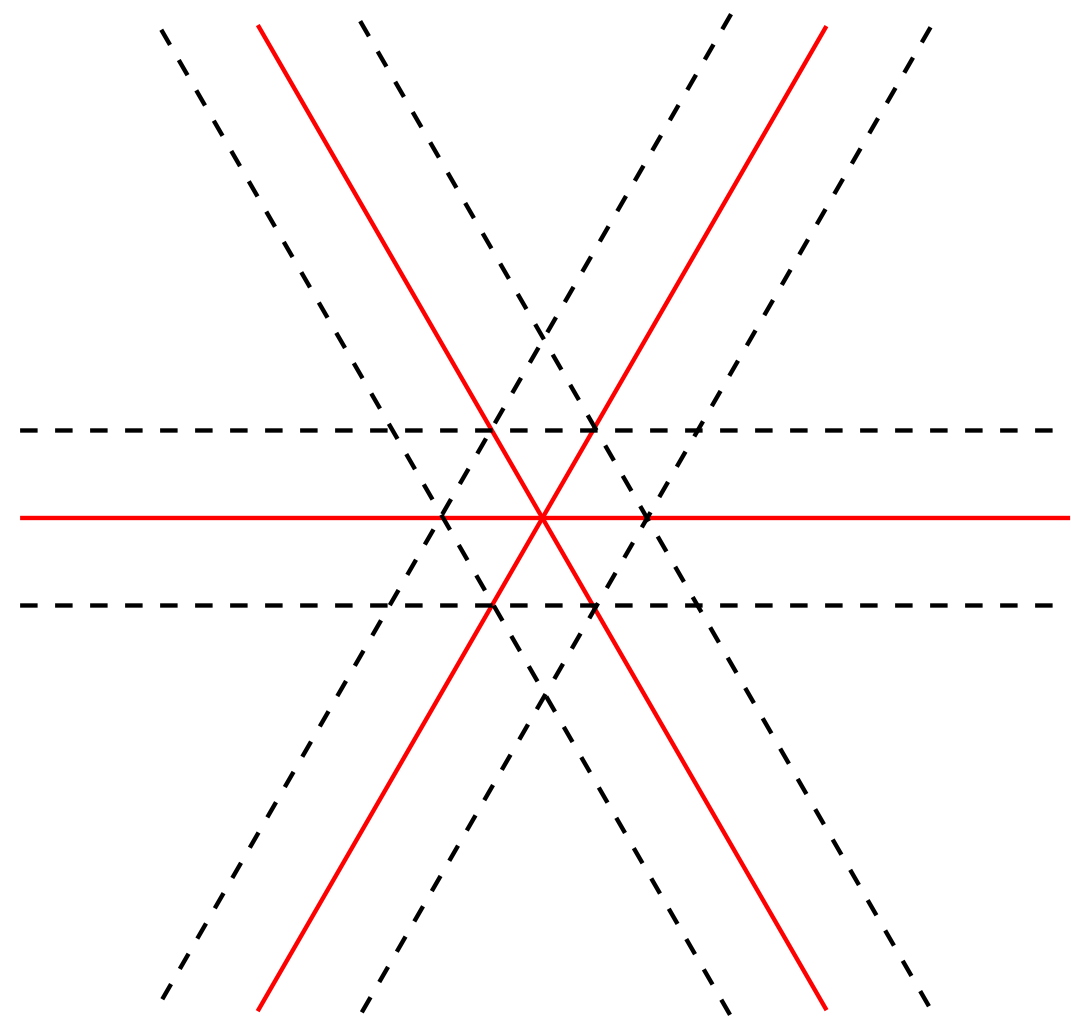

Figure 3. The Catalan arrangement $\mathcal{C}_{3}$ in $\operatorname{ker}\left(x_{1}+x_{2}+x_{3}\right)$

to the number of antichains $A$ of strict intervals of $[n]$, i.e., sets $A$ of intervals $[i, j]$, where $1 \leq i<j \leq n$, such that no interval in $A$ is contained in another. ("Strict" means that $i=j$ is not allowed.) It is known (equivalent to [32, Exer. 6.19(bbb)]) that the number of such antichains is the Catalan number $C_{n}=\frac{1}{n+1}\left(\begin{array}{c}2 n \\ n\end{array}\right)$. For the sake of completeness we give a bijection between these antichains and a standard combinatorial structure counted by Catalan numbers, viz., lattice paths from $(0,0)$ to $(n, n)$ with steps $(1,0)$ and $(0,1)$, never rising above the line $y=x([\mathbf{3 2}$, Exer. 6.19(h)]). Given an antichain $A$ of intervals of $[n]$, there is a unique lattice path of the claimed type whose "outer corners" (a step $(1,0)$ followed by $(0,1)$ ) consist of the points $(j, i-1)$ where $[i, j] \in A$, together with the points $(i, i-1)$ where no interval in $A$ contains $i$. Figure 4 illustrates this bijection for $n=8$ and $A=\{[1,4],[3,5],[7,8]\}$.

We have therefore proved the following result. For a refinement, see Exercise 11.

Proposition 5.14. The number of regions of the Catalan arrangement $\mathcal{C}_{n}$ is given by $r\left(\mathcal{C}_{n}\right)=n ! C_{n}$. Each region of $\mathcal{B}_{n}$ contains $C_{n}$ regions of $\mathcal{C}_{n}$.

In fact, there is a simple formula for the characteristic polynomial $\chi \mathrm{e}_{n}(t)$.

Theorem 5.18. We have

$$
\chi \mathrm{e}_{n}(t)=t(t-n-1)(t-n-2)(t-n-3) \cdots(t-2 n+1) .
$$




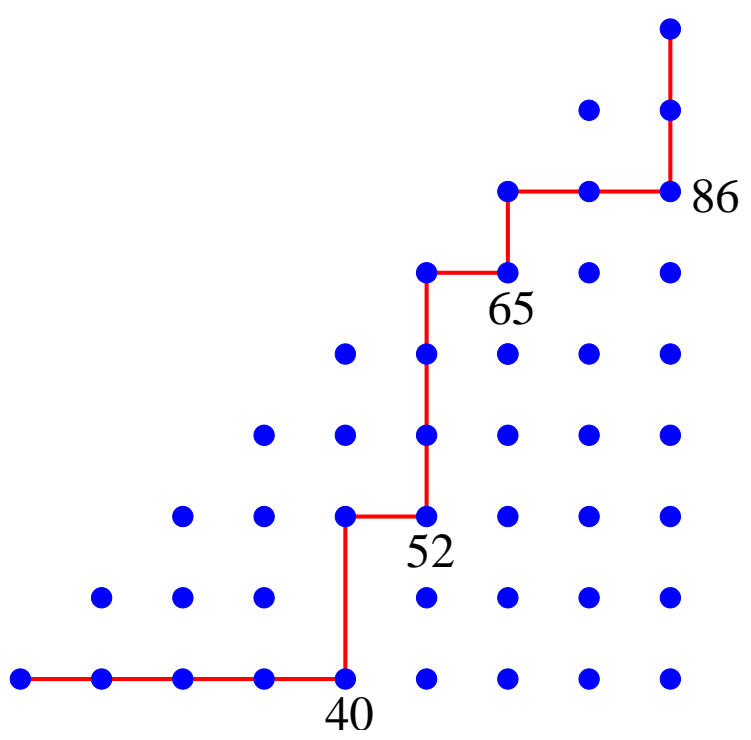

Figure 4. A bijection corresponding to $A=\{[1,4],[3,5],[7,8]\}$

Proof. Clearly the sequence $\left(\mathcal{C}_{1}, \mathcal{C}_{2}, \ldots\right)$ is an ESA, so by Theorem 5.17 we have

$$
\begin{aligned}
\sum_{n \geq 0} \chi_{\mathfrak{e}_{n}}(t) \frac{x^{n}}{n !} & =\left(\sum_{n \geq 0}(-1)^{n} n ! C_{n} \frac{x^{n}}{n !}\right)^{-t} \\
& =\left(\sum_{n \geq 0}(-1)^{n} C_{n} x^{n}\right)^{-t} .
\end{aligned}
$$

One method for expanding this series is to use the Lagrange inversion formula [32, Thm. 5.4.2]. Let $F(x)=a_{1} x+a_{2} x^{2}+\cdots$ be a formal power series over $K$, where $\operatorname{char}(K)=0$ and $a_{1} \neq 0$. Then there exists a unique formal power series $F^{\langle-1\rangle}=a_{1}^{-1} x+\cdots$ satisfying

$$
F\left(F^{\langle-1\rangle}(x)\right)=F^{\langle-1\rangle}(F(x))=x .
$$

Let $k, t \in \mathbb{Z}$. The Lagrange inversion formula states that

$$
t\left[x^{t}\right] F^{\langle-1\rangle}(x)^{k}=k\left[x^{t-k}\right]\left(\frac{x}{F(x)}\right)^{t} .
$$

Let $y=\sum_{n \geq 0}(-1)^{n} C_{n} x^{n+1}$. By a fundamental property of Catalan numbers, $y^{2}=-y+x$. Hence $y=\left(x+x^{2}\right)^{\langle-1\rangle}$. Substitute $t-n$ for $k$ and apply equation (42) to $y=F(x)$, so $F^{\langle-1\rangle}(x)=x+x^{2}$ :

$$
t\left[x^{t}\right]\left(x+x^{2}\right)^{t-n}=(t-n)\left[x^{n}\right]\left(\frac{x}{y}\right)^{t} .
$$

The right-hand side of (43) is just

$$
(t-n)\left[x^{n}\right]\left(\frac{y}{x}\right)^{-t}=\frac{(t-n) \chi_{\mathfrak{e}_{n}}(t)}{n !} .
$$



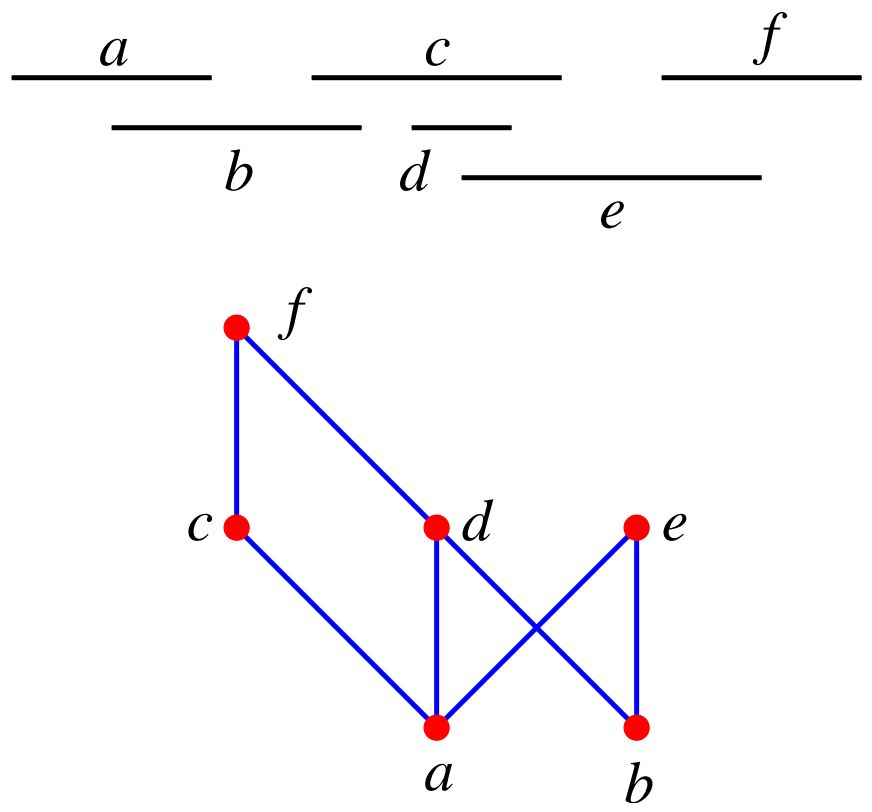

Figure 5. An example of an interval order

The left-hand side of (43) is given by

$$
t\left[x^{t}\right] x^{t-n}(1+x)^{t-n}=t\left(\begin{array}{c}
t-n \\
n
\end{array}\right)=\frac{t(t-n)(t-n-1) \cdots(t-2 n+1)}{n !} .
$$

It follows that

$$
\chi_{\mathfrak{e}_{n}}(t)=t(t-n-1)(t-n-2)(t-n-3) \cdots(t-2 n+1)
$$

for all $t \in \mathbb{Z}$. It then follows easily (e.g., using the fact that a polynomial in one variable over a field of characteristic 0 is determined by its values on $\mathbb{Z}$ ) that this equation holds when $t$ is an indeterminate.

NoTE. It is not difficult to give an alternative proof of Theorem 5.18 based on the finite field method (Exercise 12).

\subsection{Interval orders}

The subject of interval orders has a long history (see [15][36]), but only recently [33] was their connection with arrangements noticed. Let $P=\left\{I_{1}, \ldots, I_{n}\right\}$ be a finite set of closed intervals $I_{i}=\left[a_{i}, b_{i}\right]$, where $a_{i}, b_{i} \in \mathbb{R}$ and $a_{i}<b_{i}$. Partially order $P$ by defining $I_{i}<I_{j}$ if $b_{i}<a_{j}$, i.e., $I_{i}$ lies entirely to the left of $I_{j}$ on the real number line. A poset isomorphic to $P$ is called an interval order. Figure 5 gives an example of six intervals and the corresponding interval order. It is understood that the real line lies below and parallel to the line segments labelled $a, \ldots, f$, and that the actual intervals are the projections of these line segments to $\mathbb{R}$. If all the intervals $I_{i}$ have length one, then $P$ is called a semiorder or unit interval order.

We will be considering both labelled and unlabelled interval orders. A labelled interval order is the same as an interval order on a set $S$, often taken to be $[n]$. If an interval order $P$ corresponds to intervals $I_{1}, \ldots, I_{n}$, then there is a natural 


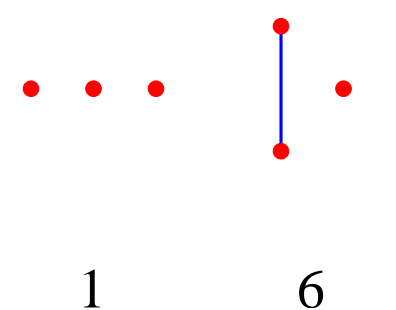

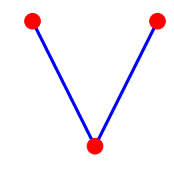

3

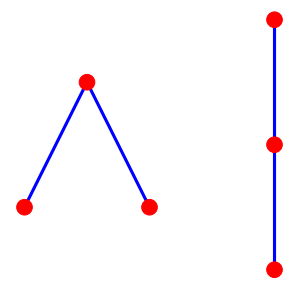

3

Figure 6. The number of labelings of semiorders with three elements

labeling of $P$, viz., label the element corresponding to $I_{i}$ by $i$. Thus the intervals $I_{1}=[0,1]$ and $I_{2}=[2,3]$ correspond to the labelled interval order $P_{1}$ defined by $1<2$, while the intervals $I_{1}=[2,3]$ and $I_{2}=[0,1]$ correspond to $P_{2}$ defined by $2<1$. Note that $P_{1}$ and $P_{2}$ are different labelled interval orders but are isomorphic as posets. As another example, consider the intervals $I_{1}=[0,2]$ and $I_{2}=[1,3]$. The corresponding labelled interval order $P$ consists of the disjoint points 1 and 2 . If we now let $I_{1}=[1,3]$ and $I_{2}=[0,2]$, then we obtain the same labelled interval order (or labelled poset) $P$, although the intervals themselves have been exchanged. An unlabelled interval order may be regarded as an isomorphism class of interval orders; two intervals orders $P_{1}$ and $P_{2}$ represent the same unlabelled interval order if and only if they are isomorphic. Of course our discussion of labelled and unlabelled interval orders applies equally well to semiorders.

Figure 6 shows the five nonisomorphic (or unlabelled) interval orders (which for three vertices coincides with semiorders) with three vertices, and below them the number of distinct labelings. (In general, the number of labelings of an $n$ element poset $P$ is $n ! / \# \operatorname{Aut}(P)$, where $\operatorname{Aut}(P)$ denotes the automorphism group of $P$.) It follows that there are 19 labelled interval orders or labelled semiorders on a 3-element set.

The following proposition collects some basic results on interval orders. We simply state them without proof. Only part (a) is needed in what follows (Lemma 5.6). We use the notation $i$ to denote an $i$-element chain and $P+Q$ to denote the disjoint union of the posets $P$ and $Q$.

Proposition 5.15. $\quad$ (a) A finite poset is an interval order if and only if it has no induced subposet isomorphic to $\mathbf{2}+\mathbf{2}$.

(b) A finite poset is a semiorder if and only if it has no induced subposet isomorphic to $\mathbf{2}+\mathbf{2}$ or $\mathbf{3}+\mathbf{1}$.

(c) A finite poset $P$ is a semiorder if and only if its elements can be ordered as $I_{1}, \ldots, I_{n}$ so that the incidence matrix of $P$ (i.e., the matrix $M=\left(m_{i j}\right)$, where $m_{i j}=1$ if $I_{i}<I_{j}$ and $m_{i j}=0$ otherwise) has the form shown below. Moreover, all such semiorders are nonisomorphic. 


$\left[\begin{array}{lll}0 & 0 & 0 \\ 0 & 0 & 0 \\ 0 & 0 & 0\end{array}\right] \quad\left[\begin{array}{lll}0 & 0 & 1 \\ 0 & 0 & 0 \\ 0 & 0 & 0\end{array}\right]\left[\begin{array}{lll}0 & 1 & 1 \\ 0 & 0 & 0 \\ 0 & 0 & 0\end{array}\right]\left[\begin{array}{lll}0 & 0 & 1 \\ 0 & 0 & 1 \\ 0 & 0 & 0\end{array}\right] \quad\left[\begin{array}{lll}0 & 1 & 1 \\ 0 & 0 & 1 \\ 0 & 0 & 0\end{array}\right]$

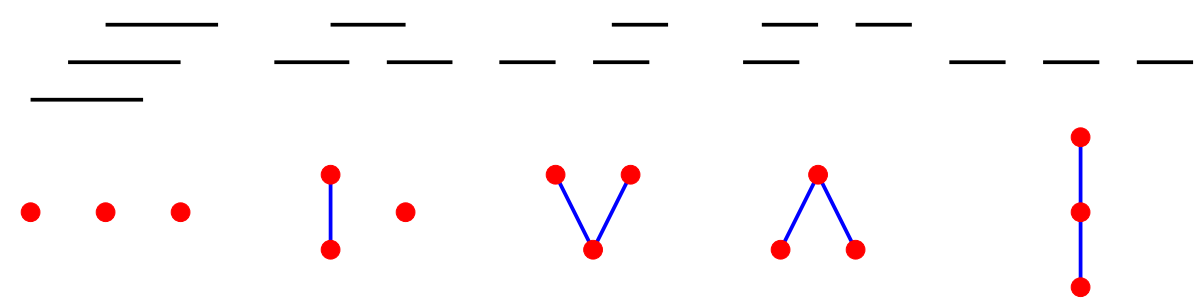

Figure 7. The semiorders with three elements

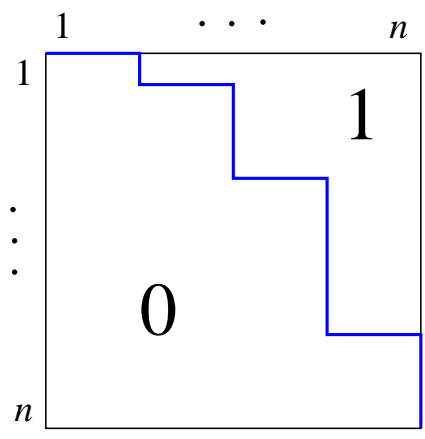

In (c) above, the southwest boundary of the positions of the 1's in $M$ form a lattice path which by suitable indexing goes from $(0,0)$ to $(n, n)$ with steps $(0,1)$ and $(1,0)$, never rising above $y=x$. Since the number of such lattice paths is the Catalan number $C_{n}$, it follows that the number of nonisomorphic $n$-element semiorders is $C_{n}$. Later (Proposition 5.17) we will give a proof based on properties of a certain arrangement. Figure 7 illustrates Proposition 5.15 (c) when $n=3$. It shows the matrices $M$, the corresponding set of unit intervals, and the associated semiorder.

Let $\ell_{1}, \ldots, \ell_{n}>0$ and set $\eta=\left(\ell_{1}, \ldots, \ell_{n}\right)$. Let $\mathcal{P}_{\eta}$ denote the set of all interval orders $P$ on $[n]$ such that there exist a set $I_{1}, \ldots, I_{n}$ of intervals corresponding to $P$ (with $I_{i}$ corresponding to $i \in P$ ) such that $\ell\left(I_{i}\right)=\ell_{i}$. In other words, $i \stackrel{P}{<} j$ if and only if $I_{i}$ lies entirely to the left of $I_{j}$. For instance, it follows from Figure 6 that $\# \mathcal{P}_{(1,1,1)}=19$.

We now come to the connection with arrangements. Given $\eta=\left(\ell_{1}, \ldots, \ell_{n}\right)$ as above, define the arrangement $\mathcal{J}_{\eta}$ in $\mathbb{R}^{n}$ by letting its hyperplanes be given by

$$
x_{i}-x_{j}=\ell_{i}, \quad i \neq j .
$$

(Note the condition $i \neq j$, not $i<j$.) Thus $\mathcal{J}_{\eta}$ has $\operatorname{rank} n-1$ and $n(n-1)$ hyperplanes (since $\ell_{i}>0$ ). Figure 8 shows the arrangement $\mathcal{J}_{(1,1,1)}$ in the space $\operatorname{ker}\left(x_{1}+x_{2}+x_{3}\right)$.

Proposition 5.16. Let $\eta \in \mathbb{R}_{+}^{n}$. Then $r\left(\mathcal{J}_{\eta}\right)=\# \mathcal{P}_{\eta}$. 


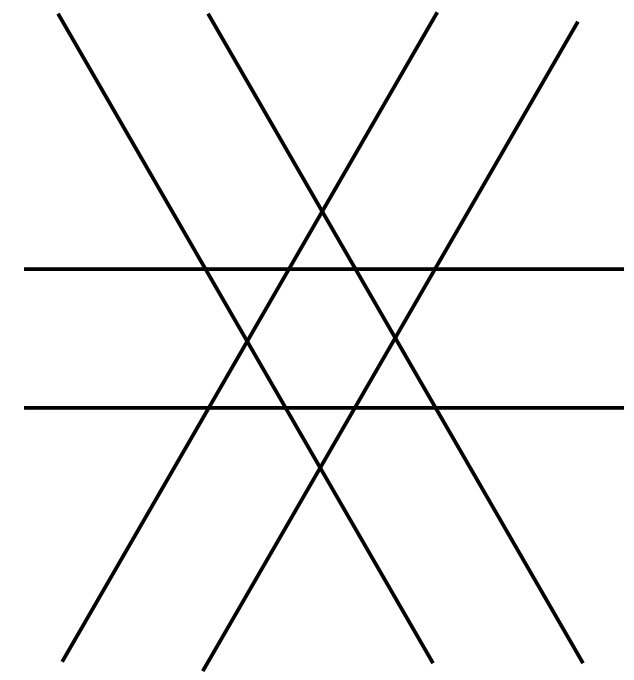

Figure 8. The arrangement $\mathcal{J}_{(1,1,1)}$ in the space $\operatorname{ker}\left(x_{1}+x_{2}+x_{3}\right)$

Proof. Let $\left(x_{1}, \ldots, x_{n}\right)$ belong to some region $R$ of $\mathcal{J}_{\eta}$. Define the interval $I_{i}=$ $\left[x_{i}-\ell_{i}, x_{i}\right]$. The region $R$ is determined by whether $x_{i}-x_{j}<\ell_{i}$ or $x_{i}-x_{j}>\ell_{i}$. Equivalently, $I_{i} \ngtr I_{j}$ or $I_{i}>I_{j}$ in the ordering on intervals that defines interval orders. Hence the number of possible interval orders corresponding to intervals $I_{1}, \ldots, I_{n}$ with $\ell\left(I_{i}\right)=\ell_{i}$ is just $r\left(\mathcal{J}_{\eta}\right)$.

Consider the case $\ell_{1}=\cdots=\ell_{n}=1$, so we are looking at the semiorder arrangement $x_{i}-x_{j}=1$ for $i \neq j$. We abbreviate $(1,1, \ldots, 1)$ as $1^{n}$ and denote this arrangement by $J_{1^{n}}$. By the proof of Proposition 5.16 the regions of $\mathcal{J}_{1^{n}}$ are in a natural bijection with semiorders on $[n]$.

Now note that $\mathcal{C}_{n}=\mathcal{J}_{1^{n}} \cup \mathcal{B}_{n}$, where $\mathcal{C}_{n}$ denotes the Catalan arrangement. Fix a region $R$ of $\mathcal{B}_{n}$, say $x_{1}<x_{2}<\cdots<x_{n}$. Then the number of regions of $\mathcal{J}_{1^{n}}$ that intersect $R$ is the number of semiorders on $[n]$ that correspond to (unit) intervals $I_{1}, \ldots, I_{n}$ with right endpoints $x_{1}<x_{2}<\cdots<x_{n}$. Another set $I_{1}^{\prime}, \ldots, I_{n}^{\prime}$ of unit intervals $I_{i}^{\prime}=\left[x_{i}^{\prime}-1, x_{i}^{\prime}\right]$ with $x_{1}^{\prime}<x_{2}^{\prime}<\cdots<x_{n}^{\prime}$ defines a different region from that defined by $I_{1}, \ldots, I_{n}$ if and only if the corresponding semiorders are nonisomorphic. It follows that the number of nonisomorphic semiorders on $[n]$ is equal to the number of regions of $\mathcal{J}_{1^{n}}$ intersecting the region $x_{1}<x_{2}<\cdots<x_{n}$ of $\mathcal{B}_{n}$. Since $\mathcal{C}_{n}=\mathcal{J}_{1^{n}} \cup \mathcal{B}_{n}$, there follows from Proposition 5.14 the following result of Wine and Freunde [38].

Proposition 5.17. The number $u(n)$ of nonisomorphic n-element semiorders is given by

$$
u(n)=\frac{1}{n !} r\left(\mathfrak{C}_{n}\right)=C_{n} .
$$

Figure 9 shows the nonisomorphic 3-element semiorders corresponding to the regions of $\mathcal{C}_{n}$ intersecting the region $x_{1}<x_{2}<\cdots<x_{n}$ of $\mathcal{B}_{n}$. on $[n]$.

We now come to the problem of determining $r\left(\mathcal{J}_{1^{n}}\right)$, the number of semiorders 


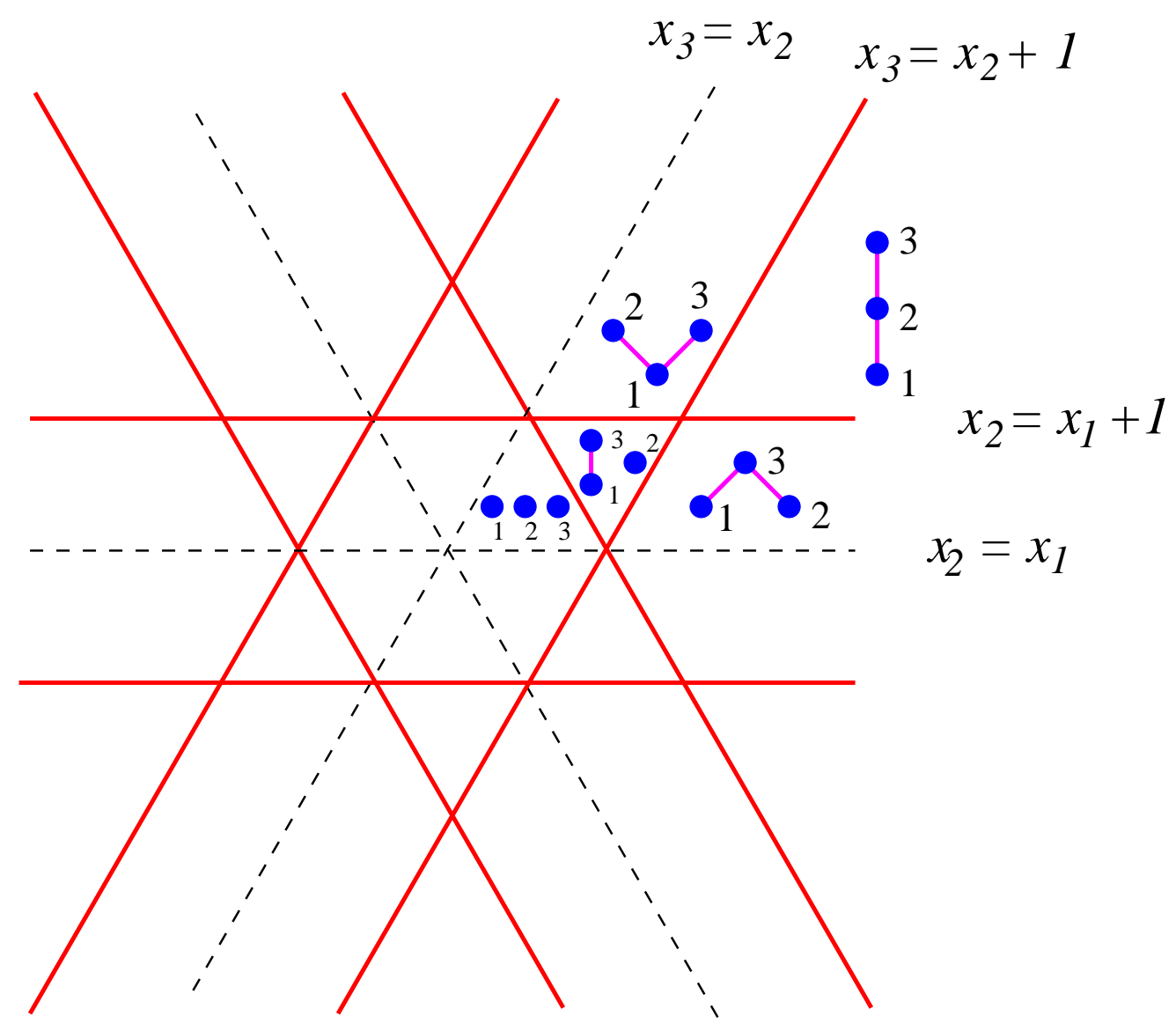

Figure 9. The nonisomorphic 3-element semiorders as regions of $\mathcal{C}_{1^{n}}$

Theorem 5.19. Fix distinct real numbers $a_{1}, a_{2}, \ldots, a_{m}>0$. Let $\mathcal{A}_{n}$ be the arrangement in $\mathbb{R}^{n}$ with hyperplanes

$$
\mathcal{A}_{n}: \quad x_{i}-x_{j}=a_{1}, \ldots, a_{m}, \quad i \neq j
$$

and let $\mathcal{A}_{n}^{*}=\mathcal{A}_{n} \cup \mathcal{B}_{n}$. Define

$$
\begin{aligned}
& F(x)=\sum_{n \geq 1} r\left(\mathcal{A}_{n}\right) \frac{x^{n}}{n !} \\
& G(x)=\sum_{n \geq 1} r\left(\mathcal{A}_{n}^{*}\right) \frac{x^{n}}{n !} .
\end{aligned}
$$

Then $F(x)=G\left(1-e^{-x}\right)$.

Proof. Let $c(n, k)$ denote the number of permutations $w$ of $n$ objects with $k$ cycles (in the disjoint cycle decomposition of $w$ ). The integer $c(n, k)$ is known as a signless Stirling number of the first kind and for fixed $k$ has the exponential generating 
function

$$
\sum_{n \geq 0} c(n, k) \frac{x^{n}}{n !}=\frac{1}{k !}\left(\log (1-x)^{-1}\right)^{k} .
$$

For futher information, see e.g. [31, pp. 17-20][32, (5.25)].

We have

$$
\begin{aligned}
F(x)=G\left(1-e^{-x}\right) \Leftrightarrow G(x) & =F\left(\log (1-x)^{-1}\right) \\
& =\sum_{k \geq 1} r\left(\mathcal{A}_{k}\right) \frac{1}{k !}\left(\log (1-x)^{-1}\right)^{k} \\
& =\sum_{k \geq 1} r\left(\mathcal{A}_{k}\right) \sum_{n \geq 0} c(n, k) \frac{x^{n}}{n !} .
\end{aligned}
$$

It follows that we need to show that

$$
r\left(\mathcal{A}_{n}^{*}\right)=\sum_{k=1}^{n} c(n, k) r\left(\mathcal{A}_{k}\right) .
$$

For simplicity we consider only the case $m=1$ and $a_{1}=1$, but the argument is completely analogous in the general case. When $m=1$ and $a_{1}=1$ we have that $r\left(\mathcal{A}_{n}^{*}\right)=n ! C_{n}$ and that $r\left(\mathcal{A}_{n}\right)$ is the number of semiorders on $[n]$. Thus it suffices to give a map $(P, w) \stackrel{\rho}{\mapsto} Q$, where $w \in \mathfrak{S}_{k}$ and $P$ is a semiorder whose elements are labelled by the cycles of $w$, and where $Q$ is an unlabelled $n$-element semiorder, such that $\rho$ is $n$ !-to- 1 , i.e., every $Q$ appears exactly $n$ ! times as an image of some $(P, w)$.

Choose $w \in \mathfrak{S}_{n}$ with $k$ cycles in $c(n, k)$ ways, and make these cycles the vertices of a semiorder $P$ in $r\left(\mathcal{A}_{k}\right)$ ways. Define a new poset $\rho(P, w)$ as follows: if the cycle $\left(c_{1}, \ldots, c_{j}\right)$ is an element of $P$, then replace it with an antichain with elements $c_{1}, \ldots, c_{j}$. Given $1 \leq c \leq n$, let $C(c)$ be the cycle of $w$ containing $c$. Define $c<d$ in $\rho(P, w)$ if $C(c)<C(d)$ in $P$. We illustrate this definition with $n=8$ and $w=(1,5,2)(3)(6,8)(4,7)$ :
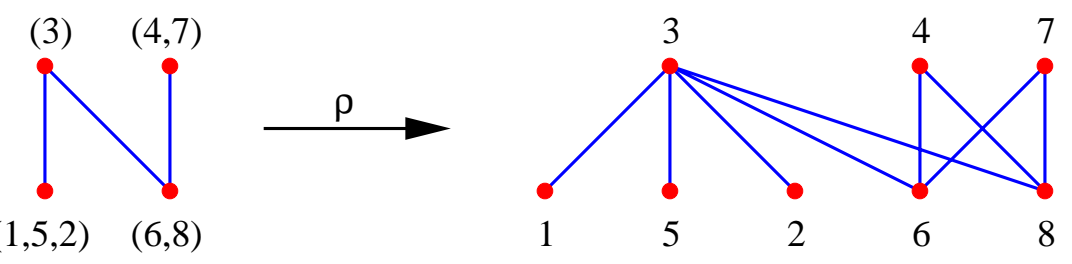

$$
(P, w) \quad Q=\rho(P, w)
$$

Given an unlabelled $n$-element semiorder $Q$, such as

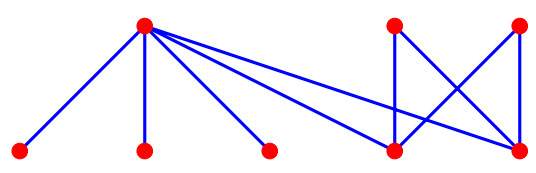


we now show that there are exactly $n$ ! pairs $(P, w)$ for which $\rho(P, w) \cong Q$. Call a pair of elements $x, y \in Q$ autonomous if for all $z \in Q$ we have

$$
x<z \Leftrightarrow y<z, \quad x>z \Leftrightarrow y>z .
$$

Equivalently, the map $\tau: Q \rightarrow Q$ transposing $x, y$ and fixing all other $z \in Q$ is an automorphism of $Q$. Clearly the relation of being autonomous is an equivalence relation. Partition $Q$ into its autonomous equivalence classes. Regard the elements of $Q$ as being distinguished, and choose a bijection (labeling) $\varphi: Q \rightarrow[n]$ (in $n$ ! ways). Fix a linear ordering (independent of $\varphi$ ) of the elements in each equivalence class. (The linear ordering of the elements in each equivalence class in the diagram below is left-to-right.)

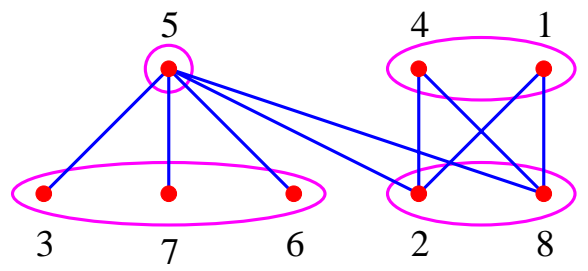

In each class, place a left parenthesis before each left-to-right maximum, and place a right parenthesis before each left parenthesis and at the end. (This is the bijection $\mathfrak{S}_{n} \rightarrow \mathfrak{S}_{n}, \hat{w} \mapsto w$, in $\left[\mathbf{3 1}\right.$, p. 17].) Merge the elements $c_{1}, c_{2}, \ldots, c_{j}$ (appearing in that order) between each pair of parentheses into a single element labelled with the cycle $\left(c_{1}, c_{2}, \ldots, c_{j}\right)$.

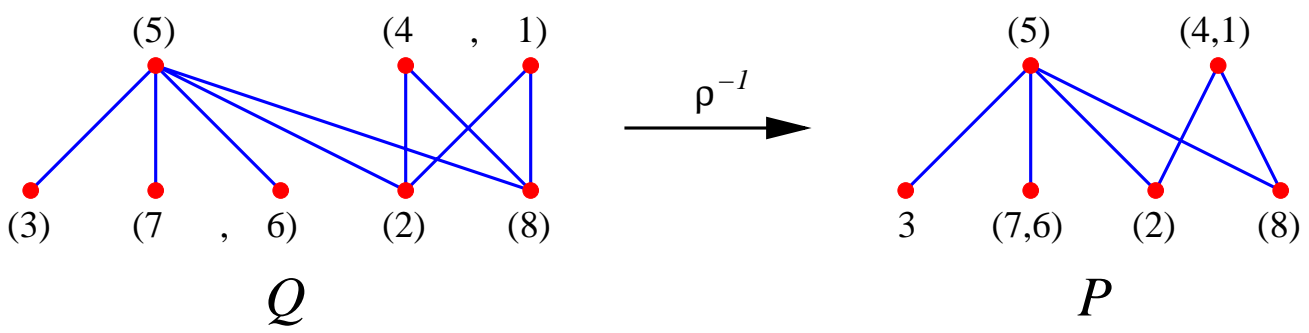

We have thus obtained a poset $P$ whose elements are labelled by the cycles of a permutation $w \in \mathfrak{S}_{n}$, such that $\rho(P, w)=Q$. For each unlabelled $Q$, there are exactly $n$ ! pairs $(P, w)$ (where the poset $P$ is labelled by the cycles of $w \in \mathfrak{S}_{n}$ ) for which $\rho(P, w) \cong Q$. Since by Proposition 5.17 there are $C_{n}$ nonisomorphic $n$-element semiorders, we get

$$
n ! C_{n}=\sum_{k=1}^{n} c(n, k) r\left(\mathcal{A}_{k}\right)
$$

Note. Theorem 5.19 can also be proved using Burnside's lemma (also called the Cauchy-Frobenius lemma) from group theory.

To test one's understanding of the proof of Theorem 5.19, consider why it doesn't work for all posets. In other words, let $f(n)$ denote the number of posets on $[n]$ and $g(n)$ the number of nonisomorphic $n$-element posets. Set $F(x)=\sum f(n) \frac{x^{n}}{n !}$ and $G(x)=\sum g(n) x^{n}$. Why doesn't the above argument show that $G(x)=F(1-$ 
$\left.e^{-x}\right)$ ? Let $Q=\mathbf{2}+\mathbf{2}$ (the unique obstruction to being an interval order, by Proposition 5.15(a)). The autonomous classes have one element each. Consider the two labelings $\varphi: Q \rightarrow[4]$ and the corresponding $\rho^{-1}$ :

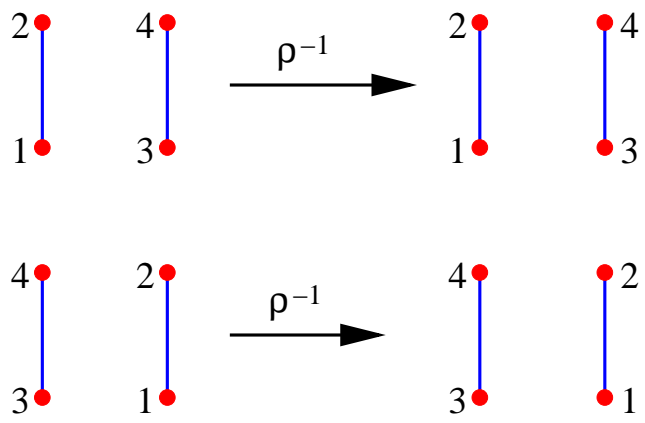

We obtain the same labelled posets in both cases, so the proof of Theorem 5.19 fails. The key property of interval orders that the proof of Theorem 5.19 uses implicitly is the following.

Lemma 5.6. If $\sigma: P \rightarrow P$ is an automorphism of the interval order $P$ and $\sigma(x)=\sigma(y)$, then $x$ and $y$ are autonomous.

Proof. Assume not. Then there exists an element $s \in P$ satisfying $s>x, s \ngtr y$ (or dually). Since $\sigma(x)=y$, there must exist $t \in P$ satisfying $t>y, t \ngtr x$. But then $\{x, s, y, t\}$ form an induced $\mathbf{2}+\mathbf{2}$, so by Proposition 5.15(a) $P$ is not an interval order.

Specializing $m=1$ and $a_{1}=1$ in Theorem 5.19 yields the following corollary, due first (in an equivalent form) to Chandon, Lemaire and Pouget [12].

Corollary 5.12. Let $f(n)$ denote the number of semiorders on $[n]$ (or n-element labelled semiorders). Then

$$
\sum_{n \geq 0} f(n) \frac{x^{n}}{n !}=C\left(1-e^{-x}\right),
$$

where

$$
C(x)=\sum_{n \geq 0} C_{n} x^{n}=\frac{1-\sqrt{1-4 x}}{2 x} .
$$

\subsection{Intervals with generic lengths}

A particularly interesting class of interval orders are those corresponding to intervals with specified generic lengths $\eta=\left(\ell_{1}, \ldots, \ell_{n}\right)$. Intuitively, this means that the intersection poset $P\left(\mathcal{J}_{\eta}\right)$ is as "large as possible." One way to make this precise is to say that $\eta$ is generic if $P\left(\mathcal{J}_{\eta}\right) \cong P\left(\mathcal{J}_{\eta^{\prime}}\right)$, where $\eta^{\prime}=\left(\ell_{1}^{\prime}, \ldots, \ell_{n}^{\prime}\right)$ and the $\ell_{i}^{\prime}$ 's are linearly independent over $\mathbb{Q}$. Thus if $\eta$ is generic, then the intersection poset $L\left(\mathcal{J}_{\eta}\right)$ does not depend on $\eta$, but rather only on $n$. In particular, $r\left(\mathcal{J}_{\eta}\right)$ does not depend on $\eta$ (always assuming $\eta$ is generic). Hence by Proposition 5.16, the number $\# \mathcal{P}_{\eta}$ of labelled interval orders corresponding to intervals $I_{1}, \ldots, I_{n}$ with $\ell\left(I_{i}\right)=\ell_{i}$ depends only on $n$. This fact is not at all obvious combinatorially, since the interval orders themselves do depend on $\eta$. For instance, it is easy to see that $\eta=(1,1.0001,1.001,1.01,1.1)$ is generic and that no corresponding interval order 
can be isomorphic to $\mathbf{4}+\mathbf{1}$. On the other hand, $\eta=(1,10,100,1000,10000)$ is also generic, but this time there is a corresponding interval order isomorphic to $\mathbf{4}+\mathbf{1}$. (See Exercise 17.)

The preceding discussion raises the question of computing $\# \mathcal{P}_{n}$ when $\eta$ is generic. We write $\mathcal{G}_{n}$ for the corresponding interval order $x_{i}-x_{j}=\ell_{i}, i \neq j$, since the intersection poset depends only on $n$. The following result is a nice application of arrangements to "pure" enumeration; no proof is known except the one sketched here.

Theorem 5.20. Let

$$
z=\sum_{n \geq 0} r\left(\mathcal{G}_{n}\right) \frac{x^{n}}{n !}=1+x+3 \frac{x^{2}}{2 !}+19 \frac{x^{3}}{3 !}+195 \frac{x^{4}}{4 !}+2831 \frac{x^{5}}{5 !}+\cdots .
$$

Define a power series

$$
y=1+x+5 \frac{x^{2}}{2 !}+46 \frac{x^{3}}{3 !}+631 \frac{x^{4}}{4}+\cdots
$$

by $1=y\left(2-e^{x y}\right)$. Equivalently,

$$
y=1+\left(\frac{1}{1+x} \log \frac{1+2 x}{1+x}\right)^{\langle-1\rangle} .
$$

Then $z$ is the unique power series satisfying $z^{\prime} / z=y^{2}, z(0)=1$.

Note. The condition $z^{\prime} / z=y^{2}$ can be rewritten as $z=\exp \int y^{2} d x$.

Sketch of proof. Putting $t=-1$ in Theorem 2.4 gives

$$
r\left(\mathcal{G}_{n}\right)=\sum_{\substack{\mathcal{B} \subseteq \mathcal{G}_{n} \\ \mathcal{B} \text { central }}}(-1)^{\# \mathcal{B}-\operatorname{rk}(\mathcal{B})} .
$$

Given a central subarrangement $\mathcal{B} \subseteq \mathcal{G}_{n}$, define a digraph (directed graph) $G_{\mathcal{B}}$ on $[n]$ by letting $i \rightarrow j$ be a (directed) edge if the hyperplane $x_{i}-x_{j}=\ell_{i}$ belongs to $\mathcal{B}$. One then shows that as an undirected graph $G_{\mathcal{B}}$ is bipartite, i.e., the vertices can be partitioned into two subsets $U$ and $V$ such that all edges connect a vertex in $U$ to a vertex in $V$. The pair $(U, V)$ is called a vertex bipartition of $G_{\mathcal{B}}$. Moreover, if $B$ is a block of $G_{\mathcal{B}}$ (as defined preceding Proposition 4.11), say with vertex bipartition $\left(U_{B}, V_{B}\right)$, then either all edges of $B$ are directed from $U_{B}$ to $V_{B}$, or all edges are directed from $V_{B}$ to $U_{B}$. It can also be seen that all such directed bipartite graphs can arise in this way. It follows that equation (46) can be rewritten

$$
r\left(\mathcal{G}_{n}\right)=(-1)^{n} \sum_{G}(-1)^{e(G)+c(G)} 2^{b(G)},
$$

where $G$ ranges over all (undirected) bipartite graphs on $[n], e(G)$ denotes the number of edges of $G$, and $b(G)$ denotes the number of blocks of $G$.

Equation (47) reduces the problem of determining $r(\mathcal{G})$ to a (rather difficult) problem in enumeration, whose solution may be found in $[\mathbf{2 5}, \S 6]$.

\subsection{Other examples}

There are two additional arrangements related to the braid arrangement that involve nice enumerative combinatorics. We merely repeat the definitions here from Lecture 1 and assemble some of their basic properties in Exercises 19-28. 
The Linial arrangement in $K^{n}$ is given by the hyperplanes $x_{i}-x_{j}=1,1 \leq i<$ $j \leq n$. It consists of "half" of the semiorder arrangement $\mathcal{J}_{1^{n}}$. Despite its similarity to $\mathcal{J}_{1^{n}}$, it is considerably more difficult to obtain its characteristic polynomial and other enumerative invariants. Finally the threshold arrangement in $K^{n}$ is given by the hyperplanes $x_{i}+x_{j}=0,1 \leq i<j \leq n$. It is a subarrangement of the Coxeter arrangements $\mathcal{A}\left(B_{n}\right)\left(=\mathcal{A}\left(C_{n}\right)\right)$ and $\mathcal{A}\left(\bar{D}_{n}\right)$. 


\section{Exercises}

(1) [2] Verify equation (37), viz.,

$$
\chi_{\mathcal{A}\left(D_{n}\right)}(t)=(t-1)(t-3) \cdots(t-(2 n-3)) \cdot(t-n+1) .
$$

(2) [2] Draw a picture of the projectivization of the Coxeter arrangement $\mathcal{A}\left(B_{3}\right)$, similar to Figure 1 of Lecture 1.

(3) (a) [2] An embroidered permutation of $[n]$ consists of a permutation $w$ of $[n]$ together with a collection $\mathcal{E}$ of ordered pairs $(i, j)$ such that:

- $1 \leq i<j \leq n$ for all $(i, j) \in \mathcal{E}$.

- If $(i, j)$ and $(h, k)$ are distinct elements of $\mathcal{E}$, then it is false that $i \leq h \leq k \leq j$.

- If $(i, j) \in \mathcal{E}$ then $w(i)<w(j)$.

For instance, the three embroidered permutations $(w, \mathcal{E})$ of $[2]$ are given by $(12, \emptyset),(12,\{(1,2)\})$, and $(21, \emptyset)$. Give a bijective proof that the number $r\left(\mathcal{S}_{n}\right)$ of regions of the Shi arrangement $\mathcal{S}_{n}$ is equal to the number of embroidered permutations of $[n]$.

(b) $[2+]$ A parking function of length $n$ is a sequence $\left(a_{1}, \ldots, a_{n}\right) \in \mathbb{P}^{n}$ whose increasing rearrangement $b_{1} \leq b_{2} \leq \cdots \leq b_{n}$ satisfies $b_{i} \leq i$. For instance, the parking functions of length three are 11, 12, 21. Give a bijective proof that the number of parking functions of length $n$ is equal to the number of embroidered permutations of $[n]$.

(c) [3-] Give a combinatorial proof that the number of parking functions of length $n$ is equal to $(n+1)^{n-1}$.

(4) $[2+]$ Show that if $\mathcal{S}_{n}$ denotes the Shi arrangement, then the cone $c \mathcal{S}_{n}$ is not supersolvable for $n \geq 3$.

(5) [2] Show that if $f: \mathbb{P} \rightarrow R$ and $h: \mathbb{N} \rightarrow R$ are related by equation (40) (with $h(0)=1)$, then equation (39) holds.

(6) (a) [2] Compute the characteristic polynomial of the arrangement $\mathcal{B}_{n}^{\prime}$ in $\mathbb{R}^{n}$ with defining polynomial

$$
Q(x)=\left(x_{1}-x_{n}-1\right) \prod_{1 \leq i<j \leq n}\left(x_{i}-x_{j}\right) .
$$

In other words, $\mathcal{B}_{n}^{\prime}$ consists of the braid arrangement together with the hyperplane $x_{1}-x_{n}=1$.

(b) [5-] Is $c \mathcal{B}_{n}^{\prime}$ (the cone over $\mathcal{B}_{n}^{\prime}$ ) supersolvable?

(7) $[2+]$ Let $1 \leq k \leq n$. Find the characteristic polynomial of the arrangement $S_{n, k}$ in $\mathbb{R}^{n}$ defined by

$$
\begin{array}{ll}
x_{i}-x_{j}=0 & \text { for } \quad 1 \leq i<j \leq n \\
x_{i}-x_{j}=1 & \text { for } \quad 1 \leq i<j \leq k .
\end{array}
$$

(8) $[2+]$ Let $1 \leq k \leq n$. Find the characteristic polynomial of the arrangement $\mathcal{C}_{n, k}$ in $\mathbb{R}^{n}$ defined by

$$
\begin{aligned}
x_{i}=0 & \text { for } \quad 1 \leq i \leq n \\
x_{i} \pm x_{j}=0 & \text { for } \quad 1 \leq i<j \leq n \\
x_{i}+x_{j}=1 & \text { for } \quad 1 \leq i<j \leq k .
\end{aligned} .
$$

In particular, show that $r\left(\mathcal{C}_{n, k}\right)=2^{n-k} n !\left(\begin{array}{c}2 k \\ k\end{array}\right)$. 
(9) (a) $[2+]$ Let $\mathcal{A}_{n}$ be the arrangement in $\mathbb{R}^{n}$ with hyperplanes $x_{i}=0$ for all $i$, $x_{i}=x_{j}$ for all $i<j$, and $x_{i}=2 x_{j}$ for all $i \neq j$. Show that

$$
\chi_{\mathcal{A}_{n}}(t)=(t-1)(t-n-2)_{n-1},
$$

where $(x)_{m}=x(x-1) \cdots(x-m+1)$. In particular, $r\left(\mathcal{A}_{n}\right)=2(2 n+$ $1) ! /(n+2)$ !. Can this be seen combinatorially? (This last question has not been worked on.)

(b) $[2+]$ Now let $\mathcal{A}_{n}$ be the arrangement in $\mathbb{R}^{n}$ with hyperplanes $x_{i}=x_{j}$ for all $i<j$ and $x_{i}=2 x_{j}$ for all $i \neq j$. Show that

$$
\chi_{\mathcal{A}_{n}}(t)=(t-1)(t-n-2)_{n-3}\left(t^{2}-(3 n-1) t+3 n(n-1)\right) .
$$

In particular, $r\left(\mathcal{A}_{n}\right)=6 n^{2}(2 n-1) ! /(n+2)$ !. Again, a combinatorial proof can be asked for.

(c) [5-] Modify. For instance, what about the arrangement with hyperplanes $x_{i}=0$ for all $i, x_{i}=x_{j}$ for all $i<j$, and $x_{i}=2 x_{j}$ for all $i<j$ ? (This example is actually not difficult.) Or $x_{i}=0$ for all $i, x_{i}=x_{j}$ for all $i<j$, $x_{i}=2 x_{j}$ for all $i \neq j$, and $x_{i}=3 x_{j}$ for all $i \neq j$ ?

(10) (a) $[2+]$ For $n \geq 1$ let $\mathcal{A}_{n}$ be an arrangement in $\mathbb{R}^{n}$ such that every $H \in \mathcal{A}_{n}$ is parallel to a hyperplane of the form $x_{i}=c x_{j}$, where $c \in \mathbb{R}$. Just as in the definition of an exponential sequence of arrangements, define for every subset $S$ of $[n]$ the arrangement

$$
\mathcal{A}_{n}^{S}=\left\{H \in \mathcal{A}_{n}: H \text { is parallel to some } x_{i}=c x_{j} \text {, where } i, j \in S\right\} .
$$

Suppose that for every such $S$ we have $L_{\mathcal{A}_{n}^{S}} \cong L_{\mathcal{A}_{k}}$, where $k=\# S$. Let

$$
\begin{aligned}
F(x) & =\sum_{n \geq 0}(-1)^{n} r\left(\mathcal{A}_{n}\right) \frac{x^{n}}{n !} \\
G(x) & =\sum_{n \geq 0}(-1)^{\operatorname{rank}\left(\mathcal{A}_{n}\right)} b\left(\mathcal{A}_{n}\right) \frac{x^{n}}{n !} .
\end{aligned}
$$

Show that

$$
\sum_{n \geq 0} \chi_{\mathcal{A}_{n}}(t) \frac{x^{n}}{n !}=\frac{G(x)^{(t+1) / 2}}{F(x)^{(t-1) / 2}}
$$

Verify that this formula is correct for the braid arrangement.

(b) [2] Simplify equation (48) when each $\mathcal{A}_{n}, n \geq 1$, is a central arrangement. Make sure that your simplification is valid for the coordinate hyperplane arrangement.

(11) $[2+]$ Let $\mathcal{R}_{0}\left(\mathcal{C}_{n}\right)$ denote the set of regions of the Catalan arrangement $\mathcal{C}_{n}$ contained in the regions $x_{1}>x_{2}>\cdots>x_{n}$ of $\mathcal{B}_{n}$. Let $\hat{R}$ be the unique region in $\mathcal{R}_{0}\left(\mathcal{C}_{n}\right)$ whose closure contains the origin. For $R \in \mathcal{R}_{0}\left(\mathcal{C}_{n}\right)$, let $X_{R}$ be the set of hyperplanes $H \in \mathcal{C}_{n}$ such that $\hat{R}$ and $R$ lie on different sides of $H$. Let $W_{n}=\left\{X_{R}: R \in \mathcal{R}_{0}\left(\mathcal{C}_{n}\right)\right\}$, ordered by inclusion. 

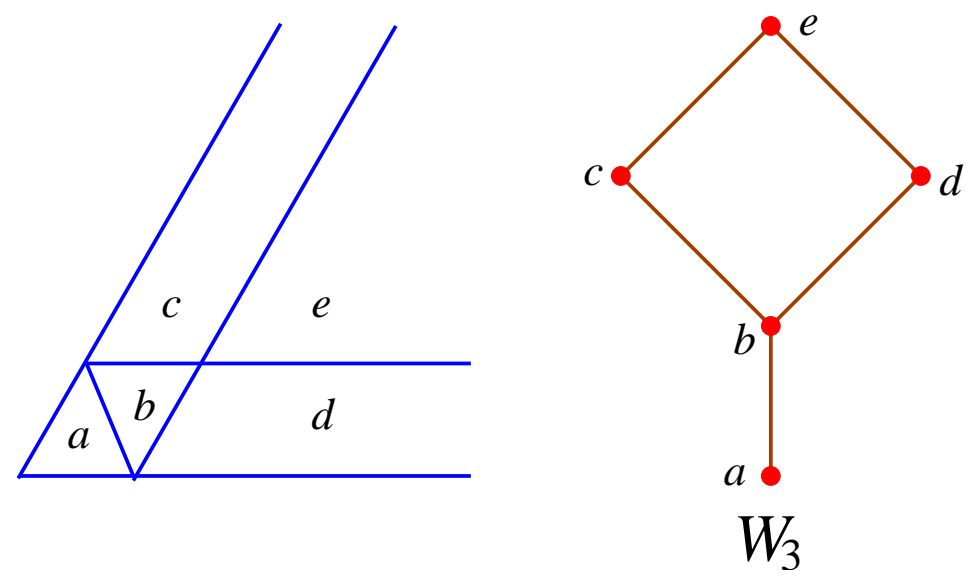

Let $P_{n}$ be the poset of intervals $[i, j], 1 \leq i<j \leq n$, ordered by reverse inclusion.
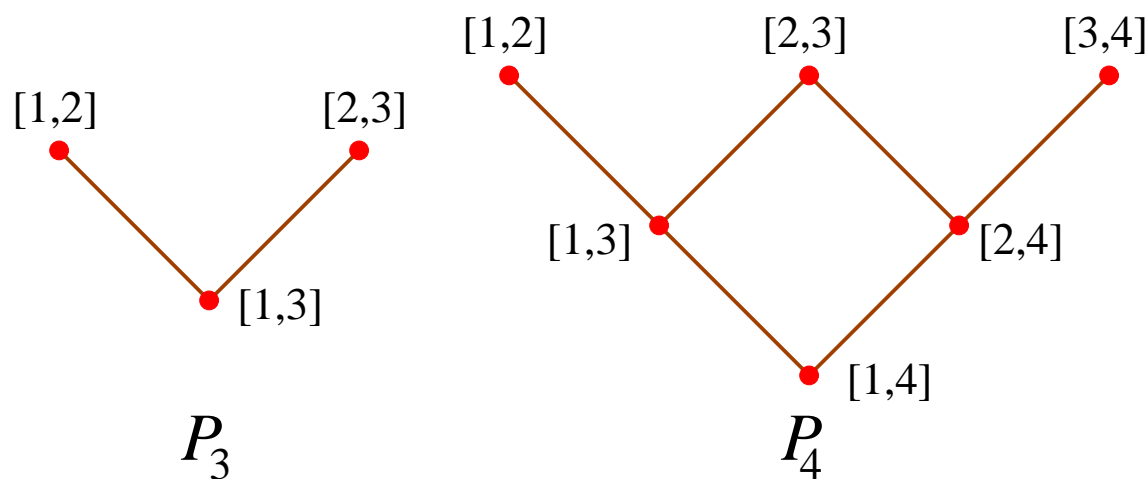

Show that $W_{n} \cong J\left(P_{n}\right)$, the lattice of order ideals of $P_{n}$. (An order ideal of a poset $P$ is a subset $I \subseteq P$ such that if $x \in I$ and $y \leq x$, then $y \in I$. Define $J(P)$ to be the set of order ideals of $P$, ordered by inclusion. See [31, Thm. 3.4.1].)

(12) [2] Use the finite field method to prove that

$$
\chi \mathrm{e}_{n}(t)=t(t-n-1)(t-n-2)(t-n-3) \cdots(t-2 n+1),
$$

where $\mathcal{C}_{n}$ denotes the Catalan arrangement.

(13) $[2+]$ Let $k \in \mathbb{P}$. Find the number of regions and characteristic polynomial of the extended Catalan arrangement

$$
\mathcal{C}_{n}(k): x_{i}-x_{j}=0, \pm 1, \pm 2, \ldots, \pm k, \text { for } 1 \leq i<j \leq n .
$$

Generalize Exercise 11 to the arrangements $\mathcal{C}_{n}(k)$.

(14) [3-] Let $\mathcal{S}_{n}^{B}$ denote the arrangement

$$
\begin{aligned}
x_{i} \pm x_{j} & =0,1, & & 1 \leq i<j \leq n \\
2 x_{i} & =0,1, & & 1 \leq i \leq n,
\end{aligned}
$$

called the Shi arrangement of type B. Find the characteristic polynomial and number of regions of $\mathcal{S}_{n}^{B}$. Is there a "nice" bijective proof of the formula for the number of regions? 
(15) [5-] Let $1 \leq k \leq n$. Find the number of regions (or more generally the characteristic polynomial) of the arrangement (in $\mathbb{R}^{n}$ )

$$
x_{i}-x_{j}= \begin{cases}1, & 1 \leq i \leq k \\ 2, & k+1 \leq i \leq n,\end{cases}
$$

for all $i \neq j$. Thus we are counting interval orders on $[n]$ where the elements $1,2, \ldots, k$ correspond to intervals of length one, while $k+1, \ldots, n$ correspond to intervals of length two. Is it possible to count such interval orders up to isomorphism (i.e., the unlabelled case)? What if the length 2 is replaced instead by a generic length $a$ ?

(16) $[2+]$ A double semiorder on $[n]$ consists of two binary relations $<$ and $\ll$ on $[n]$ that arise from a set $x_{1}, \ldots, x_{n}$ of real numbers as follows:

$$
\begin{array}{lll}
i<j & \text { if } & x_{i}<x_{j}-1 \\
i \ll j & \text { if } & x_{i}<x_{j}-2 .
\end{array}
$$

If we associate the interval $I_{i}=\left[x_{i}-2, x_{i}\right]$ with the point $x_{i}$, then we are specifying whether $I_{i}$ lies to the left of the midpoint of $I_{j}$, entirely to the left of $I_{j}$, or neither. It should be clear what is meant for two double semiorders to be isomorphic.

(a) [2] Draw interval diagrams of the 12 nonisomorphic double semiorders on $\{1,2,3\}$.

(b) [2] Let $\rho_{2}(n)$ denote the number of double semiorders on $[n]$. Find an arrangement $\mathcal{J}_{n}^{(2)}$ satisfying $r\left(\mathcal{J}_{n}^{(2)}\right)=\rho_{2}(n)$.

(c) $[2+]$ Show that the number of nonisomorphic double semiorders on $[n]$ is given by $\frac{1}{2 n+1}\left(\begin{array}{c}3 n \\ n\end{array}\right)$.

(d) [2-] Let $F(x)=\sum_{n \geq 0} \frac{1}{2 n+1}\left(\begin{array}{c}3 n \\ n\end{array}\right) x^{n}$. Show that

$$
\sum_{n \geq 0} \rho_{2}(n) \frac{x^{n}}{n !}=F\left(1-e^{-x}\right) .
$$

(e) [2] Generalize to " $k$-semiorders," where ordinary semiorders (or unit interval orders) correspond to $k=1$ and double semiorders to $k=2$.

(17) $[1+]$ Show that intervals of lengths 1,1.0001,1.001,1.01, 1.1 cannot form an interval order isomorphic to $\mathbf{4 + 1}$, but that such an interval order can be formed if the lengths are $1,10,100,1000,10000$.

(18) [5-] What more can be said about interval orders with generic interval lengths? For instance, consider the two cases: (a) interval lengths very near each other (e.g., 1, 1.001, 1.01, 1.1), and (b) interval lengths superincreasing (e.g., 1, 10, 100, 1000). Are there finitely many obstructions to being such an interval order? Can the number of unlabelled interval orders of each type be determined? (Perhaps the numbers are the same, but this seems unlikely.)

(19) (a) [3] Let $\mathcal{L}_{n}$ denote the Linial arrangement, say in $\mathbb{R}^{n}$. Show that

$$
\chi_{\mathcal{L}_{n}}(t)=\frac{t}{2^{n}} \sum_{k=1}^{n}\left(\begin{array}{l}
n \\
k
\end{array}\right)(t-k)^{n-1} .
$$

(b) $[1+]$ Deduce from (a) that

$$
\frac{\chi_{\mathcal{L}_{n}}(t)}{t}=\frac{(-1)^{n} \chi_{\mathcal{L}_{n}}(-t+n)}{-t+n} .
$$



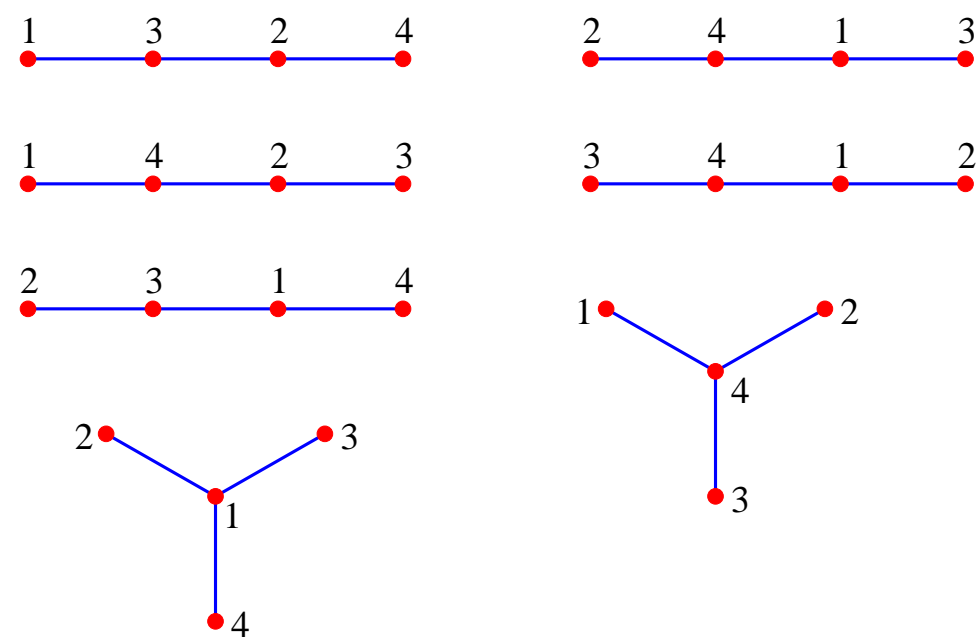

Figure 10. The seven alternating trees on the vertex set [4]

(20) (a) [3-] An alternating tree on the vertex set $[n]$ is a tree on $[n]$ such that every vertex is either less than all its neighbors or greater than all its neighbors. Figure 10 shows the seven alternating trees on [4]. Deduce from Exercise $19(\mathrm{a})$ that $r\left(\mathcal{L}_{n}\right)$ is equal to the number of alternating trees on $[n+1]$.

(b) [5] Find a bijective proof of (a), i.e., give an explicit bijection between the regions of $\mathcal{L}_{n}$ and the alternating trees on $[n+1]$.

(21) [3-] Let

$$
\chi_{\mathcal{L}_{n}}(t)=a_{n} t^{n}-a_{n-1} t^{n-1}+\cdots+(-1)^{n-1} a_{1} t .
$$

Deduce from Exercise 19(a) that $a_{i}$ is the number of alternating trees on the vertex set $0,1, \ldots, n$ such that vertex 0 has degree (number of adjacent vertices) $i$.

(22) (a) $[2+]$ Let $P(t) \in \mathbb{C}[t]$ have the property that every (complex) zero of $P(t)$ has real part $a$. Let $z \in \mathbb{C}$ satisfy $|z|=1$. Show that every zero of the polynomial $P(t-1)+z P(t)$ has real part $a+\frac{1}{2}$.

(b) $[2+]$ Deduce from (a) and Exercise 19(a) that every zero of the polynomial $\chi_{\mathcal{L}_{n}}(t) / t$ has real part $n / 2$. This result is known as the "Riemann hypothesis for the Linial arrangement."

(23) (a) [2-] Compute $\lim _{n \rightarrow \infty} b\left(\mathcal{S}_{n}\right) / r\left(\mathcal{S}_{n}\right)$, where $\mathcal{S}_{n}$ denotes the Shi arrangement. (b) [3] Do the same for the Linial arrangement $\mathcal{L}_{n}$.

(24) $[2+]$ Let $\mathcal{L}_{n}$ denote the Linial arrangement in $\mathbb{R}^{n}$. Fix an integer $r \neq 0, \pm 1$, and let $\mathcal{M}_{n}(r)$ be the arrangement in $\mathbb{R}^{n}$ defined by $x_{i}=r x_{j}, 1 \leq i<j \leq n$, together with the coordinate hyperplanes $x_{i}=0$. Find a relationship between $\chi_{\mathcal{L}_{n}}(t)$ and $\chi_{\mathcal{M N}_{n}(r)}(t)$ without explicitly computing these characteristic polynomials.

(25) (a) [3-] A threshold graph on $[n]$ may be defined recursively as follows: (i) the empty graph $\emptyset$ is a threshold graph, (ii) if $G$ is a threshold graph, then so is the disjoint union of $G$ and a single vertex, and (iii) if $G$ is a threshold graph, then so is the graph obtained by adding a new vertex $v$ and connecting it to every vertex of $G$. Let $\mathcal{T}_{n}$ denote the threshold arrangement. Show that $r\left(\mathcal{T}_{n}\right)$ is the number of threshold graphs on $[n]$. 
(b) $[2+]$ Deduce from (a) that

$$
\sum_{n \geq 0} r\left(\mathcal{T}_{n}\right) \frac{x^{n}}{n !}=\frac{e^{x}(1-x)}{2-e^{x}} .
$$

(c) $[1+]$ Deduce from Exercise 10 that

$$
\sum_{n \geq 0} \chi_{\mathcal{T}_{n}}(t) \frac{x^{n}}{n !}=(1+x)\left(2 e^{x}-1\right)^{(t-1) / 2} .
$$

(26) [5-] Let

$$
\chi_{\mathcal{T}_{n}}(t)=t^{n}-a_{n-1} t^{n-1}+\cdots+(-1)^{n} a_{0} .
$$

For instance,

$$
\begin{aligned}
& \chi_{\mathcal{T}_{3}}(t)=t^{3}-3 t^{2}+3 t-1 \\
& \chi_{\mathcal{T}_{4}}(t)=t^{4}-6 t^{3}+15 t^{2}-17 t+7 \\
& \chi_{\mathcal{T}_{5}}(t)=t^{5}-10 t^{4}+45 t^{3}-105 t^{2}+120 t-51 .
\end{aligned}
$$

By Exercise 25(a), $a_{0}+a_{1}+\cdots+a_{n-1}+1$ is the number of threshold graphs on the vertex set $[n]$. Give a combinatorial interpretation of the numbers $a_{i}$ as the number of threshold graphs with a certain property.

(27) (a) $[1+]$ Find the number of regions of the "Linial threshold arrangement"

$$
x_{i}+x_{j}=1, \quad 1 \leq i<j \leq n .
$$

(b) [5-] Find the number of regions, or even the characteristic polynomial, of the "Shi threshold arrangement"

$$
x_{i}+x_{j}=0,1, \quad 1 \leq i<j \leq n .
$$

(28) [3-] Let $\mathcal{A}_{n}$ denote the "generic threshold arrangement" (in $\mathbb{R}^{n}$ ) $x_{i}+x_{j}=a_{i j}$, $1 \leq i<j \leq n$, where the $a_{i j}$ 's are generic. Let

$$
T(x)=\sum_{n \geq 1} n^{n-2} \frac{x^{n}}{n !}
$$

the generating function for labelled trees on $n$ vertices. Let

$$
R(x)=\sum_{n \geq 1} n^{n-1} \frac{x^{n}}{n !}
$$

the generating function for rooted labelled trees on $n$ vertices. Show that

$$
\begin{aligned}
\sum_{n \geq 0} r\left(\mathcal{A}_{n}\right) \frac{x^{n}}{n !} & =e^{T(x)-\frac{1}{2} R(x)}\left(\frac{1+R(x)}{1-R(x)}\right)^{1 / 4} \\
& =1+x+2 \frac{x^{2}}{2 !}+8 \frac{x^{3}}{3 !}+54 \frac{x^{4}}{4 !}+533 \frac{x^{5}}{5 !}+6934 \frac{x^{6}}{6 !}+\cdots
\end{aligned}
$$

(29) $[2+]$ Fix $n \geq 1$. Let $f(k, n, r)$ be the number of $k \times n(0,1)$-matrices $A$ over the rationals such that all rows of $A$ are distinct, every row has at least one 1 , and $\operatorname{rank}(A)=r$. Let $g_{n}(q)$ be the number of $n$-tuples $\left(a_{1}, \ldots, a_{n}\right) \in \mathbb{F}_{q}^{n}$ such that no nonempty subset of the entries sums to $0\left(\right.$ in $\mathbb{F}_{q}$ ). Show that for $p \gg 0$, where $q=p^{d}$, we have

$$
g_{n}(q)=\sum_{k, r} \frac{(-1)^{k}}{k !} f(k, n, r) q^{n-r} .
$$


(The case $k=0$ is included, corresponding to the empty matrix, which has rank 0$. 



\section{LECTURE 6 Separating Hyperplanes}

\subsection{The distance enumerator}

Let $\mathcal{A}$ be a real arrangement, and let $R$ and $R^{\prime}$ be regions of $\mathcal{A}$. A hyperplane $H \in \mathcal{A}$ separates $R$ and $R^{\prime}$ if $R$ and $R^{\prime}$ lie on opposite sides of $H$. In this chapter we will consider some results dealing with separating hyperplanes. To begin, let

$$
\operatorname{sep}\left(R, R^{\prime}\right)=\left\{H \in \mathcal{A}: H \text { separates } R \text { and } R^{\prime}\right\} \text {. }
$$

Define the distance $d\left(R, R^{\prime}\right)$ between the regions $R$ and $R^{\prime}$ to be the number of hyperplanes $H \in \mathcal{A}$ that separate $R$ and $R^{\prime}$, i.e.,

$$
d\left(R, R^{\prime}\right)=\# \operatorname{sep}\left(R, R^{\prime}\right) .
$$

It is easily seen that $d$ is a metric on the $\operatorname{set} \mathcal{R}(\mathcal{A})$ of regions of $\mathcal{A}$, i.e.,

- $d\left(R, R^{\prime}\right) \geq 0$ for all $R, R^{\prime} \in \mathcal{R}(\mathcal{A})$, with equality if and only if $R=R^{\prime}$

- $d\left(R, R^{\prime}\right)=d\left(R^{\prime}, R\right)$ for all $R, R^{\prime} \in \mathcal{R}(\mathcal{A})$

- $d\left(R, R^{\prime}\right)+d\left(R^{\prime}, R^{\prime \prime}\right) \geq d\left(R, R^{\prime \prime}\right)$ for all $R, R^{\prime}, R^{\prime \prime} \in \mathcal{R}(\mathcal{A})$.

Now fix a region $R_{0} \in \mathcal{R}(\mathcal{A})$, called the base region. The distance enumerator of $\mathcal{A}$ (with respect to $R_{0}$ ) is the polynomial

$$
D_{\mathcal{A}, R_{0}}(t)=\sum_{R \in \mathcal{R}(\mathcal{A})} t^{d\left(R_{0}, R\right)} .
$$

We simply write $D_{\mathcal{A}}(t)$ if no confusion will result. Also define the weak order (with respect to $R_{0}$ ) of $\mathcal{A}$ to be the partial order $W_{\mathcal{A}}$ on $\mathcal{R}(\mathcal{A})$ given by

$$
R \leq R^{\prime} \text { if } \operatorname{sep}\left(R_{0}, R\right) \subseteq \operatorname{sep}\left(R_{0}, R^{\prime}\right) .
$$

It is easy to see that $W_{\mathcal{A}}$ is a partial ordering of $\mathcal{R}(\mathcal{A})$. The poset $W_{\mathcal{A}}$ is graded by distance from $R_{0}$, i.e., $R_{0}$ is the $\hat{0}$ element of $\mathcal{R}(\mathcal{A})$, and all saturated chains between $R_{0}$ and $R$ have length $d\left(R_{0}, R\right)$.

Figure 1 shows three arrangements in $\mathbb{R}^{2}$, with $R_{0}$ labelled 0 and then each $R \neq R_{0}$ labelled $d\left(R_{0}, R\right)$. Under each arrangement is shown the corresponding weak order $W_{\mathcal{A}}$. The first arrangement is the braid arrangement $\mathcal{B}_{3}$ (essentialized). Here the choice of base region does not affect the distance enumerator $1+2 t+2 t^{2}+$ $t^{3}=(1+t)\left(1+t+t^{2}\right)$ nor the weak order. On the other hand, the second two arrangements of Figure 1 are identical, but the choice of $R_{0}$ leads to different weak orders and different distance enumerators, viz., $1+2 t+2 t^{2}+t^{3}$ and $1+3 t+2 t^{2}$. 

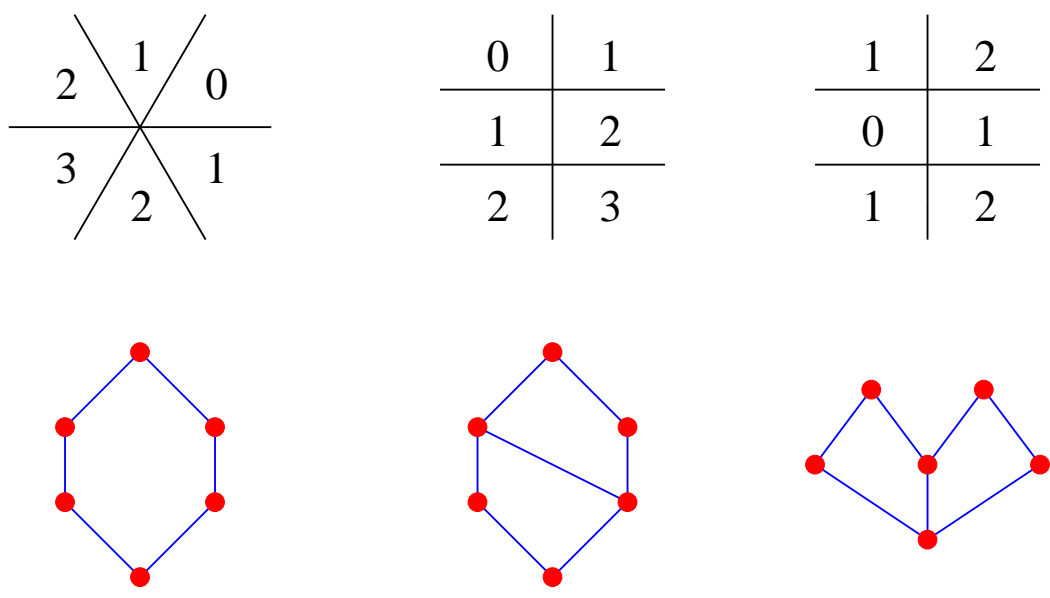

Figure 1. Examples of weak orders

Consider now the braid arrangement $\mathcal{B}_{n}$. We know from Example 1.3 that the regions of $\mathcal{B}_{n}$ are in one-to-one correspondence with the permutations of $[n]$, viz.,

$$
\begin{aligned}
\mathcal{R}\left(\mathcal{B}_{n}\right) & \leftrightarrow \mathfrak{S}_{n} \\
x_{w(1)}>x_{w(2)}>\cdots>x_{w(n)} & \leftrightarrow w .
\end{aligned}
$$

Given $w=a_{1} a_{2} \cdots a_{n} \in \mathfrak{S}_{n}$, define an inversion of $w$ to be a pair $(i, j)$ such that $i<j$ and $a_{i}>a_{j}$. Let $\ell(w)$ denote the number of inversions of $w$. The inversion sequence $\operatorname{IS}(w)$ of $w$ is the vector $\left(c_{1}, \cdots, c_{n}\right)$, where

$$
c_{j}=\#\left\{i: i<j, w^{-1}(j)<w^{-1}(i)\right\} .
$$

Note that the condition $w^{-1}(j)<w^{-1}(i)$ is equivalent to $i$ appearing to the right of $j$ in $w$. For instance, $\operatorname{IS}(461352)=(0,0,1,3,1,4)$. The inversion sequence is a modified form of the inversion table or of the code of $w$, as defined in the literature, e.g., [31, p. 21] [32, solution to Exer. 6.19(x)]. For our purposes the inversion sequence is the most convenient. It is clear from the definition of $\operatorname{IS}(w)$ that if $\operatorname{IS}(w)=\left(c_{1}, \ldots, c_{n}\right)$ then $\ell(w)=c_{1}+\cdots+c_{n}$. Moreover, is easy to see (Exercise 2$)$ that a sequence $\left(c_{1}, \ldots, c_{n}\right) \in \mathbb{N}^{n}$ is the inversion sequence of a permutation $w \in \mathfrak{S}_{n}$ if and only if $c_{i} \leq i-1$ for $1 \leq i \leq n$. It follows that

$$
\begin{aligned}
\sum_{w \in \mathfrak{S}_{n}} t^{\ell(w)} & =\sum_{\substack{\left(c_{1}, \ldots, c_{n}\right) \\
0 \leq c_{i} \leq i-1}} t^{c_{1}+\cdots+c_{n}} \\
& =\left(\sum_{c_{1}=0}^{0} t^{c_{1}}\right) \cdots\left(\sum_{c_{n}=0}^{n-1} t^{c_{n}}\right) \\
& =1 \cdot(1+t)\left(1+t+t^{2}\right) \cdots\left(1+t+\cdots+t^{n-1}\right),
\end{aligned}
$$

a standard result on permutation statistics [31, Cor. 1.3.10].

Denote by $R_{w}$ the region of $\mathcal{B}_{n}$ corresponding to $w \in \mathfrak{S}_{n}$, and choose $R_{0}=R_{\text {id }}$, where $\mathrm{id}=12 \cdots n$, the identity permutation. Suppose that $R_{u}, R_{v} \in \mathcal{R}\left(\mathcal{B}_{n}\right)$ such that $\operatorname{sep}\left(R_{0}, R_{v}\right)=\{H\} \cup \operatorname{sep}\left(R_{0}, u\right)$ for some $H \in \mathcal{B}_{n}, H \notin \operatorname{sep}\left(R_{0}, R_{u}\right)$. Thus $R_{u}$ and $R_{v}$ are separated by a single hyperplane $H$, and $R_{0}$ and $R_{u}$ lie on the same side of $H$. Suppose that $H$ is given by $x_{i}=x_{j}$ with $i<j$. Then $i$ and $j$ appear 


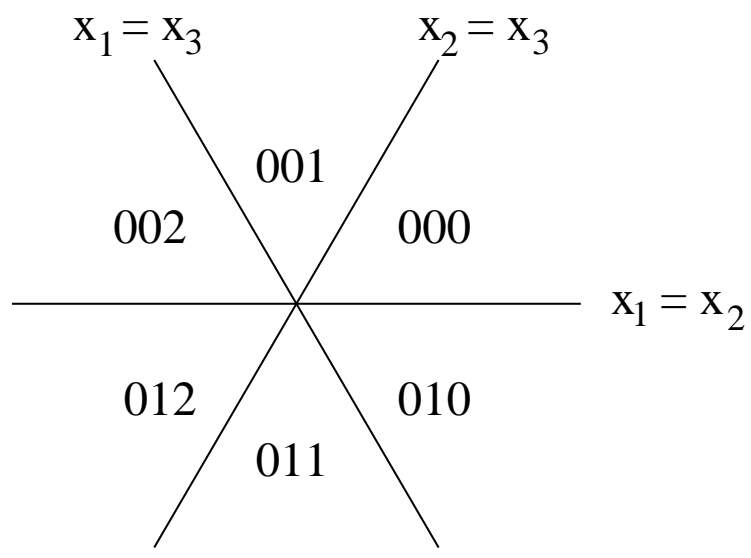

Figure 2. The inversion sequence labeling of the regions of $\mathcal{B}_{3}$

consecutively in $u$ written as a word $a_{1} \cdots a_{n}$ (since $H$ is a bounding hyperplane of the region $R_{u}$ ) and $i$ appears to the left of $j$ (since $R_{0}$ and $R_{u}$ lie on the same side of $H$ ). Thus $v$ is obtained from $u$ by transposing the adjacent pair $i j$ of letters. It follows that $\ell(v)=\ell(u)+1$. If $u(k)=i$ and we let $s_{k}=(k, k+1)$, the adjacent transposition interchanging $k$ and $k+1$, then $v=u s_{k}$.

The following result is an immediate consequence of equation (49) and mathematical induction.

Proposition 6.18. Let $R_{0}=R_{\mathrm{id}}$ as above. If $w \in \mathfrak{S}_{n}$ then $d\left(R_{0}, R_{w}\right)=\ell(w)$. Moreover,

$$
D_{\mathcal{B}_{n}}(t)=(1+t)\left(1+t+t^{2}\right) \cdots\left(1+t+\cdots+t^{n-1}\right) .
$$

There is a somewhat different approach to Proposition 6.18 which will be generalized to the Shi arrangement. We label each region $R$ of $\mathcal{B}_{n}$ recursively by a vector $\lambda(R)=\left(c_{1}, \ldots, c_{n}\right) \in \mathbb{N}^{n}$ as follows.

- $\lambda\left(R_{0}\right)=(0,0, \ldots, 0)$

- Let $e_{i}$ denote the $i$ th unit coordinate vector in $\mathbb{R}^{n}$. If the regions $R$ and $R^{\prime}$ of $\mathcal{B}_{n}$ are separated by the single hyperplane $H$ with the equation $x_{i}=x_{j}$, $i<j$, and if $R$ and $R_{0}$ lie on the same side of $H$, then $\lambda\left(R^{\prime}\right)=\lambda(R)+e_{j}$.

Figure 2 shows the labels $\lambda(R)$ for $\mathcal{B}_{3}$.

Proposition 6.19. Let $w \in \mathfrak{S}_{n}$. Then $\lambda\left(R_{w}\right)=\operatorname{IS}(w)$, the inversion sequence of $w$.

Proof. The proof is a straightforward induction on $\ell(w)$. If $\ell(w)=0$, then $w=\mathrm{id}$ and

$$
\lambda\left(R_{\mathrm{id}}\right)=\lambda\left(R_{0}\right)=(0,0, \ldots, 0)=\mathrm{IS}(\mathrm{id}) .
$$

Suppose $w=a_{1} \cdots a_{n}$ and $\ell(w)>0$. For some $1 \leq k \leq n-1$ we must have $a_{k}=j>i=a_{k+1}$. Thus $\ell\left(w s_{k}\right)=\ell(w)-1$. Hence by induction we may assume $\lambda\left(w s_{k}\right)=\operatorname{IS}\left(w s_{k}\right)$. The hyperplane $x_{i}=x_{j}$ separates $R_{w}$ from $R_{w s_{k}}$. Hence by the definition of $\lambda$ we have

$$
\lambda\left(R_{w}\right)=\lambda\left(R_{w s_{k}}\right)+e_{j}=\operatorname{IS}\left(w s_{k}\right)+e_{j}
$$


By the definition of the inversion sequence we have $\operatorname{IS}\left(w s_{k}\right)+e_{j}=\operatorname{IS}(w)$, and the proof follows.

NotE. The weak order $W_{\mathcal{B}_{n}}$ of the braid arrangement is an interesting poset, usually called the weak order or weak Bruhat order on $\mathfrak{S}_{n}$. For instance $[\mathbf{1 4}][\mathbf{1 7}][\mathbf{3 0}]$, the number of maximal chains of $W_{\mathcal{B}_{n}}$ is given by

$$
\frac{\left(\begin{array}{l}
n \\
2
\end{array}\right) !}{1^{n-1} 3^{n-2} 5^{n-3} \cdots(2 n-3)}
$$

For additional properties of $W_{\mathcal{B}_{n}}$, see [5].

\subsection{Parking functions and tree inversions}

Some beautiful enumerative combinatorics is associated with the distance enumerator of the Shi arrangement $\mathcal{S}_{n}$ (for a suitable choice of $R_{0}$ ). The fundamental combinatorial object needed for this purpose is a parking function.

Definition 6.15. Let $n \in \mathbb{P}$. A parking function of length $n$ is a sequence $\left(a_{1}, \ldots, a_{n}\right) \in \mathbb{Z}^{n}$ whose increasing rearrangment $b_{1} \leq b_{2} \leq \cdots \leq b_{n}$ satisfies $1 \leq b_{i} \leq i$ for $1 \leq i \leq n$. Equivalently, the sequence $\left(b_{1}-1, \ldots, b_{n}-1\right)$ is the inversion sequence of some permutation $w \in \mathfrak{S}_{n}$.

The parking functions of length at most 3 are given as follows:

\begin{tabular}{lllllllll} 
& & 1 & \multicolumn{9}{c}{11} & 12 & 21 \\
111 & 112 & 121 & 211 & 113 & 131 & 311 & 122 \\
212 & 221 & 123 & 132 & 213 & 231 & 312 & 321
\end{tabular}.

The term "parking function" $[\mathbf{2 1}, \S 6]$ arises from the following scenario. A oneway street has parking spaces labelled $1,2, \ldots, n$ in that order. There are $n$ cars $C_{1}, \ldots, C_{n}$ which enter the street one at a time and try to park. Each car $C_{i}$ has a preferred space $a_{i} \in[n]$. When it is $C_{i}$ 's turn to look for a space, it immediately drives to space $a_{i}$ and then parks in the first available space. For instance, if $\left(a_{1}, a_{2}, a_{3}, a_{4}\right)=(2,1,2,3)$, then $C_{1}$ parks in space 2 , then $C_{2}$ parks in space 1 , then $C_{3}$ goes to space 2 (which is occupied) parks in space 3 (the next available), and finally $C_{4}$ goes to space 3 and parks in space 4 . On the other hand, if $\left(a_{1}, a_{2}, a_{3}, a_{4}\right)=(3,1,4,3)$, then $C_{4}$ is unable to park, since its preferred space 3 and all subsequent spaces are already occupied. It is not hard to show (Exercise 3 ) that all the cars can park if and only if $\left(a_{1}, \ldots, a_{n}\right)$ is a parking function.

A basic question concerning parking functions (to be refined in Theorem 6.22) is their enumeration. The next result was first proved by Konheim and Weiss [21, $\S 6]$; we give an elegant proof due to Pollak (described in [26][16, p. 13]).

Proposition 6.20. The number of parking functions of length $n$ is $(n+1)^{n-1}$.

Proof. Arrange $n+1$ (rather than $n$ ) parking spaces in a circle, labelled $1, \ldots, n+1$ in counterclockwise order. We still have $n$ cars $C_{1}, \ldots, C_{n}$ with preferred spaces $\left(a_{1}, \ldots, a_{n}\right)$, but now we can have $1 \leq a_{i} \leq n+1$ (rather than $1 \leq a_{i} \leq n$ ). Each car enters the circle one at a time at their preferred space and then drives counterclockwise until encountering an empty space, in which case the car parks there. Note the following:

- All the cars can always park, since they drive in a circle and will always find an empty space.

- After all cars have parked there will be one empty space. 


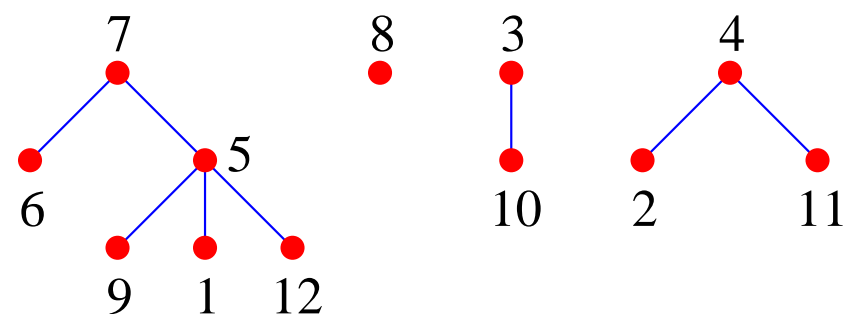

Figure 3. A rooted forest on [12]

- The sequence $\left(a_{1}, \ldots, a_{n}\right)$ is a parking function if and only if the empty space after all the cars have parked is $n+1$.

- If the preference sequence $\left(a_{1}, \ldots, a_{n}\right)$ produces the empty space $i$ at the end, then the sequence $\left(a_{1}+k, \ldots, a_{n}+k\right)$ (taking entries modulo $n+1$ so they always lie in the set $[n+1]$ ) produces the empty space $i+k$ (modulo $n+1)$.

It follows that exactly one of the sequences $\left(a_{1}+k, \ldots, a_{n}+k\right)$ (modulo $\left.n+1\right)$, where $1 \leq k \leq n+1$, is a parking function. There are $(n+1)^{n}$ sequences $\left(a_{1}, \ldots, a_{n}\right)$ in all, so exactly $(n+1)^{n} /(n+1)=(n+1)^{n-1}$ are parking functions.

Many readers will have recognized that the number $(n+1)^{n-1}$ is closely related to the enumeration of trees. Indeed, there is an intimate connection between trees and parking functions. We therefore now present some background material on trees. A tree on $[n]$ is a connected graph without cycles on the vertex set $[n]$. A rooted tree is a pair $(T, i)$, where $T$ is a tree and $i$ is a vertex of $T$, called the root. We draw trees in the standard computer science manner with the root at the top and all edges emanating downwards. A forest on $[n]$ is a graph $F$ on the vertex set $[n]$ for which every (connected) component is a tree. Equivalently, $F$ has no cycles. A rooted forest (also called a planted forest) is a forest for which every component has a root, i.e., for each tree $T$ of the forest select a vertex $i_{T}$ of $T$ to be the root of T. A standard result in enumerative combinatorics (e.g., [32, Prop. 5.3.2]) states that the number of rooted forests on $[n]$ is $(n+1)^{n-1}$.

An inversion of a rooted forest $F$ on $[n]$ is a pair $(i, j)$ of vertices such that $i<j$ and $j$ appears on the (unique) path from $i$ to the root of the tree in which $i$ occurs. Write $\operatorname{inv}(F)$ for the number of inversions of $F$. For instance, the rooted forest $F$ of Figure 3 has the inversions $(6,7),(1,7),(5,7),(1,5)$, and $(2,4)$, so $\operatorname{inv}(F)=5$.

Define the inversion enumerator $I_{n}(t)$ of rooted forests on $[n]$ by

$$
I_{n}(t)=\sum_{F} t^{\operatorname{inv}(F)}
$$

where $F$ ranges over all rooted forests on $[n]$. Figure 4 shows the 16 rooted forests on [3] with their number of inversions written underneath, from which it follows that

$$
I_{3}(t)=6+6 t+3 t^{2}+t^{3} .
$$

We collect below the three main results on $I_{n}(t)$. They are theorems in "pure" enumeration and have no direct connection with arrangements. The first result, due to Mallows and Riordan [23], gives a remarkable connection with connected graphs. 


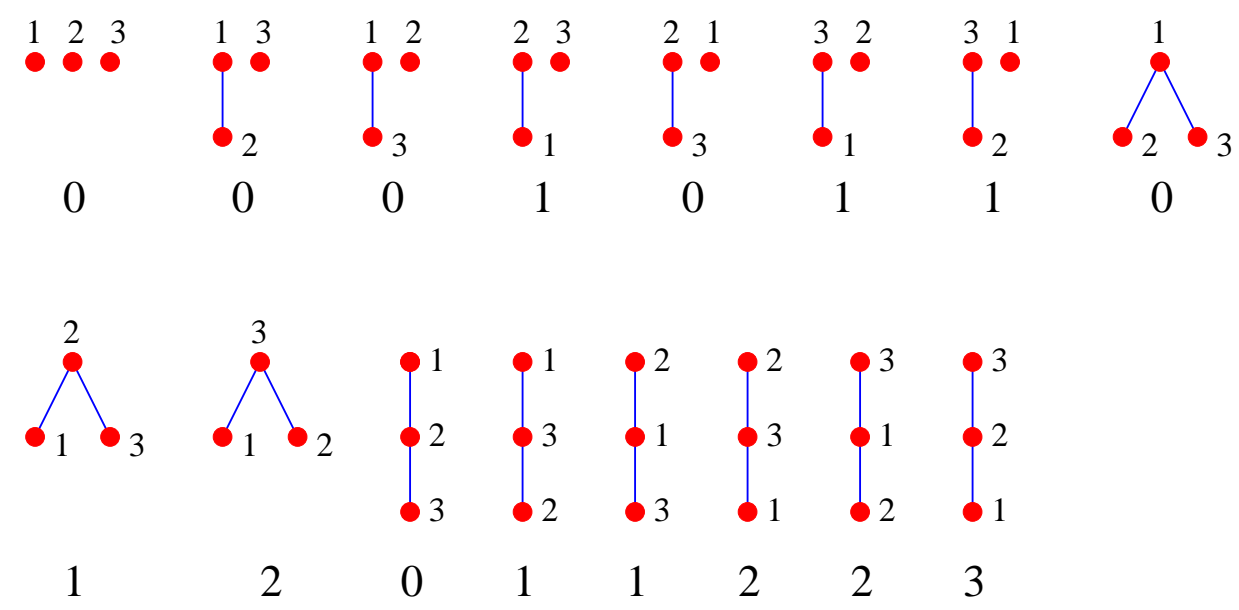

Figure 4. The 16 rooted forests on [3] and their number of inversions

Theorem 6.21. We have

$$
I_{n}(1+t)=\sum_{G} t^{e(G)-n},
$$

where $G$ ranges over all connected (simple) graphs on the vertex set $[0, n]=\{0,1, \ldots, n\}$ and $e(G)$ denotes the number of edges of $G$.

For instance,

$$
I_{3}(1+t)=16+15 t+6 t^{2}+t^{3} .
$$

Thus, for instance, there are 15 connected graphs on [0,3] with four edges. Three of these are 4-cycles and twelve consist of a triangle with an incident edge. The enumeration of connected graphs is well-understood [32, Exam. 5.2.1]. In particular, if

$$
C_{n}(t)=\sum_{G} t^{e(G)},
$$

where $G$ ranges over all connected (simple) graphs on $[n]$, then

$$
\sum_{n \geq 0} C_{n}(t) \frac{x^{n}}{n !}=\log \sum_{n \geq 1}(1+t)^{\left(\begin{array}{c}
n \\
2
\end{array}\right)} \frac{x^{n}}{n !}
$$

Thus Theorem 6.21 "determines" $I_{n}(t)$. There is an alternative way to state this result that doesn't involve the logarithm function.

Corollary 6.13. We have

$$
\sum_{n \geq 0} I_{n}(t)(t-1)^{n} \frac{x^{n}}{n !}=\frac{\sum_{n \geq 0} t^{\left(\begin{array}{c}
n+1 \\
2
\end{array}\right)} \frac{x^{n}}{n !}}{\sum_{n \geq 0} t_{\left(\begin{array}{c}
n \\
2
\end{array}\right)} \frac{x^{n}}{n !}}
$$

The third result, due to Kreweras [22], connects inversion enumerators with parking functions. Let $\mathrm{PF}_{n}$ denote the set of parking function of length $n$. 
Theorem 6.22. Let $n \geq 1$. Then

$$
t^{\left(\begin{array}{c}
n \\
2
\end{array}\right)} I_{n}(1 / t)=\sum_{\left(a_{1}, \ldots, a_{n}\right) \in \mathrm{PF}_{n}} t^{a_{1}+\cdots+a_{n}-n} .
$$

We now give proofs of Theorem 6.21, Corollary 6.13, and Theorem 6.22.

Proof of Theorem 6.21 (sketch). The following elegant proof is due to Gessel and Wang [18]. Let $G$ be a connected graph on $[0, n]$. Start at vertex 0 and let $T$ be the "depth-first spanning tree," i.e., move to the largest unvisited neighbor or else (if there is no unvisited neighbor) backtrack. The edges traversed when all vertices are visited are the edges of the spanning tree $T$. Remove the vertex 0 and root the trees that remain at the neighbors of 0 . Denote this rooted forest by $F_{G}$.
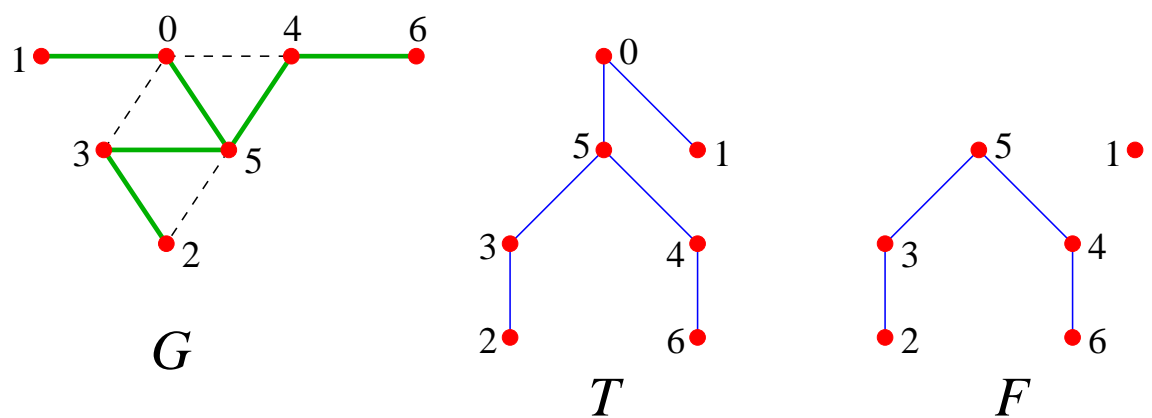

Given a spanning forest $F$ on $[n]$, what connected graphs $G$ on $[0, n]$ satisfy $F=F_{G}$ ? The answer, whose straightforward verification we leave to the reader, is the following. Add the vertex 0 to $F$ and connect it the roots of $F$, obtaining $T$. Clearly $G$ consists of $T$ with some added edges $i j$. The edge $i j$ can be added to $T$ if and only if the path from 0 to $j$ contains $i$ (or vice versa), and if $i^{\prime}$ is the next vertex after $i$ on the path from $i$ to $j$, then $\left(j, i^{\prime}\right)$ is an inversion of $F$. Thus each inversion of $F$ corresponds to a possible edge that can be added to $T$, and these edges can be added or not added independently. It follows that

$$
\begin{aligned}
\sum_{G: F=F_{G}} t^{e(G)} & =t^{e(T)}(1+t)^{\operatorname{inv}(F)} \\
& =t^{n}(1+t)^{\operatorname{inv}(F)} .
\end{aligned}
$$

Summing on all rooted forests $F$ on $[n]$ gives

$$
\begin{aligned}
\sum_{G} t^{e(G)} & =t^{n} \sum_{F}(1+t)^{\operatorname{inv}(F)} \\
& =t^{n} I_{n}(1+t),
\end{aligned}
$$

where $G$ ranges over all connected graphs on $[0, n]$.

Proof of Corollary 6.13. By equation (50) and Theorem 6.21 we have

$$
\sum_{n \geq 0} t^{n-1} I_{n-1}(1+t) \frac{x^{n}}{n !}=\log \sum_{n \geq 0}(1+t)^{\left(\begin{array}{c}
n \\
2
\end{array}\right)} \frac{x^{n}}{n !} .
$$

Substituting $t-1$ for $t$ gives

$$
\sum_{n \geq 0}(t-1)^{n-1} I_{n-1}(t) \frac{x^{n}}{n !}=\log \sum_{n \geq 0} t_{\left(\begin{array}{c}
n \\
2
\end{array}\right)} \frac{x^{n}}{n !} .
$$

Now differentiate both sides with respect to $x$ to obtain equation (51). 
Proof of Theorem 6.22. Let

$$
\begin{aligned}
J_{n}(t) & =\sum_{\left(a_{1}, \ldots, a_{n}\right) \in \mathrm{PF}_{n}} t^{\left(\begin{array}{c}
n \\
2
\end{array}\right)-\sum\left(a_{i}-1\right)} \\
& =\sum_{\left(a_{1}, \ldots, a_{n}\right) \in \mathrm{PF}_{n}} t^{\left(\begin{array}{c}
n+1 \\
2
\end{array}\right)-\sum a_{i}} .
\end{aligned}
$$

Claim \#1:

$$
J_{n+1}(t)=\sum_{i=0}^{n}\left(\begin{array}{l}
n \\
i
\end{array}\right)\left(1+t+t^{2}+\cdots+t^{i}\right) J_{i}(t) J_{n-i}(t) .
$$

Proof of claim. Choose $0 \leq i \leq n$, and let $S$ be an $i$-element subset of $[n]$. Choose also $\alpha \in \mathrm{PF}_{i}, \beta \in \mathrm{PF}_{n-i}$, and $0 \leq j \leq i$. Form a vector $\gamma=\left(\gamma_{1}, \ldots, \gamma_{n+1}\right)$ by placing $\alpha$ at the positions indexed by $S$, placing $\left(\beta_{1}+i+1, \ldots, \beta_{n-i}+i+1\right)$ at the positions indexed by $[n]-S$, and placing $j+1$ at position $n+1$. For instance, suppose $n=7, i=3, S=\{2,3,6\}, \alpha=(1,2,1), \beta=(2,1,4,2)$, and $j=1$. Then $\gamma=(6,1,2,5,8,1,6,2) \in \mathrm{PF}_{8}$. It is easy to check that in general $\gamma \in \mathrm{PF}_{n+1}$. Note that

$$
\sum_{k=1}^{n+1} \gamma_{k}=\sum_{k=1}^{i} \alpha_{k}+\sum_{k=1}^{n-i} \beta_{k}+(n-i)(i+1)+j+1,
$$

so

$$
\left(\begin{array}{c}
n+2 \\
2
\end{array}\right)-\sum \gamma_{k}=\left(\begin{array}{c}
i+1 \\
2
\end{array}\right)-\sum \alpha_{k}+\left(\begin{array}{c}
n-i+1 \\
2
\end{array}\right)-\sum \beta_{k}+i-j .
$$

Equation (53) then follows if the map $(i, S, \alpha, \beta, j) \mapsto \gamma$ is a bijection, i.e., given $\gamma \in \mathrm{PF}_{n+1}$, we can uniquely obtain $(i, S, \alpha, \beta, j)$ so that $(i, S, \alpha, \beta, j) \mapsto \gamma$. Now given $\gamma$, note that $i+1$ is the largest number that can replace $\gamma_{n+1}$ so that we still have a parking function. Once $i$ is determined, the rest of the argument is clear, proving the claim.

Note. Several bijections are known between the set of all rooted forests $F$ on $[n]$ (or rooted trees on $[0, n]$ ) and the set $\mathrm{PF}_{n}$ of all parking functions $\left(a_{1}, \ldots, a_{n}\right)$ of length $n$, but none of them have the property that $\operatorname{inv}(F)=a_{1}+\cdots+a_{n}-n$. Hence a direct bijective proof of Theorem 6.22 is not known. It would be interesting to find such a proof (Exercise 4).

Claim \#2:

$$
I_{n+1}(t)=\sum_{i=0}^{n}\left(\begin{array}{c}
n \\
i
\end{array}\right)\left(1+t+t^{2}+\cdots+t^{i}\right) I_{i}(t) I_{n-i}(t) .
$$

Proof of claim. We give a proof due to G. Kreweras [22]. Let $F$ be a rooted forest on $S \subseteq[n], \# S=i$, and let $G$ be a rooted forest on $\bar{S}=[n]-S$. Let $u_{1}<\cdots<u_{i}$ be the vertices of $F$, and set $u_{i+1}=n+1$. Choose $1 \leq j \leq i+1$. For all $m \geq j$ replace $u_{m}$ by $u_{m+1}$. (If $j=i+1$, then do nothing.) This gives a labelled forest $F^{\prime}$ on $(S \cup\{n+1\})-\left\{u_{j}\right\}$. Let $T^{\prime}$ be the labelled tree obtained from $F^{\prime}$ by adjoining the root $u_{j}$ and connecting it to the roots of $F^{\prime}$. Keep $G$ the same. We obtain a rooted forest $H$ on $[n+1]$ satisfying

$$
\operatorname{inv}(H)=j-1+\operatorname{inv}(F)+\operatorname{inv}(G) .
$$




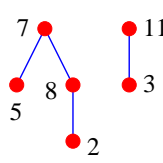

F

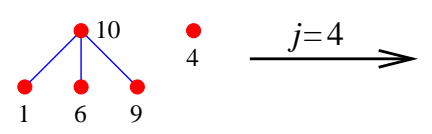

$G$

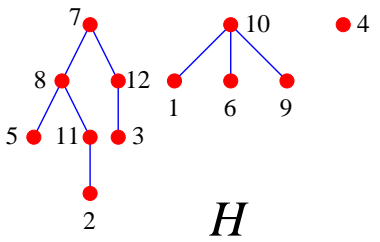

This process gives a bijection $(S, F, G, j) \mapsto H$, where $S \subseteq[n], F$ is a rooted forest on $S, G$ is a rooted forest on $\bar{S}, 1 \leq j \leq 1+\# S$, and $H$ is a rooted forest on $[n+1]$. Hence

$$
\sum_{i=0}^{n} \sum_{\substack{S \subseteq[n] \\ \# S=i}} I_{i}(t) I_{n-i}(t)\left(1+t+\cdots+t^{i}\right)=I_{n+1}(t),
$$

and the claim follows.

The initial conditions $I_{0}(t)=J_{0}(t)=1$ agree, so by the two claims we have $I_{n}(t)=J_{n}(t)$ for all $n \geq 0$. The proof of equation (52) follows by substituting $1 / t$ for $t$.

\subsection{The distance enumerator of the Shi arrangement}

Recall that the Shi arrangement $\mathcal{S}_{n}$ is given by the defining polynomial

$$
Q_{S_{n}}=\prod_{1 \leq i<j \leq n}\left(x_{i}-x_{j}\right)\left(x_{i}-x_{j}-1\right) .
$$

Let $K=\mathbb{R}$, and let $R_{0}$ denote the region

$$
x_{1}>x_{2}>\cdots>x_{n}>x_{1}-1,
$$

so $x \in R_{0}$ if and only if $0 \leq x_{i}-x_{j} \leq 1$ for all $i<j$. We define a labeling $\lambda: \mathcal{R}\left(\mathcal{S}_{n}\right) \rightarrow \mathbb{N}^{n}$ of the regions of $\mathcal{S}_{n}$ as follows.

- $\lambda\left(R_{0}\right)=(0,0, \ldots, 0)$

- If the regions $R$ and $R^{\prime}$ of $\mathcal{S}_{n}$ are separated by the single hyperplane $H$ with the equation $x_{i}=x_{j}, i<j$, and if $R$ and $R_{0}$ lie on the same side of $H$, then $\lambda\left(R^{\prime}\right)=\lambda(R)+e_{j}$ (exactly as for the braid arrangement).

- If the regions $R$ and $R^{\prime}$ of $\mathcal{B}_{n}$ are separated by the single hyperplane $H$ with the equation $x_{i}=x_{j}+1, i<j$, and if $R$ and $R_{0}$ lie on the same side of $H$, then $\lambda\left(R^{\prime}\right)=\lambda(R)+e_{i}$.

Note that the labeling $\lambda$ is well-defined, since $\lambda(R)$ depends only on $\operatorname{sep}\left(R_{0}, R\right)$. Figure 5 shows the labeling $\lambda$ for the case $n=3$.

Theorem 6.23. All labels $\lambda(R), R \in \mathcal{R}\left(\mathcal{S}_{n}\right)$, are distinct, and

$$
\mathrm{PF}_{n}=\left\{\left(a_{1}+1, \ldots, a_{n}+1\right):\left(a_{1}, \ldots, a_{n}\right)=\lambda(R) \text { for some } R \in \mathcal{R}\left(\mathcal{S}_{n}\right)\right\} .
$$

In other words, the labels $\lambda(R)$ for $R \in \mathcal{R}\left(\mathcal{S}_{n}\right)$ are obtained from the labels $\lambda(R)$ for $R \in \mathcal{R}\left(\mathcal{B}_{n}\right)$ by permuting coordinates in all possible ways. This remarkable fact seems much more difficult to prove than the corresponding result for $\mathcal{B}_{n}$, viz., the labels $\lambda(R)$ for $\mathcal{B}_{n}$ consist of the sequences $\left(a_{1}, \ldots, a_{n}\right)$ with $0 \leq a_{i} \leq i-1$ (an immediate consequence of Proposition 6.19 and Exercise 2.

Proof of Theorem 6.23 (sketch). An antichain $\mathfrak{I}$ of proper intervals of $[n]$ is a collection of intervals $[i, j]=\{i, i+1, \ldots, j\}$ with $1 \leq i<j \leq n$ such that if 


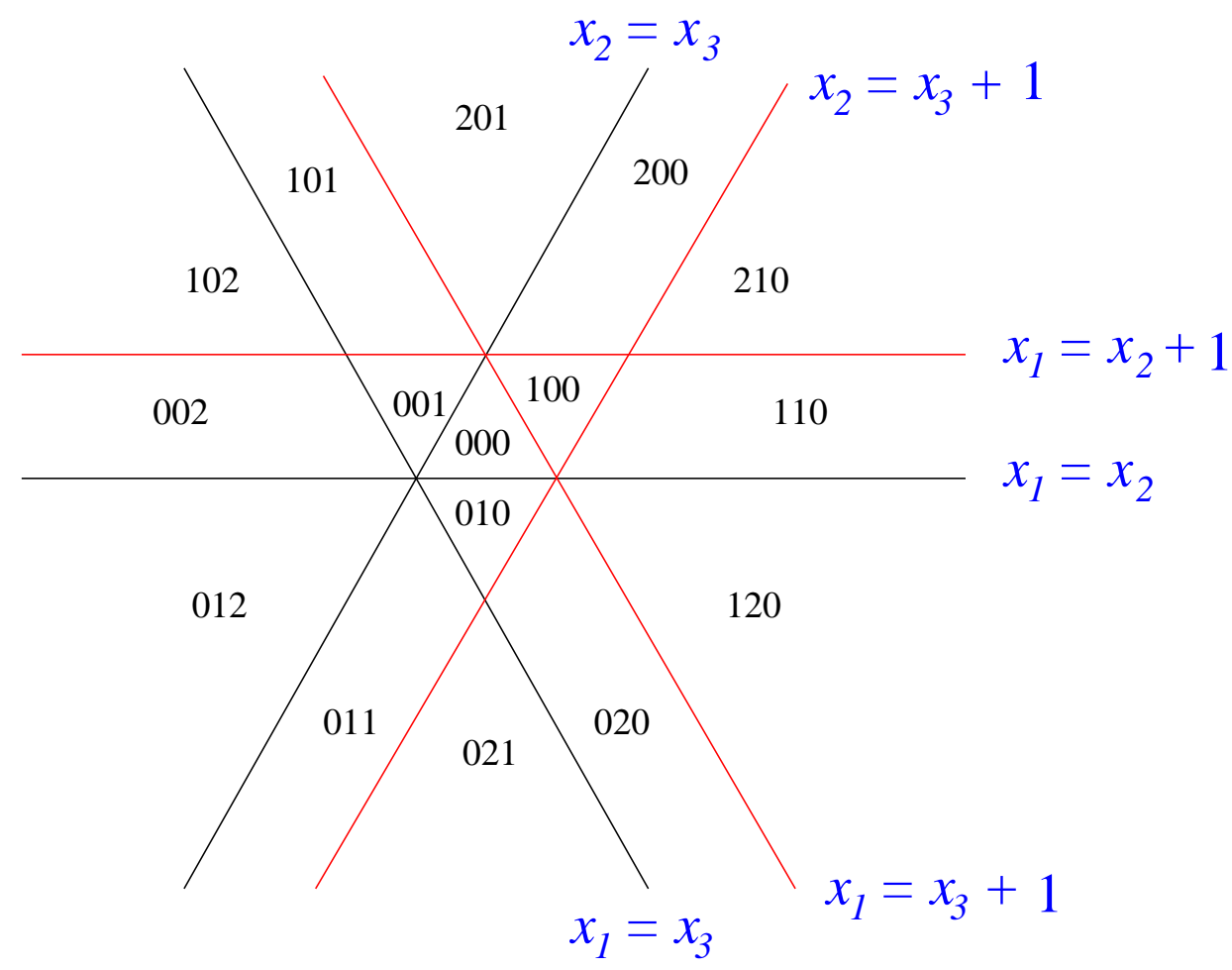

Figure 5. The labeling $\lambda$ of the regions of $\mathcal{S}_{3}$

$I, I^{\prime} \in \mathfrak{I}$ and $I \subseteq I^{\prime}$, then $I=I^{\prime}$. For instance, there are five antichains of proper intervals of [3], namely (writing $i j$ for $[i, j]$ )

$$
\emptyset,\{12\},\{23\},\{12,23\}, \quad\{13\} .
$$

In general, the number of antichains of proper intervals of $[n]$ is the Catalan number $C_{n}$ (immediate from [32, Exer. 6.19(bbb]), though this fact is not relevant here.

Every region $R \in \mathcal{R}\left(\mathcal{S}_{n}\right)$ corresponds bijectively to a pair $(w, \mathfrak{I})$, where $w \in \mathfrak{S}_{n}$ and $\mathfrak{I}$ is an antichain of proper intervals such that if $[i, j] \in \mathfrak{J}$ then $w(i)<w(j)$. Namely, the pair $(w, \mathfrak{I})$ corresponds to the region

$$
\begin{gathered}
x_{w(1)}>x_{w(2)}>\cdots>x_{w(n)} \\
x_{w(r)}-x_{w(s)}<1 \text { if }[r, s] \in \mathfrak{I}
\end{gathered}
$$

$x_{w(r)}-x_{w(s)}>1$ if $r<s, w(r)<w(s)$, and $\nexists[i, j] \in \mathfrak{I}$ such that $i \leq r<s \leq j$. We call $(w, \mathfrak{I})$ a valid pair. Given a valid pair $(w, \mathfrak{I})$ corresponding to a region $R$, write $d(w, \mathfrak{I})=d\left(R_{0}, R\right)$. It is easy to see that

$$
\begin{gathered}
d(w, \mathfrak{I})=\#\{(i, j): i<j, w(i)>w(j)\} \\
+\#\{(i, j): i<j, w(i)<w(j), \text { no } I \in \mathfrak{I} \text { satisfies } i, j \in I\} .
\end{gathered}
$$

We say that the pair $(i, j)$ is of type 1 if $i<j$ and $w(i)>w(j)$, and is of type 2 if $i<j, w(i)<w(j)$, and no $I \in \mathfrak{I}$ satisfies $i, j \in I$. Thus $d(w, \mathfrak{I})$ is the number of pairs $(i, j)$ that are either of type 1 or type 2 .

Example. Let $w=521769348$ and $\mathfrak{I}=\{14,27,49\}$. We can represent the pair $(w, \mathfrak{I})$ by the diagram 


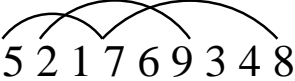

This corresponds to the region

$$
\begin{gathered}
x_{5}>x_{2}>x_{1}>x_{7}>x_{6}>x_{9}>x_{3}>x_{4}>x_{8} \\
x_{5}-x_{7}<1, x_{2}-x_{3}<1, x_{7}-x_{8}<1 .
\end{gathered}
$$

This region is separated from $R_{0}$ by the hyperplanes

$$
\begin{gathered}
x_{5}=x_{2}, x_{5}=x_{1}, \ldots(13 \text { in all }) \\
x_{5}=x_{6}+1, x_{5}=x_{9}+1, \ldots(7 \text { in all }) .
\end{gathered}
$$

Let $\lambda(w, \mathfrak{I}, w(i))$ be the number of integers $j$ such that $(i, j)$ is either of type 1 or type 2 . Thus

$$
\lambda(R)=(\lambda(w, \Im, 1), \ldots, \lambda(w, \mathfrak{I}, n)) .
$$

For the example above we have $\lambda(R)=(2,3,0,0,7,2,3,0,3)$. For instance, the entry $\lambda(w, \Im, 5)=7$ corresponds to the seven pairs $12,13,17,18$ (type 1 ) and 15 , 16, 19 (type 2).

Clearly $\lambda(R)+(1,1, \ldots, 1) \in \mathrm{PF}_{n}$, since $\lambda(w, \mathfrak{I}, w(i)) \leq n-i$ (the number of elements to the right of $w(i)$ in $w)$.

Key lemma. Let $X$ be an $r$-element subset of $[n]$, and let $v=v_{1} \cdots v_{r}$ be a permutation of $X$. Let $\mathfrak{J}$ be an antichain of proper intervals $[a, b]$, where $v_{a}<v_{b}$. Suppose that the pair $(i, j)$ is either of type 1 or type 2 . Then

$$
\lambda\left(v, \mathfrak{J}, v_{i}\right)>\lambda\left(v, \mathfrak{J}, v_{j}\right) .
$$

The proof of this lemma is straightforward and is left to the reader. For the example above, writing $\lambda(R)=\left(\lambda_{1}, \ldots, \lambda_{9}\right)=(5,2,1,7,6,9,3,4,8)$, the above lemma implies that

(a) $\lambda_{5}>\lambda_{2}, \lambda_{5}>\lambda_{1}, \lambda_{5}>\lambda_{3}, \lambda_{5}>\lambda_{4}, \lambda_{2}>\lambda_{1}, \lambda_{7}>\lambda_{6}, \lambda_{7}>\lambda_{3}, \lambda_{7}>\lambda_{4}$, $\lambda_{6}>\lambda_{3}, \lambda_{6}>\lambda_{4}, \lambda_{9}>\lambda_{3}, \lambda_{9}>\lambda_{4}, \lambda_{9}>\lambda_{8}$

(b) $\lambda_{5}>\lambda_{6}, \lambda_{5}>\lambda_{9}, \lambda_{5}>\lambda_{8}, \lambda_{2}>\lambda_{4}, \lambda_{2}>\lambda_{8}, \lambda_{1}>\lambda_{4}, \lambda_{1}>\lambda_{8}$.

The crux of the proof of Theorem 6.23 is to show that given $\alpha+(1,1, \ldots, 1) \in$ $\mathrm{PF}_{n}$, there is a unique region $R \in \mathcal{R}\left(\mathcal{S}_{n}\right)$ satisfying $\lambda(R)=\alpha$. We will illustrate the construction of $R$ from $\alpha$ with the example $\alpha=(2,3,0,0,7,2,3,0,3)$. We build up the pair $(w, \mathfrak{I})$ representing $R$ one step at a time. First let $v$ be the permutation of $[n]$ obtained from "standardizing" $\alpha$ from right-to-left. This means replacing the 0 's in $\alpha$ with $1,2, \ldots, m_{1}$ from right-to-left, then replacing the 1 's in $\alpha$ with $m_{1}+1, m_{1}+2, \ldots, m_{2}$ from right-to-left, etc. Let $v^{-1}=\left(t_{1}, \ldots, t_{n}\right)$. For our example, we have

$\begin{array}{ccccccccccc}\alpha & = & 2 & 3 & 0 & 0 & 7 & 2 & 3 & 0 & 3 \\ v & = & 5 & 8 & 3 & 2 & 9 & 4 & 7 & 1 & 6 \\ v^{-1} & = & 8 & 4 & 3 & 6 & 1 & 9 & 7 & 2 & 5\end{array}$.

Next we insert $t_{1}, \ldots, t_{n}$ from left-to-right into $w$. From $\alpha$ we can read off where $t_{i}$ is inserted. After inserting $t_{i}$, we also record which of the positions of the elements so far inserted belong to some interval $I \in \mathfrak{I}$. We can also determine from $\alpha$ the unique way to do this. The best way to understand this insertion technique is to practice with some examples. Figure 6 illustrates the steps in the insertion process for our current example. These steps are explained as follows. 
Figure 6. Constructing a valid pair $(w, \mathfrak{I})$ from the parking function $\alpha=(2,3,0,0,7,2,3,0,3)$

(1) First insert 8.

(2) Insert 4 . Since $\alpha_{8}=0,4$ appears to the left of 8 , so we have the partial permutation 48. We now must decide whether the positions of 4 and 8 belong to some interval $I \in \mathfrak{I}$. (In other words, in the pictorial representation of $(w, \mathfrak{I})$, will 4 and 8 lie under some arc?) By the first term on the right-hand side of (56), we would have $\alpha_{4} \geq 1$ if there were no such $I$. Since $\alpha_{4}=0$, we obtain the second row of Figure 6 .

(3) Insert 3. As in the previous step, we obtain 348 with a single arc over all three terms.

(4) Insert 6. Suppose we inserted it after the 3 , obtaining 3648 , with a single arc over all four terms (since 3 and 8 have already been determined to lie under a single arc). We have $\alpha_{6}=2$, but the contribution so far (of 3648 with an arc over all four terms) to $\alpha_{6}$ is 1 . Thus later we must insert some $j$ to the right of 6 so that the pair $(6, j)$ is of type 1 or type 2. By the lemma, we would have $\lambda(w, \mathfrak{I}, 6)>\lambda(w, \mathfrak{I}, j)$, contradicting that we are inserting elements in order of increasing $\alpha_{i}$ 's. Similarly 3468 and 3486 are excluded, so 6 must be inserted at the left, yielding 6348 . If the arc over $4,6,8$ is not extended to 6 , then we would have $\alpha_{6} \geq 3$. Hence we obtain the fourth row of Figure 6. 
(5) Insert 1. Using the lemma we obtain 16348. Since $\alpha_{1}=2$, there is an arc over 1 and two other elements to the right to 1 . This gives the fifth row of Figure 6.

(6) Insert 9. Placing 9 before 1 or 6 yields $\alpha_{9} \geq 4$, contradicting $\alpha_{9}=3$. Placing 9 after 3,4, or 8 is excluded by the lemma. Hence we get the sixth row of Figure 6.

(7) Insert 7. Placing 7 at the beginning yields four terms $j<7$ appearing to the right of 7 , giving $\alpha_{7} \geq 4$, a contradiction. Placing 7 after $6,9,3,4,8$ will violate the lemma, so we get the partial permutation 1769348. In order that $\alpha_{7}=3$, we must have 7 and 8 appearing under the same arc. Hence the arc from 6 to 8 must be extended to 7 , yielding row seven of Figure 6 .

(8) Insert 2 and 5. By now we hope it is clear that there is always a unique way to proceed.

The uniqueness of the above procedure shows that the map from the regions $R$ of $\mathcal{S}_{n}$ (or the valid pairs $(w, \mathfrak{I}$ ) that index the regions) to parking functions $\alpha$ is injective. Since the number of valid pairs and number of parking functions are both $(n+1)^{n-1}$, the map is bijective, completing the (sketched) proof. In fact, it's not hard to show surjectivity directly, i.e., that the above procedure produces a valid pair $(w, \mathfrak{I})$ for any parking function, circumventing the need to know that $r\left(\mathcal{S}_{n}\right)=\# \mathrm{PF}_{n}$ in advance.

Corollary 6.14. The distance enumerator of $\mathcal{S}_{n}$ is given by

$$
D_{\mathcal{S}_{n}}(t)=\sum_{\left(a_{1}, \ldots, a_{n}\right) \in \mathrm{PF}_{n}} t^{a_{1}+\cdots+a_{n}-n} .
$$

Proof. It is immediate from the definition of the labeling $\lambda: \mathcal{R}\left(\mathcal{S}_{n}\right) \rightarrow \mathbb{N}^{n}$ that if $\lambda(R)=\left(a_{1}, \ldots, a_{n}\right)$, then $d\left(R_{0}, R\right)=a_{1}+\cdots+a_{n}$. Now use Theorem 6.23.

Note. An alternative proof of Corollary 6.14 is given by Athanasiadis [3].

\subsection{The distance enumerator of a supersolvable arrangement}

The goal of this section is a formula for the distance enumerator of a supersolvable (central) arrangement with respect to a "canonical" base region $R_{0}$. The proof will be by induction, based on the following lemma of Björner, Edelman, and Ziegler $[8]$.

Lemma 6.7. Every central arrangement of rank 2 is supersolvable. A central arrangement $\mathcal{A}$ of rank $d \geq 3$ is supersolvable if and only if $\mathcal{A}=\mathcal{A}_{0} \bullet \mathcal{A}_{1}$ (disjoint union), where $\mathcal{A}_{0}$ is supersolvable of rank $d-1$ (so $\mathcal{A}_{1} \neq \emptyset$ ) and for all $H^{\prime}, H^{\prime \prime} \in \mathcal{A}_{1}$ with $H^{\prime} \neq H^{\prime \prime}$, there exists $H \in \mathcal{A}_{0}$ such that $H^{\prime} \cap H^{\prime \prime} \subseteq H$.

Proof. Every geometric lattice of rank 2 is modular, hence supersolvable, so let $\mathcal{A}$ be supersolvable of rank $d \geq 3$. Let $\hat{0}=x_{0} \lessdot x_{1} \lessdot \cdots \lessdot x_{d-1} \lessdot x_{d}=\hat{1}$ be a modular maximal chain in $L_{\mathcal{A}}$. Define

$$
\mathcal{A}_{0}=\mathcal{A}_{x_{d-1}}=\left\{H \in \mathcal{A}: x_{d-1} \subseteq H\right\},
$$

so $L\left(\mathcal{A}_{0}\right) \cong\left[\hat{0}, x_{d+1}\right]$. Clearly $\mathcal{A}_{0}$ is supersolvable of rank $d-1$. Let $\mathcal{A}_{1}=\mathcal{A}-\mathcal{A}_{0}$. Let $H^{\prime}, H^{\prime \prime} \in \mathcal{A}_{1}, H^{\prime} \neq H^{\prime \prime}$. Since $x_{d-1} \nsubseteq H^{\prime}$ we have $x_{d-1} \vee\left(H^{\prime} \vee H^{\prime \prime}\right)=\hat{1}$ in $L(\mathcal{A})$. Now $\operatorname{rk}\left(x_{d-1}\right)=d-1$, and $\operatorname{rk}\left(H^{\prime} \vee H^{\prime \prime}\right)=2$ by semimodularity. Since $x_{d-1}$ is modular we obtain

$$
\operatorname{rk}\left(x_{d-1} \wedge\left(H^{\prime} \vee H^{\prime \prime}\right)\right)=(d-1)+2-d=1
$$


i.e., $x_{d-1} \wedge\left(H^{\prime} \vee H^{\prime \prime}\right)=H \in \mathcal{A}$. Since $H \leq x_{d-1}$ it follows that $H \in \mathcal{A}_{0}$. Moreover, $H^{\prime} \cap H^{\prime \prime} \subseteq H$ since $H \leq H^{\prime} \vee H^{\prime \prime}$. This proves the "only if" part of the lemma. The "if" part is straightforward and not needed here, so we omit the proof.

Given $\mathcal{A}_{0}=\mathcal{A}_{x_{d-1}}$ as above, define a map $\pi: \mathcal{R}(\mathcal{A}) \rightarrow \mathcal{R}\left(\mathcal{A}_{0}\right)$ (the symbol $\rightarrow$ denotes surjectivity) by $\pi(R)=R^{\prime}$ if $R \subseteq R^{\prime}$. For $R \in \mathcal{R}(\mathcal{A})$ let

$$
\mathcal{F}(R)=\left\{R_{1} \in \mathcal{R}(\mathcal{A}): \pi(R)=\pi\left(R_{1}\right)\right\}=\pi^{-1}(\pi(R)) .
$$

For example, let $\mathcal{A}$ be the arrangement

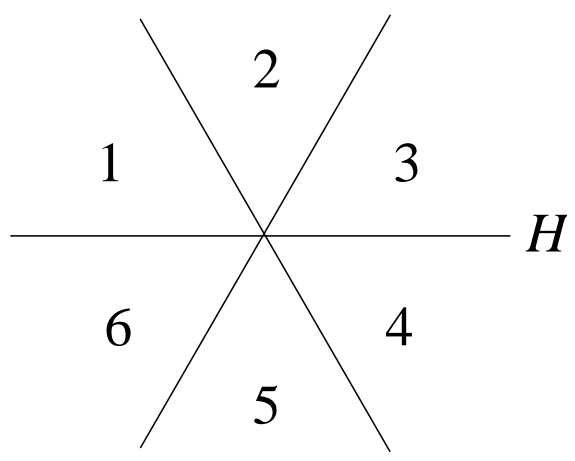

Let $\mathcal{A}_{0}=\{H\}$. Then $\mathcal{F}(1)=\{1,2,3\}$ and $\mathcal{F}(5)=\{4,5,6\}$.

Now let $R^{\prime} \in \mathcal{R}\left(\mathcal{A}_{0}\right)$. By Lemma 6.7 no $H^{\prime}, H^{\prime \prime} \in \mathcal{A}$ can intersect inside $R^{\prime}$. The illustration below is a projective diagram of a bad intersection. The solid lines define $\mathcal{A}_{0}$ and the dashed lines $\mathcal{A}_{1}$.

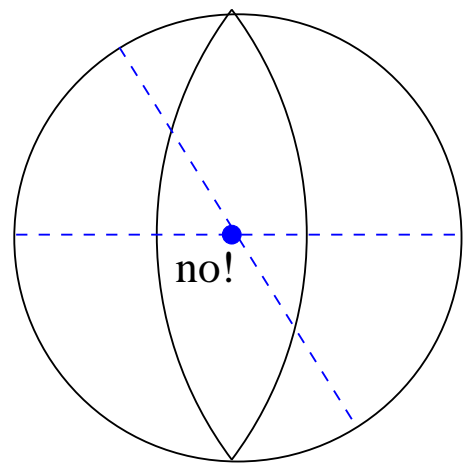

Thus $\pi^{-1}\left(R^{\prime}\right)$ must be arranged "linearly" in $R^{\prime}$, i.e., there is a straight line intersecting all $R \in \pi^{-1}\left(R^{\prime}\right)$. 


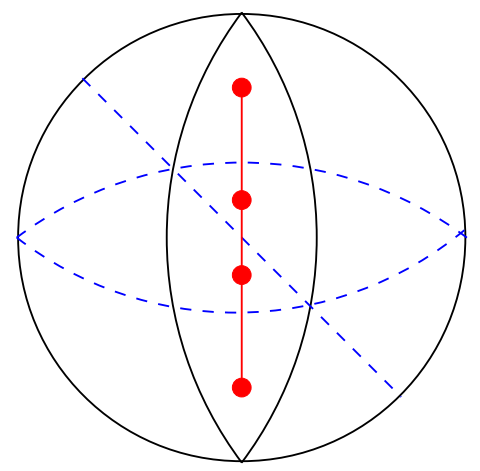

Since $\operatorname{rank}(\mathcal{A})>\operatorname{rank}\left(\mathcal{A}_{0}\right)$, we have $\# \pi^{-1}\left(R^{\prime}\right)>1$ (for $H \in \mathcal{A}$ does not bisect $R^{\prime}$ if and only if $\left.\operatorname{rank}\left(\mathcal{A}_{0} \cup H\right)=\operatorname{rank}\left(\mathcal{A}_{0}\right)\right)$. Thus there are two distinct regions $R_{1}, R_{2} \in \pi^{-1}\left(R^{\prime}\right)$ that are endpoints of the "chain of regions."

Let $e_{d}$ have the meaning of equation (34), i.e.,

$$
e_{d}=\#\left\{H \in \mathcal{A}: H \notin \mathcal{A}_{0}\right\}=\# \mathcal{A}_{1} \text {. }
$$

Then $\pi^{-1}\left(R^{\prime}\right)$ is a chain of regions of length $e_{d}$, so $\# \pi^{-1}\left(R^{\prime}\right)=1+e_{d}$. We now come to the key definition of this subsection. The definition is recursive by rank, the base case being rank at most 2 .

Definition 6.16. Let $\mathcal{A}$ be a real supersolvable central arrangement of rank $d$, and let $\mathcal{A}_{0}$ be a supersolvable subarrangement of $\operatorname{rank} d-1$ (which always exists by the definition of supersolvability). A region $R_{0} \in \mathcal{R}(\mathcal{A})$ is called canonical if either (1) $d \leq 2$, or else (2) $d \geq 3, \pi\left(R_{0}\right) \in \mathcal{R}\left(\mathcal{A}_{0}\right)$ is canonical, and $R_{0}$ is an endpoint of the chain $\mathcal{F}\left(R_{0}\right)$.

Since every chain has two endpoints and a central arrangement of rank 1 has two (canonical) regions, it follows that there are at least $2^{d}$ canonical regions.

The main result on distance enumerators of supersolvable arrangements is the following, due to Björner, Edelman, and Ziegler [8, Thm. 6.11].

Theorem 6.24. Let $\mathcal{A}$ be a supersolvable central arrangement of rank $d$ in $\mathbb{R}^{n}$. Let $R_{0} \in \mathcal{R}(\mathcal{A})$ be canonical, and suppose that

$$
\chi_{\mathcal{A}}(t)=\left(t-e_{1}\right)\left(t-e_{2}\right) \cdots\left(t-e_{d}\right) t^{n-d} .
$$

(There always exist such positive integers $e_{i}$ by Corollary 4.9.) Then

$$
D_{\mathcal{A}, R_{0}}(t)=\prod_{i=1}^{d}\left(1+t+t^{2}+\cdots+t^{e_{i}}\right) .
$$

Proof. Let $W_{\mathcal{A}}$ be the weak order on $\mathcal{A}$ with respect to $R_{0}$, i.e.,

$$
W_{\mathcal{A}}=\left\{\operatorname{sep}\left(R_{0}, R\right): R \in \mathcal{R}(\mathcal{A})\right\},
$$

ordered by inclusion. Thus $W_{\mathcal{A}}$ is graded with rank function given by $\operatorname{rk}(R)=$ $d\left(R_{0}, R\right)$ and rank generating function

$$
\sum_{R \in W_{\mathcal{A}}} t^{\mathrm{rk}(R)}=D_{\mathcal{A}}(t)
$$


Since $R_{0}$ is canonical, for all $R^{\prime} \in \mathcal{R}\left(\mathcal{A}_{0}\right)$ we have that $\pi^{-1}\left(R^{\prime}\right)$ is a chain of length $e_{d}$. Hence if $R \in \mathcal{R}(\mathcal{A})$ and $h(R)$ denotes the rank of $R$ in the chain $\mathcal{F}(R)$, then

$$
d_{\mathcal{A}}\left(R_{0}, R\right)=d_{\mathcal{A}_{0}}(\pi(R))+h(R) .
$$

Therefore

$$
D_{\mathcal{A}}(t)=D_{\mathcal{A}_{0}}(t)\left(1+t+\cdots+t^{e_{d}}\right),
$$

and the proof follows by induction.

NoTE. The following two results were also proved in [8]. We simply state them here without proof.

- If $\mathcal{A}$ is a real supersolvable central arrangement and $R_{0}$ is canonical, then $W_{\mathcal{A}}$ is a lattice (Exercise 7 ).

- If $\mathcal{A}$ is any real central arrangement and $W_{\mathcal{A}}$ is a lattice, then $R_{0}$ is simplicial (bounded by exactly $\operatorname{rk}(\mathcal{A})$ hyperplanes, the minimum possible). In other words, the closure $\bar{R}_{0}$ is a simplex. As a partial converse, if every region $R$ is simplicial, then $W_{\mathcal{A}}$ is a lattice (Exercise 8).

\subsection{The Varchenko matrix}

Let $\mathcal{A}$ be a real arrangement. For each $H \in \mathcal{A}$ let $a_{H}$ be an indeterminate. Define a matrix $V=V(\mathcal{A})$ with rows and columns indexed by $\mathcal{R}(\mathcal{A})$ by

$$
V_{R R^{\prime}}=\prod_{H \in \operatorname{sep}\left(R, R^{\prime}\right)} a_{H} .
$$

For instance, let $\mathcal{A}$ be given as follows:

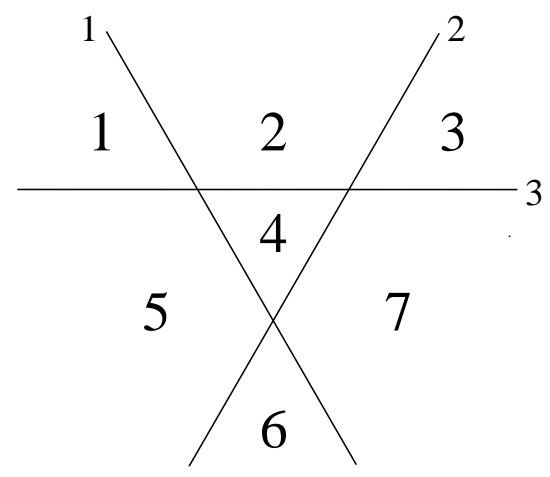

Then

\begin{tabular}{c|ccccccc} 
& 1 & 2 & 3 & 4 & 5 & 6 & 7 \\
\hline 1 & 1 & $a_{1}$ & $a_{1} a_{2}$ & $a_{1} a_{3}$ & $a_{3}$ & $a_{2} a_{3}$ & $a_{1} a_{2} a_{3}$ \\
2 & $a_{1}$ & 1 & $a_{2}$ & $a_{3}$ & $a_{1} a_{3}$ & $a_{1} a_{2} a_{3}$ & $a_{2} a_{3}$ \\
3 & $a_{1} a_{2}$ & $a_{2}$ & 1 & $a_{2} a_{3}$ & $a_{1} a_{2} a_{3}$ & $a_{1} a_{3}$ & $a_{3}$ \\
4 & $a_{1} a_{3}$ & $a_{3}$ & $a_{2} a_{3}$ & 1 & $a_{1}$ & $a_{1} a_{2}$ & $a_{2}$ \\
5 & $a_{3}$ & $a_{1} a_{3}$ & $a_{1} a_{2} a_{3}$ & $a_{1}$ & 1 & $a_{2}$ & $a_{1} a_{2}$ \\
6 & $a_{2} a_{3}$ & $a_{a} a_{2} a_{3}$ & $a_{1} a_{3}$ & $a_{1} a_{2}$ & $a_{2}$ & 1 & $a_{1}$ \\
7 & $a_{1} a_{2} a_{3}$ & $a_{1} a_{3}$ & $a_{3}$ & $a_{2}$ & $a_{1} a_{2}$ & $a_{1}$ & 1
\end{tabular}

The determinant of this matrix happens to be given by

$$
\operatorname{det}(V)=\left(1-a_{1}^{2}\right)^{3}\left(1-a_{2}^{2}\right)^{3}\left(1-a_{3}^{2}\right)^{3} .
$$


In order to state the general result, define for $x \in L(\mathcal{A})$,

$$
\begin{aligned}
a_{x} & =\prod_{H \supseteq x} a_{H} \\
n(x) & =r\left(\mathcal{A}^{x}\right) \\
p(x) & =b\left(c^{-1} \mathcal{A}_{x}\right)=\beta\left(\mathcal{A}_{x}\right),
\end{aligned}
$$

where as usual $\mathcal{A}^{x}=\{x \cap H \neq \emptyset: x \nsubseteq H\}$ and $\mathcal{A}_{x}=\{H \in \mathcal{A}: H \supseteq x\}$, and where $c^{-1}$ denotes deconing and $\beta$ is defined in Exercise 4.22. Thus

$$
\begin{gathered}
n(x)=\left|\chi_{\mathcal{A}^{x}}(-1)\right|=\sum_{y \geq x}|\mu(x, y)| \\
p(x)=\left|\chi_{\mathcal{A}_{x}}^{\prime}(1)\right| .
\end{gathered}
$$

Example 6.14. The arrangement of three lines illustrated above has two types of intersections (other than 0): a line $x$ and a point $y$. For a line $x, \mathcal{A}^{x}$ consists of two points on a line, so $n(x)=r\left(\mathcal{A}^{x}\right)=3$. Moreover, $\mathcal{A}_{x}$ consists of the single hyperplane $x$ in $\mathbb{R}^{2}$, so $c^{-1} \mathcal{A}_{x}=\emptyset$ and $p(x)=b(\emptyset)=1$. Hence we obtain the factor $\left(1-a_{x}\right)^{3}$ in the determinant. On the other hand, $\mathcal{A}^{y}=\emptyset$ so $n(y)=r(\emptyset)=1$. Moreover, $\mathcal{A}_{y}$ consists of two intersecting lines in $\mathbb{R}^{2}$, with characteristic polynomial $\chi_{\mathcal{A}_{y}}(t)=(t-1)^{2}$. Hence $p(y)=\left|\chi_{\mathcal{A}_{y}}^{\prime}(1)\right|=0$. Equivalently, $c^{-1} \mathcal{A}_{y}$ consists of a single point on a line, so again $p(y)=b\left(c^{-1} \mathcal{A}_{y}\right)=0$. Thus $y$ contributes a factor $\left(1-a_{y}^{2}\right)^{0}=1$ to $\operatorname{det}(V)$.

We can now state the remarkable result of Varchenko [37], generalized to "weighted matroids" by Brylawski and Varchenko [11].

Theorem 6.25. Let $\mathcal{A}$ be a real arrangement. Then

$$
\operatorname{det} V(\mathcal{A})=\prod_{\hat{0} \neq x \in L(\mathcal{A})}\left(1-a_{x}^{2}\right)^{n(x) p(x)} .
$$

Proof. Omitted. 


\section{Exercises}

(1) Let $\mathcal{A}$ be a central arrangement in $\mathbb{R}^{n}$ with distance enumerator $D_{\mathcal{A}}(t)$ (with respect to some base region $R_{0}$ ). Define a graph $G_{\mathcal{A}}$ on the vertex set $\mathcal{R}(\mathcal{A})$ by putting an edge between $R$ and $R^{\prime}$ if $\# \operatorname{sep}\left(R, R^{\prime}\right)=1$ (i.e., $R$ and $R^{\prime}$ are separated by a unique hyperplane).

(a) [2-] Show that $G_{\mathcal{A}}$ is a bipartite graph.

(b) [2] Show that if $\# \mathcal{A}$ is odd, then $D_{\mathcal{A}}(-1)=0$.

(c) [2] Show that if $\# \mathcal{A}$ is even and $r(\mathcal{A}) \equiv 2(\bmod 4)$, then $D_{\mathcal{A}}(-1) \equiv 2(\bmod 4)$ (so $D_{\mathcal{A}}(-1) \neq 0$ ).

(d) [2] Give an example of (c), i.e., find $\mathcal{A}$ so that $\# \mathcal{A}$ is even and $r(\mathcal{A}) \equiv$ $2(\bmod 4)$.

(e) [2] Show that (c) cannot hold if $\mathcal{A}$ is supersolvable. (It is not assumed that the base region $R_{0}$ is canonical. Try to avoid the use of Section 6.4.)

(f) $[2+]$ Show that if $\# \mathcal{A}$ is even and $r(\mathcal{A}) \equiv 0(\bmod 4)$, then it is possible for $D_{\mathcal{A}}(-1)=0$ and for $D_{\mathcal{A}}(-1) \neq 0$. Can examples be found for $\operatorname{rank}(\mathcal{A}) \leq 3$ ?

(2) [2-] Show that a sequence $\left(c_{1}, \ldots, c_{n}\right) \in \mathbb{N}^{n}$ is the inversion sequence of a permutation $w \in \mathfrak{S}_{n}$ if and only if $c_{i} \leq i-1$ for $1 \leq i \leq n$.

(3) [2] Show that all cars can park under the scenario following Definition 6.15 if and only if the sequence $\left(a_{1}, \ldots, a_{n}\right)$ of preferred parking spaces is a parking function.

(4) [5] Find a bijective proof of Theorem 6.22, i.e., find a bijection $\varphi$ between the set of all rooted forests on $[n]$ and the set $\mathrm{PF}_{n}$ of all parking functions of length $n$ satisfying $\operatorname{inv}(F)=\left(\begin{array}{c}n+1 \\ 2\end{array}\right)-a_{1}-\cdots-a_{n}$ when $\varphi(F)=\left(a_{1}, \ldots, a_{n}\right)$. Note. In principle a bijection $\varphi$ can be obtained by carefully analyzing the proof of Theorem 6.22. However, this bijection will be of a messy recursive nature. A "nonrecursive" bijection would be greatly preferred.

(5) [5] There is a natural two-variable refinement of the distance enumerator (57) of $\mathcal{S}_{n}$. Given $R \in \mathcal{R}\left(\mathcal{S}_{n}\right)$, define $d_{0}\left(R_{0}, R\right)$ to be the number of hyperplanes $x_{i}=x_{j}$ separating $R_{0}$ from $R$, and $d_{1}\left(R_{0}, R\right)$ to be the number of hyperplanes $x_{i}=x_{j}+1$ separating $R_{0}$ from $R$. (Here $R_{0}$ is given by (55) as usual.) Set

$$
D_{n}(q, t)=\sum_{R \in \mathcal{R}\left(\mathcal{S}_{n}\right)} q^{d_{0}\left(R_{0}, R\right)} t^{d_{1}\left(R_{0}, R\right)} .
$$

What can be said about the polynomial $D_{n}(q, t)$ ? Can its coefficients be interpreted in a simple way in terms of tree or forest inversions? Are there formulas or recurrences for $D_{n}(q, t)$ generalizing Theorem 6.21 , Corollary 6.13 , or equation (53)? The table below give the coefficients of $q^{i} t^{j}$ in $D_{n}(q, t)$ for $2 \leq n \leq 4$.

\begin{tabular}{l|ll}
$t \backslash^{q}$ & 0 & 1 \\
\hline 0 & 1 & 1 \\
1 & 1 &
\end{tabular}

\begin{tabular}{l|llll}
$t \backslash^{q}$ & 0 & 1 & 2 & 3 \\
\hline 0 & 1 & 1 & 2 & 1 \\
1 & 2 & 2 & 2 & \\
2 & 2 & 2 & & \\
3 & 1 & & &
\end{tabular}

\begin{tabular}{l|lllllll}
$t \backslash^{q}$ & 0 & 1 & 2 & 3 & 4 & 5 & 6 \\
\hline 0 & 1 & 1 & 2 & 3 & 3 & 3 & 1 \\
1 & 3 & 3 & 6 & 7 & 6 & 3 & \\
2 & 5 & 5 & 8 & 9 & 5 & & \\
3 & 6 & 7 & 9 & 6 & & & \\
4 & 5 & 6 & 5 & & & & \\
5 & 3 & 3 & & & & & \\
6 & 1 & & & & & &
\end{tabular}


Some entries of these table are easy to understand, e.g., the first and last entries in each row and column, but a simple way to compute the entire table is not known.

(6) [5-] Let $\mathcal{G}_{n}$ denote the generic braid arrangement

$$
x_{i}-x_{j}=a_{i j}, \quad 1 \leq i<j \leq n,
$$

in $\mathbb{R}^{n}$. Can anything interesting be said about the distance enumerator $D_{\mathcal{G}_{n}}(t)$ (which depends on the choice of base region $R_{0}$ and possibly on the $a_{i j}$ 's)? Generalize if possible to generic graphical arrangments, especially for supersolvable (or chordal) graphs.

(7) [3-] Let $\mathcal{A}$ be a real supersolvable arrangement and $R_{0}$ a canonical region of $\mathcal{A}$. Show that the weak order $W_{\mathcal{A}}$ (with respect to $R_{0}$ ) is a lattice.

(8) (a) $[2+]$ let $\mathcal{A}$ be a real central arrangement of rank $d$. Suppose that the weak order $W_{\mathcal{A}}$ (with respect to some region $R_{0} \in \mathcal{R}(\mathcal{A})$ ) is a lattice. Show that $R_{0}$ is simplicial, i.e., bounded by exactly $d$ hyperplanes.

(b) [3-] Let $\mathcal{A}$ be a real central arrangement. Show that if every region $R \in$ $\mathcal{R}(\mathcal{A})$ is simplicial, then $W_{\mathcal{A}}$ is a lattice.

(9) (a) [2] Set each $a_{H}=q$ in the Varchenko matrix $V$ of an arrangement $R$ in $\mathbb{R}^{n}$, obtaining a matrix $V(q)$. Let $r=r(\mathcal{A})$. The entries of $V(q)$ belong to the principal ideal domain $\mathbb{Q}[q]$, so $V(q)$ has a Smith normal form $A V(q) B=$ $\operatorname{diag}\left(p_{1}, \ldots, p_{r}\right)$, where $A, B$ are $r \times r$ matrices whose entries belong to $\mathbb{Q}[q]$ and whose determinants are nonzero elements of $\mathbb{Q}$, and where $p_{1}, \ldots, p_{r} \in$ $\mathbb{Q}[q]$ such that $p_{i} \mid p_{i+1}$ for $1 \leq i \leq r-1$. The Smith normal form is unique up to multiplication of the $p_{i}$ 's by nonzero elements of $\mathbb{Q}$. For instance, if $\mathcal{A}=\mathcal{B}_{3}$, then

$$
A V(q) B=\operatorname{diag}\left(1, q^{2}-1, q^{2}-1, q^{2}-1,\left(q^{2}-1\right)^{2},\left(q^{2}-1\right)^{2}\left(q^{4}+q^{2}+1\right)\right) .
$$

Show that each $p_{i}$ is a polynomial in $q^{2}$.

(b) $[3+]$ Let $a_{i}$ be the number of $j$ 's for which $\left(q^{2}-1\right)^{i} \mid p_{j}$ but $\left(q^{2}-1\right)^{i+1} \nmid p_{j}$. Show that

$$
\chi_{\mathcal{A}}(t)=\sum_{i \geq 0}(-1)^{n-i} a_{i} q^{n-i} .
$$

(c) [5] What more can be said about the polynomials $p_{i}$ ? By Theorem 6.25 they are products of cyclotomic polynomials, so one could begin by asking for the largest powers of $q^{2}+1$ or $q^{4}+q^{2}+1$ dividing each $p_{i}$. 



\section{BIBLIOGRAPHY}

1. C. A. Athanasiadis, Algebraic Combinatorics of Graph Spectra, Subspace Arrangements and Tutte Polynomials, Ph.D. thesis, MIT, 1996.

2. C. A. Athanasiadis, Characteristic polynomials of subspace arrangements and finite fields, Advances in Math. 122 (1996), 193-233.

3. C. A. Athanasiadis, A class of labeled posets and the Shi arrangement of hyperplanes, J. Combinatorial Theory (A) 80 (1997), 158-162.

4. H. Barcelo and E. Ihrig, Lattices of parabolic subgroups in connection with hyperplane arrangements, J. Algebraic Combinatorics 9 (1999), 5-24.

5. A. Björner, Orderings of Coxeter groups, in Combinatorics and algebra (Boulder, Colo., 1983), Contemp. Math. 34, American Mathematical Society, Providence, RI, 1984, pp. 175-195.

6. A. Björner and F. Brenti, Combinatorics of Coxeter Groups, to appear.

7. A. Björner, M. Las Vergnas, B. Sturmfels, N. White, and G. Ziegler, Oriented Matroids, second ed., Encyclopedia of Mathematics and Its Applications 46, Cambridge University Press, Cambridge, 1999.

8. A. Björner, P. Edelman, and G. Ziegler, Hyperplane arrangements with a lattice of regions, Discrete Comput. Geom. 5 (1990), 263-288.

9. A. Blass and B. Sagan, Characteristic and Ehrhart polynomials, J. Algebraic Combinatorics 7 (1998), 115-126.

10. N. Bourbaki, Groupes et algèbres de Lie, Éléments de Mathématique, Fasc. XXXIV, Hermann, Paris, 1968.

11. T. Brylawski and A. Varchenko, The determinant formula for a matroid bilinear form, Advances in Math. 129 (1997), 1-24.

12. J. L. Chandon, J. Lemaire, and J. Pouget, Dénombrement des quasi-ordres sur un ensemble fini, Math. Inform. Sci. Humaines 62 (1978), 61-80, 83.

13. H. Crapo and G.-C. Rota, On the Foundations of Combinatorial Theory: Combinatorial Geometries, preliminary edition, MIT Press, Cambridge, MA, 1970.

14. P. Edelman and C. Greene, Balanced tableaux, Advances in Math. 63 (1987), 42-99.

15. P. C. Fishburn, Interval Orders and Interval Graphs, Wiley-Interscience, New York, 1985.

16. D. Foata and J. Riordan, Mappings of acyclic and parking functions, Aequationes Math. 10 (1974), 10-22. 
17. A. Garsia, The saga of reduced factorizations of elements of the symmetric group, Publ. LACIM 29, Univerité du Québec à Montréal, 2002.

18. I. Gessel and D. L. Wang, Depth-first search as a combinatorial correspondence, J. Combinatorial Theory (A) 26 (1979), 308-313.

19. C. Greene, On the Möbius algebra of a partially ordered set, Advances in Math. 10 (1973), 177-187.

20. J. E. Humphreys, Reflection Groups and Coxeter Groups, Cambridge University Press, Cambridge, 1990.

21. A. G. Konheim and B. Weiss, An occupancy discipline and applications, SIAM J. Applied Math. 14 (1966), 1266-1274.

22. G. Kreweras, Une famille de polynômes ayant plusieurs propriétés énumerative, Per. Math. Hung. 11 (1980), 309-320.

23. C. L. Mallows and J. Riordan, The inversion enumerator for labeled trees, Bull. Amer. Math. Soc. 74, (1968), 92-94.

24. P. Orlik and H. Terao, Arrangements of Hyperplanes, Springer-Verlag, Berlin, 1992.

25. A. Postnikov and R. Stanley, Deformations of Coxeter hyperplane arrangements, J. Combinatorial Theory (A) 91 (2000), 544-597.

26. J. Riordan, Ballots and trees, J. Combinatorial Theory 6 (1969), 408-411

27. J.-Y. Shi, The Kazhdan-Lusztig cells in certain affine Weyl groups, Lecture Notes in Mathematics, vol. 1179, Springer-Verlag, Berlin, 1986.

28. R. Stanley, Modular elements of geometric lattices, Algebra Universalis 1 (1971), 214-217.

29. R. Stanley, Supersolvable lattices, Algebra Universalis 2 (1972), 197-217.

30. R. Stanley, On the number of reduced decompositions of elements of Coxeter groups, European J. Combinatorics 5 (1984), 359-372.

31. R. Stanley, Enumerative Combinatorics, vol. 1, Wadsworth and Brooks/Cole, Pacific Grove, CA, 1986; second printing, Cambridge University Press, Cambridge, 1996.

32. R. Stanley, Enumerative Combinatorics, vol. 2, Cambridge University Press, Cambridge, 1999

33. R. Stanley, Hyperplane arrangements, interval orders, and trees, Proc. Nat. Acad. Sci. 93 (1996), 2620-2625.

34. H. Terao, Free arrangements of hyperplanes and unitary reflection groups, Proc. Japan Acac., Ser. A 56 (1980), 389-392.

35. H. Terao, Generalized exponents of a free arrangement of hyperplanes and Shepherd[sic]-Todd-Brieskorn formula, Invent. math. 63 (1981), 159-179.

36. W. T. Trotter, Combinatorics and Partially Ordered Sets, The Johns Hopkins Univ. Press, Baltimore/London, 1992.

37. A. Varchenko, Bilinear form of real configuration of hyperplanes, Advances in Math. 97 (1993), 110-144.

38. R. L. Wine and J. E. Freund, On the enumeration of decision patterns involving $n$ means, Ann. Math. Stat. 28, 256-259. 